\title{
Validation of the Bookend Method in Dynamic Contrast Enhanced MRI
}

by

Brandon Zanette

\author{
A thesis submitted to \\ the Faculty of Graduate Studies and Research \\ in partial fulfilment of \\ the requirements for the degree of \\ Master of Science
}

in

Physics

Carleton University

Ottawa, Ontario, Canada

July 2013

Copyright (C)

2013 - Brandon Zanette 


\section{Abstract}

The quantitative accuracy of contrast agent concentration measurements for use in Dynamic Contrast Enhanced MRI is investigated in phantom experiments. Two commonly accepted techniques are compared. Both techniques require the direct input of $T_{1}$ measurements. Three candidates for clinical $T_{1}$ mapping with different overall accuracies were used to yield a total of six possible concentration evaluation methods. Measurements of concentration using these methods were repeated several times such that the overall accuracy and reproducibility may be evaluated. 


\section{Acknowledgments}

Firstly, I would like to thank my family, especially my parents, without whose support and motivation, this work would not be possible. Next, I would like to thank all of my friends, both old and new, for the many good times shared and the often needed reprieve that was provided during the completion of this work. I would also like to thank any and all of the people at Carleton University and The Ottawa Hospital who offered me a helping hand, academically or otherwise. Finally and without question, most importantly, I would like to thank Dr. Greg Cron and my supervisor, Dr. Ian Cameron. Without their help and patience, this work would not exist. They have helped me to grow as a student, while treating me as a colleague. Their passion for their work and their enthusiastic mentorship have opened the door to the exciting and fascinating world of MRI. I am grateful that I had the good fortune to benefit from their knowledge and expertise. As scientists, I am proud to have worked with them, however I am even more proud to call them my friends. 


\section{Table of Contents}

Abstract $\quad$ ii

Acknowledgments $\quad$ iii

Table of Contents $\quad$ iv

List of Tables $\quad$ vii

List of Figures $\quad$ x

List of Acronyms xiv

List of Symbols $\quad$ xvi

1 Introduction 1

2 Theory and Background 5

2.1 Basic MR Theory . . . . . . . . . . . . . . . 5

2.1.1 Classical Description of Nuclear Magnetic Resonance . . . . . 5

2.1.2 Origins of the MR Signal . . . . . . . . . . . . . . 7

2.1.3 Behaviour of $\boldsymbol{M}$, the Rotating Frame, and $\boldsymbol{B}_{\mathbf{1}} \ldots \ldots$

2.1.4 Bloch Equations and Relaxation . . . . . . . . . . . . . 12

2.2 Dynamic Contrast Enhanced MRI . . . . . . . . . . . . 15

2.2.1 Introduction to DCE-MRI . . . . . . . . . . . . . . . 15 
2.2 .2 Fundamentals of DCE-MRI . . . . . . . . . . . . . 16

2.2.3 Vascular Input and Kinetic Modelling . . . . . . . . . . . . 18

2.3 Concentration Quantification of Gadolinium-based Contrast Agent . . 22

2.3.1 DCE Signal Acquisition . . . . . . . . . . . . . . . 22

2.3.2 The Conventional $T_{1 \text { pre }}$ Method . . . . . . . . . . . . 23

2.3.3 The Bookend Method . . . . . . . . . . . . . . . 24

2.3.4 Justification for the Use of the Bookend Method . . . . . . . 25

3 Materials and Methods $\quad 29$

3.1 The Phantom . . . . . . . . . . . . . . . . . . . . . . . . . . . . . . . . . 29

3.2 DCE Signal Acquisition . . . . . . . . . . . . . . . . . 31

$3.3 T_{1}$ Quantification . . . . . . . . . . . . . . . 35

3.3.1 Gold Standard: Traditional Inversion Recovery _. . . . . . 36

3.3.2 The Variable Flip Angle Method _. . . . . . . . . . . . 38

3.3.3 Modified Look-Locker Inversion Recovery . . . . . . . . . . . 42

3.4 Phantom Experiments . . . . . . . . . . . . . . . . . . 45

3.4.1 Measurements . . . . . . . . . . . . . . . . . 45

3.4 .2 Postprocessing and Image Analysis _ . . . . . . . . . . 47

4 Results $\quad 50$

4.1 Reference Values . . . . . . . . . . . . . . . . . . 50

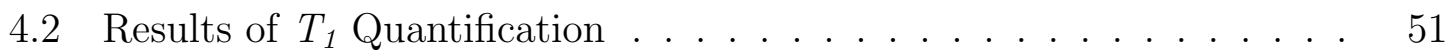

4.2.1 Variable Flip Angle Results _. . . . . . . . . . . . . 51

$4.2 .2 \quad$ MOLLI Results . . . . . . . . . . . . . . 56

4.3 Results of $[G d]$ Quantification $\ldots \ldots \ldots \ldots \ldots \ldots$

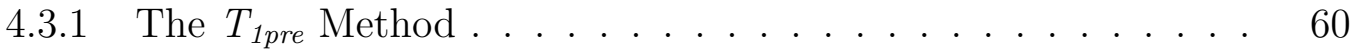

4.3.2 The Bookend Method _. . . . . . . . . . . . 67

4.3 .3 Interslice Variations . . . . . . . . . . . . . . . . . . . . 74 
5 Analysis and Discussion $\quad 78$

$5.1 T_{1}$ Quantification and Accuracy . . . . . . . . . . . 78

5.2 Accuracy, Precision, Spread, and Interslice Variation of [Gd] Results . 81

5.2.1 Effect on Accuracy . . . . . . . . . . . . . . . . . . . 82

5.2.2 Effect on Precision . . . . . . . . . . . . . 86

5.2.3 Effect on Spread . . . . . . . . . . . . . . . 89

5.2 .4 Overall Effect . . . . . . . . . . . . . . . . . . . 94

5.2.5 Through Plane Variation . . . . . . . . . . . . . . . 95

6 Conclusion $\quad 102$

6.1 Final Comments: The Bookend Method versus The $T_{1 \text { pre }}$ Method . . 102

6.2 Limitations of this work . . . . . . . . . . . . . . . 104

6.3 Future Work . . . . . . . . . . . . . . . . . . 105

$\begin{array}{ll}\text { List of References } & 107\end{array}$ 


\section{List of Tables}

3.1 Clinical 3D-FLASH pulse sequence parameters. . . . . . . . . . 35

3.2 TSE-IR pulse sequence parameters. . . . . . . . . . . . . . . 38

3.3 VFA pulse sequence parameters. . . . . . . . . . . . . . . 42

3.4 MOLLI pulse sequence parameters. . . . . . . . . . . . 45

4.1 Table showing results of the $\mathrm{uVFA} T_{1}$ mapping method with reference to TSE-IR for three concentrations of contrast agent. Mean and standard deviation values were calculated by placing an ROI on the central slice of the phantom. . . . . . . . . . . . . .

4.2 Table showing results of the cVFA $T_{1}$ mapping method with reference to TSE-IR for three concentrations of contrast agent. Mean and standard deviation values were calculated by placing an ROI on the central slice of the phantom. . . . . . . . . . . . . . .

4.3 Table showing results of MOLLI $T_{1}$ mapping with reference to TSEIR for three concentrations of contrast agent. Mean and standard deviation values were calculated by placing an ROI on the central slice of the phantom. . . . . . . . . . . . . . . .

4.4 Measured $[G d]$ results for the $T_{1 p r e}$-uVFA method. "Actual" columns represent reference values calculated using TSE-IR. . . . . . . . . . . 61

4.5 Slopes of the linear regression analysis of the mean, minimum, and

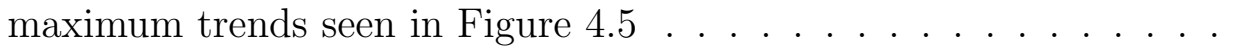


4.6 Measured $[G d]$ results for the $T_{1 p r e}$-cVFA method. "Actual" columns represent reference values calculated using TSE-IR. . . . . . . . . 63

4.7 Slopes of the linear regression analysis of the mean, minimum, and maximum trends seen in Figure 4.6. . . . . . . . . . . . . . .

4.8 Measured $[G d]$ results for the $T_{1 p r e}-$ LL method. "Actual" columns represent reference values calculated using TSE-IR. . . . . . . . . . .

4.9 Slopes of the linear regression analysis of the mean, minimum, and maximum trends seen in Figure 4.7 . . . . . . . . . . . . . 66

4.10 Measured $[G d]$ results for the BE-uVFA method. "Actual" columns represent reference values calculated using TSE-IR. . . . . . . . 68

4.11 Slopes of the linear regression analysis of the mean, minimum, and maximum trends seen in Figure 4.8. . . . . . . . . . . . . 68

4.12 Measured $[G d]$ results for the BE-cVFA method. "Actual" columns represent reference values calculated using TSE-IR. . . . . . . . 70

4.13 Slopes of the linear regression analysis of the mean, minimum, and maximum trends seen in Figure 4.9 . . . . . . . . . . . . . . 71

4.14 Measured $[G d]$ results for the BE-LL method. "Actual" columns represent reference values calculated using TSE-IR. . . . . . . . . . 72

4.15 Slopes of the linear regression analysis of the mean, minimum, and maximum trends seen in Figure 4.10 . . . . . . . . . . . . 73

4.16 Percent error plus and minus the standard deviation within ROI for slices 5-14 for the $T_{1 \text { pre }}$ method relative to slice $9 \ldots \ldots \ldots 74$

4.17 Percent error plus and minus the standard deviation within the ROI for slices 5-14 for the BE method relative to slice $9 \ldots \ldots \ldots$

5.1 Average percent error with respect to TSE-IR reference $T_{1}$ values. The above are calculated from Tables 4.1, 4.2, and 4.3. . . . . . . 79 
5.2 Average standard deviations for the three $T_{1}$ mapping methods. The above are calculated from Tables 4.1, 4.2, and 4.3. The values reported are the mean standard deviation values plus and minus one standard deviation . . . . . . . . . . . . . . . . . 80

5.3 Resulting average slopes for the results in Section 4.3. The values listed here reflect the overall accuracy of a given method. . . . . . . . 83

5.4 Resulting precision for the results in Section 4.3. The values listed here reflect the overall uniformity of a given method. . . . . . . . . 87

5.5 Resulting spread for the results in Section 4.3. The values listed here reflect the overall reproducibility of a given method. . . . . . . . .

5.6 The average percent error with respect to the central slice for all $[G d]$ quantification methods. The errors represent the standard deviation of reported percent errors across slices. . . . . . . . . . . . .

5.7 The average precision for all $[G d]$ quantification methods. The errors represents the standard deviation of reported percent errors calculated with respect to the central slice. . . . . . . . . . . . . . . . . . 


\section{List of Figures}

2.1 Diagram indicating the geometry of the precession of the angular momentum vector, $\boldsymbol{J}$ (and thus the magnetic dipole) about the static field $\boldsymbol{B}_{0} \ldots \ldots \ldots \ldots \ldots \ldots \ldots \ldots \ldots \ldots \ldots \ldots \ldots \ldots \ldots$

2.2 Energy splitting diagram indicating the parallel and antiparallel con-

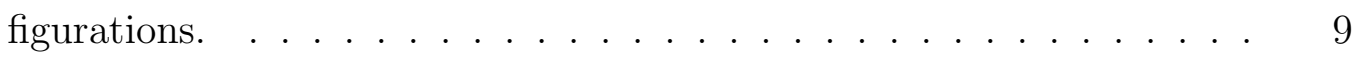

2.3 Simulated figure showcasing both the longitudinal and transverse relaxation of the magnetization. . . . . . . . . . . . . . . 14

2.4 Components integral to the evaluation of important pharmacokinetic parameters in DCE-MRI. An example given here is $K^{\text {trans }}$, the compartmental transfer coefficient which relates to vessel wall permeability. 19

2.5 Schematic of the two compartment exchange model. The total contrast agent concentration detected by the scanner is $[G d](t)$. The total $[G d]$ measured is composed of compartmental concentrations in the EES and plasma, denoted $[G d]_{e}(t)$ and $A I F(t)$, respectively. $\ldots \ldots 20$

2.6 Schematic of both the $T_{1 \text { pre }}$ and Bookend methods in a DCE-MRI

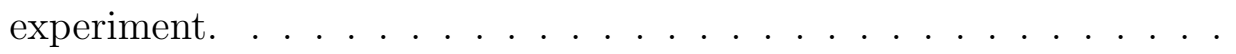


2.7 Experimentally determined $P\left(T_{1}\right)$ for several values of $T_{1}$ (dots). Squares represent the Bookend measurements of $P\left(T_{1}\right)$. The solid black line represents the linear approximation of $P$ used in the BE calibration. The dashed line represents $P$ as assumed by $T_{1 \text { pre }}$ calibra-

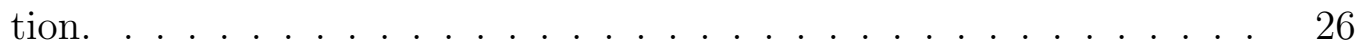

2.8 Comparison of measured FLASH signal to calibrated theoretical FLASH signal. Signal ratio is calculated as $S / P\left(T_{1}\right) F\left(\alpha, T R, T_{1}\right)$ from Eq. (2.27). A signal ratio closer to 1.00 represents a better calibration. 28

3.1 The phantom (a) and the phantom loaded into the head coil prior to translation into the magnet bore $(b) . \ldots \ldots \ldots . \ldots . \ldots$

3.2 Pulse sequence diagram for a 3D-FLASH acquisition. . . . . . . . 32

3.3 Axial images of the brain before the arrival of the bolus of contrast agent(A) and during the peak of signal enhancement due to presence of contrast agent (B). The signal enhancement curve of the area outlined by the circle $(\mathrm{C})$. Arrows indicate the position along the signal enhancement curve for both images. . . . . . . . . . . . . 34

3.4 Pulse sequence diagram for the traditional inversion recovery sequence. 36

3.5 Pulse sequence diagram indicating the generation of a stimulated echo. 40

3.6 Overview of the entire MOLLI pulse sequence. . . . . . . . . . . 44

3.7 Workflow of the $[G d]$ measurement experiment. The experiments begin

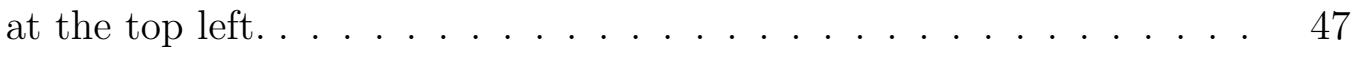

3.8 Diagram illustrating the different methods of $[G d]$ analysis. $\quad \ldots . .48$

4.1 All 18 slices of a typical uVFA $T_{1}$ map. Reference $T_{1}$ as measured by TSE-IR is denoted by the arrow on the colour bar. . . . . . . . . 52

4.2 All 18 slices of a typical cVFA $T_{1}$ map. Reference $T_{1}$ as measured by TSE-IR is denoted by arrow on colourbar. . . . . . . . . . . . 54 
4.3 All 18 slices of a typical MOLLI $T_{1}$ map. Reference $T_{1}$ as measured by TSE-IR is denoted by the arrow on the colour bar. . . . . . . . . . 56

4.4 A comparison of all three $T_{1}$ mapping techniques over a large range of $T_{1}$ values. Points represent mean $T_{1}$ and error bars represent plus and minus one standard deviation within the ROI. . . . . . . . . . . . . . 59

4.5 Trends of the $T_{1 p r e-\mathrm{uVFA}}$ analysis. . . . . . . . . . . . . . 62

4.6 Trends of the $T_{1 p r e-\mathrm{cVFA}}$ analysis. . . . . . . . . . . . 64

4.7 Trends of the $T_{1 p r e}$-LL analysis. . . . . . . . . . . . . . . 66

4.8 Trends of the BE-uVFA analysis. . . . . . . . . . . . . . . 69

4.9 Trends of the BE-cVFA analysis. . . . . . . . . . . . . 71

4.10 Trends of the BE-LL analysis. . . . . . . . . . . . . . . . 73

4.11 Interslice variation for the $T_{1 p r e}$-based methods for slices 5-14. Points represent mean percent error with respect to central slice. Error bars represent plus and minus one standard deviation. . . . . . . . . . 75

4.12 Interslice variation of the BE-based methods for slices 5-14. Points represent mean percent error with respect to central slice. Error bars represent plus and minus one standard deviation. . . . . . . . . . . 77

5.1 Average percent errors as a function of $T_{1}$ accuracy. . . . . . . . . . 79

5.2 Average standard deviation for the three $T_{1}$ mapping methods. . . . 81

5.3 Trends observed in the slope of $[G d]$ quantification as a function of $T_{1}$ accuracy. The horizontal dashed line represents the ideal slope of 1.00 corresponding to perfect agreement with reference values. The vertical dashed line separates the $T_{1 p r e}$ from the BE methods. . . . . . . . . 
5.4 Simulated signal curves as a function of $T_{1}$. The incorrectly calibrated curve represents a scaled down version of the true measured signal by a factor of 0.75 . This value is arbitrary and was chosen for demonstration only. The curves were both generated with parameters used in the clinical DCE protocol. $(\alpha=30$ degrees, $T R=6.5 \mathrm{~ms}) . \ldots . . . . \quad 85$

5.5 Trends in precision of the $T_{1 \text { pre }}$ and Bookend methods as a function of $T_{1}$ accuracy. The horizontal dashed line represents perfect agreement with the mean slope i.e. the $\mathrm{min} / \max$ slopes do not differ from the mean. The vertical dashed line separates $T_{1 p r e}$ from BE. . . . . . .

5.6 Images of the central $\left(9^{t h}\right)$ slice of the phantom for each $[G d]$ quantification method. The reference $[G d]$ is denoted by the arrow on the right. With increasing $T_{1}$ accuracy, images are noticeably more homogeneous. 90

5.7 Trends in the spread for both the $T_{1 \text { pre }}$ and BE methods as a function of $T_{1}$ accuracy. The vertical dashed line separates $T_{1 p r e}$ from BE. .

5.8 The average slopes along with 1-Precision (a) and Spread (b) as error bars. (a) represents the overall accuracy and image uniformity for a given method, while (b) represents the overall accuracy and reproducibility of results for a given method. . . . . . . . . . . . . . . . 96

5.9 Uniformity of $[G d]$ quantification for each method. Error bars represent standard deviation of reported percent errors with respect to the central slice. Horizontal dashed line represents perfect agreement with the central slice. . . . . . . . . . . . . . . . .

5.10 Average interslice image uniformity for each $[G d]$ quantification method. Error bars represent the standard deviation of reported percent errors with respect to the central slice. . . . . . . . . . . . . 100 


\section{List of Acronyms}

\begin{tabular}{ll} 
Acronyms & Definition \\
\hline \hline AIF & Arterial Input Function \\
BE & Bookend Method \\
DCE & Dynamic Contrast Enchanced Imaging \\
DSC & Dynamic Susceptibility Contrast Enhanced Imaging \\
EES & Extravascular Extracelluar Space \\
FISP & Fast Imaging with Steady State Precession \\
FLASH & Fast Low Angle Shot \\
FOV & Field Of View \\
IR & Inversion Recovery \\
LL & Look-Locker \\
MOLLI & Modified Look Locker Inversion Recovery \\
MRI & Magnetic Resonance Imaging \\
NMR & Nuclear Magnetic Resonance \\
& \\
& \\
& \\
& \\
&
\end{tabular}




\begin{tabular}{ll} 
RF & Radiofrequency \\
ROI & Region of Interest \\
SE & Spin Echo \\
STE & Steady State Free Precession \\
TE & Stimulated Echo \\
TI & Echo Time \\
TR & Inversion Time \\
TSE-IR & Repetition Time \\
VFA & Vurbo Spin Echo Inversion Recovery \\
cVFA & Corrected VFA \\
\hline \hline
\end{tabular}




\section{List of Symbols}

\section{Symbols Definition}

\begin{tabular}{ll}
\hline \hline$\alpha$ & flip angle \\
$\gamma$ & gyromagnetic ratio \\
$\boldsymbol{\mu}$ & magnetic dipole \\
$\tau_{p}$ & RF pulse duration \\
$\omega_{0}$ & Larmor frequency \\
$\Omega$ & frequency of rotating frame \\
$\boldsymbol{B}_{\mathbf{0}}$ & static, external magnetic field \\
$\boldsymbol{B}_{\mathbf{1}}$ & time varying magnetic field of the RF pulse \\
$\boldsymbol{J}$ & total angular momentum \\
$\boldsymbol{K}_{1}$ & compartmental transfer coefficient \\
$\boldsymbol{M}_{\text {G }}$ & net magnetization \\
&
\end{tabular}


$T_{1} \quad$ longitudinal or "spin-lattice" relaxation time

$T_{1 e f f} \quad$ effective longitudinal relaxation time

$T_{2} \quad$ transverse or "spin-spin" relaxation time

$T_{2}^{*} \quad$ apparent transverse relaxation time

$v_{e} \quad$ fractional extravascular extracellular volume

$v_{p} \quad$ fractional blood plasma volume 


\section{Chapter 1}

\section{Introduction}

The phenomenon of Nuclear Magnetic Resonance (NMR) was discovered by Isidor Rabi in 1938 [1]. For his work he was awarded the Nobel Prize in physics. Shortly thereafter, Felix Bloch and Edward Milles Purcell expanded on this work [2,3]. For many years, and even to this day, NMR has been an important technique in numerous areas of scientific research.

Originally, NMR was only applied to spectroscopic applications; imaging using NMR had not been invented yet. It was not until 1973, when Paul Lauterbur conceived the idea to spatially encode the NMR signal using magnetic field gradients [4], that imaging using NMR became possible. This technique made use of the change in Larmor frequency caused by the application of magnetic field gradients superimposed on to the static field to localize NMR signal. The first image, two spatially separated vials of water, gave birth to the technique known today as Magnetic Resonance Imaging (MRI).

Almost immediately, the benefits of MRI to the medical imaging field were noticed. MRI probes the proton spin-density of tissue to generate signal, providing superior soft tissue contrast than the imaging modalities of the day. This advantage holds true even today. Furthermore, MRI uses non-ionizing radiation to produce images.

MRI quickly became a diagnostic imaging staple in hospitals and many fields of 
MRI research have since exploded with activity. Traditional MRI has typically relied on conventional contrast mechanisms: $T_{1}, T_{2}$, and proton density (PD)-weighting. These types of images were excellent for providing anatomical information, but did not provide much in terms of functional or physiological information.

Hardware improvements and the development of signal enhancing contrast agents allowed new fields of MRI to emerge. Some of these fields yielded techniques that are able to probe underlying physiological processes in many parts of the body. A grouping of these techniques is known as Perfusion Weighted Imaging (PWI) and may be used to give a glimpse into the microvasculature of the tissue [5]. In the case of cerebral perfusion imaging, this helped in the identification and characterization of tumours. These techniques have grown and become more advanced allowing for better observation and quantification of physiologic processes in the brain and others parts of the body.

To understand MR-based perfusion imaging, it is important to understand the word "perfusion" itself. Perfusion has historically referred to the delivery of nutrients and elimination of waste in biological tissues. These actions are performed by the process of blood delivery to the capillary blood pool. More recently, with the advent of functional medical imaging, perfusion may be thought of as the volumetric flow rate of blood that supplies a subsection of tissue (e.g. a voxel). The tissue is a non-uniform object composed of many different constituent elements such as blood vessels, cells, etc. Due to this complex nature of tissue structure, perfusion is thought of as a scalar quantity; no attention is paid to the particular path taken by the blood through the maze of microvasculature capillaries, only the absolute value. Perfusion may be thought of as the "irrigation of tissue" [6]. Perfusion imaging is a very broad term, however. The purpose of many perfusion based techniques is not to measure the overall perfusion, but to measure perfusion-related parameters such as plasma volume or vessel wall permeability [5]. These parameters will affect the overall 
perfusion and vice-versa. The investigation of these parameters is lumped together under the umbrella term of perfusion imaging.

Generally speaking, most perfusion related MR techniques available today may be divided into two categories: techniques that require the introduction of an external contrast agent, and those that do not. The former will be the focus of this work and is currently the most clinically used form of MR-based perfusion imaging. In this category, there are two main imaging techniques. Both techniques are based on observing the temporal change in relaxation time after a contrast agent injection. Dynamic-Susceptibility Contrast Enhanced (DSC) MRI focuses on the changes in $T_{2}^{*}$ while Dynamic Contrast Enhanced (DCE) MRI focuses on the change in $T_{1}$ [5]. Both DSC-MRI and DCE-MRI will be explained in greater detail in Chapter 2.

For a long time, DCE-MRI had taken a back seat to DSC-MRI. This was due to hardware limitations, difficulty in modelling, and many other problems. DSC-MRI information has historically been easier to acquire and process, although its quantitative accuracy is somewhat controversial $[7,8]$. Recently, DCE-MRI has seen increased popularity. It is now widely used throughout the clinic and the area is a hotbed of research. DCE-MRI provides a unique look into the underlying physiological process in tumours and other tissues.

The focus of this work is related to DCE-MRI. As will be explained in detail later, quantification of contrast agent concentration is paramount in DCE-MRI. This work focuses on improving contrast agent quantification in phantoms. The results observed here are directly applicable to clinical DCE-MRI procedures. In order to quantify contrast agent concentration, accurate measurements of $T_{1}$ are required to calibrate the measured MR signal. This is done by calculating the value of a calibration factor that relates the measured signal strength to the theoretically predicted signal strength. In general, there are two commonly accepted ways to accomplish 
this. The first method, known as the $T_{1 \text { pre }}$ method, uses a single measurement of precontrast $T_{1}$, denoted $T_{1 \text { pre }}$, to calculate this calibration factor. In this method, the calibration factor is assumed to be constant in $T_{1}$. The second method, known as the Bookend method, defines the calibration factor more rigorously through the use of an additional measurement of $T_{1}$ after signal acquisition known as $T_{1 \text { post }}$. In the Bookend method, the calibration factor is allowed to vary with $T_{1}$, improving the correspondence between measured and theoretical signal strengths. This helps to increase the overall quantitative accuracy of the evaluation of contrast agent concentration.

This work is dedicated to the validation of the Bookend method. This work will show that the Bookend method provides better quantitative accuracy than the $T_{1 \text { pre }}$ method. In addition, the behaviour of both methods as increasingly accurate input $T_{1}$ measurements are used, will be investigated and quantified. 


\section{Chapter 2}

\section{Theory and Background}

This chapter provides the necessary background for understanding the rest of this work. It begins with an overview of the necessary MR physics needed to follow the subsequent chapters. Next, the concept of Dynamic Contrast Enhanced MRI is presented. The pathophysiological process of signal enhancement and the motivation behind the technique is explained and the importance of contrast agent quantification with time in this context is discussed. Finally, the two competing methods of quantification tested in this work are outlined and explained.

\subsection{Basic MR Theory}

\subsubsection{Classical Description of Nuclear Magnetic Resonance}

The phenomenon of nuclear magnetic resonance (NMR) may be explained in the classical limit with certain approximations. Nuclei with an odd number of protons and neutrons have a magnetic moment $\boldsymbol{\mu}$ due to a non-zero total angular momentum vector $\boldsymbol{J}$. In the classical interpretation, the nucleons are considered as "spinning particles" that also orbit the centre of the nucleus. This gives rise to orbital and spin angular momentum vectors, $\boldsymbol{L}$ and $\boldsymbol{S}$, respectively, which sum to form $\boldsymbol{J}[9]$. A 
"spinning charge" will also have a magnetic dipole moment defined as $\boldsymbol{\mu}=\gamma \boldsymbol{J}[10]$ where $\gamma$ is known as the "gyromagnetic ratio" and varies depending on the nucleus under consideration. For ${ }^{1} H$, the nucleus typically used to generate the MRI signal, $\gamma=2.675 \times 10^{8} \mathrm{~T}^{-1} \mathrm{~s}^{-1}$ or $\nsucc=\gamma / 2 \pi=42.574 \mathrm{MHz} / \mathrm{T}$ [10]. From henceforth, any reference to spins, nuclei, magnetic (dipole) moments, or protons within the context of MRI will be referring to the ${ }^{1} H$ nucleus. No other nucleus was used for imaging in this work.

When in the presence of a magnetic field $\boldsymbol{B}_{\mathbf{0}}$, the spins experience a torque which causes them to precess about the field at an angle $\theta$ with respect to $\boldsymbol{B}_{\mathbf{0}}[11]$. This is known as "free precession." In classical theory, torque is equal to the time derivative of the angular momentum and so [12]

$$
\frac{d \boldsymbol{J}}{d t}=\boldsymbol{\tau}=\boldsymbol{\mu} \times \boldsymbol{B}_{0}
$$

The frequency of this precession is denoted $\omega_{0}=d \phi / d t$ where $d \phi$ is the angle $d \boldsymbol{J}$ has rotated through in time $d t$ transverse to the direction of the magnetic field (see Figure 2.1).

By using an identity of the vector cross product this time derivative may be written as

$$
\frac{d J}{d t}=\mu B_{0} \sin \theta=\gamma J B_{0} \sin \theta
$$

and by the chain rule of calculus

$$
\frac{d J}{d t}=\frac{d J}{d \phi} \frac{d \phi}{d t}
$$

From Figure 2.1 it can be seen that $d J / d \phi=J \sin \theta$ and thus

$$
\frac{d \phi}{d t}=\frac{\gamma J B_{0} \sin \theta}{d J / d \phi}=\frac{\gamma J B_{0} \sin \theta}{J \sin \theta}=\gamma B_{0} .
$$




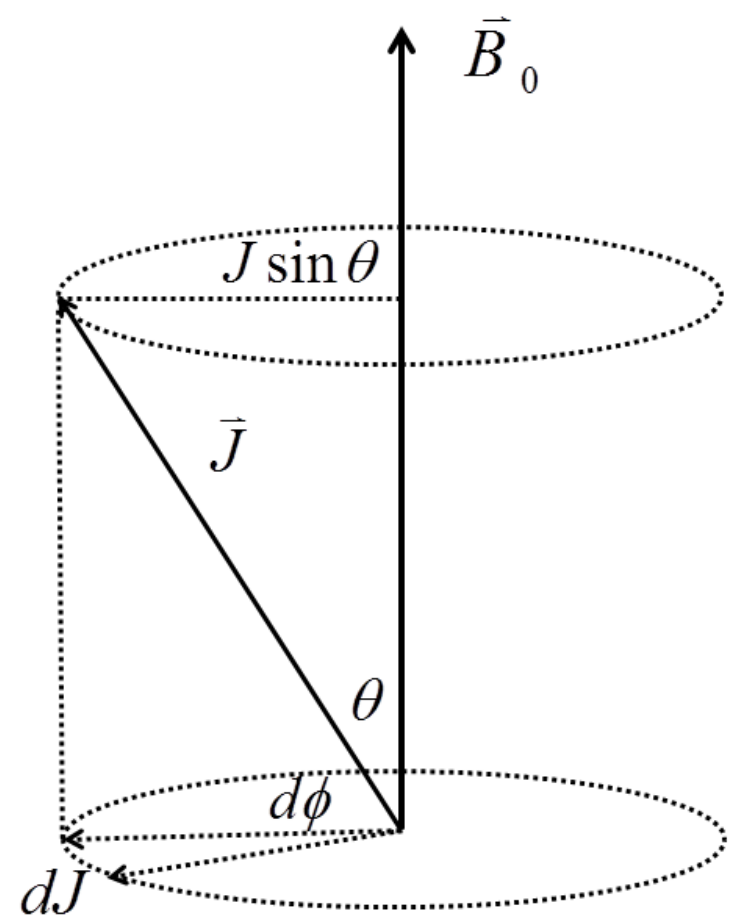

Figure 2.1: Diagram indicating the geometry of the precession of the angular momentum vector, $\boldsymbol{J}$ (and thus the magnetic dipole) about the static field, $\boldsymbol{B}_{\mathbf{0}}$.

The frequency of the precession of $\boldsymbol{\mu}$ about $\boldsymbol{B}_{\mathbf{0}}$ is then $\omega_{0}=\gamma B_{0}$ and is known as the "Larmor Frequency." The precession about $\boldsymbol{B}_{\mathbf{0}}$ is clockwise in direction such that $\boldsymbol{\omega}_{\mathbf{0}}=-\gamma \boldsymbol{B}_{\mathbf{0}}[12]$. This is a fundamental relationship within the fields of NMR and MRI.

\subsubsection{Origins of the MR Signal}

Without the presence of an external magnetic field, the spins in the system will be randomly oriented in space and the vector sum of all these magnetic moments, also 
known as the magnetization, will be zero

$$
\boldsymbol{M}=\sum_{i} \boldsymbol{\mu}_{i}=0, \quad\left(\boldsymbol{B}_{0}=0\right)
$$

However, in the presence of an external magnetic field, it has already been shown that the spins precess about $\boldsymbol{B}_{\mathbf{0}}$. A component of the spins will either align parallel or antiparallel with $\boldsymbol{B}_{\mathbf{0}}$, with the lower energy state being the preferred state. In this case, a net magnetization is possible and

$$
\boldsymbol{M}=\sum_{i} \boldsymbol{\mu}_{\boldsymbol{i}} \neq 0, \quad\left(\boldsymbol{B}_{\mathbf{0}} \neq 0\right)
$$

But just how many spins align with or against the external field? And consequently, how much magnetization is actually achieved? Descriptions of individual nuclei are not possible in the classical limit and fall within the domain of Quantum Mechanics. However, descriptions of the bulk properties of these spins in a macroscopic sense are employed. With this said, one particular rule will be borrowed from Quantum Mechanics to aid in the description.

Consider the famous Stern-Gerlach experiment performed by Otto Stern and Walther Gerlach in 1922 [13]. They showed that in the presence of a magnetic field, the orientations of the magnetic dipoles will not follow a continuous spectrum, but rather organize themselves into two distinct, discrete groups based on energy level. The lower energy population projects a component parallel to $\boldsymbol{B}_{\mathbf{0}}$ and is referred to as $N_{\uparrow}$. Conversely, the higher energy population projects a component antiparallel to $\boldsymbol{B}_{\mathbf{0}}$ and is referred to as $N_{\downarrow}$. Both parallel and antiparallel groups form the same angle with respect to the magnetic field direction.

The energies themselves are given as $E_{\uparrow}=-\frac{1}{2} \gamma \hbar B_{0}$ and $E_{\downarrow}=\frac{1}{2} \gamma \hbar B_{0}$ [11] for the parallel and antiparallel energy levels, respectively. According to Boltzmann 


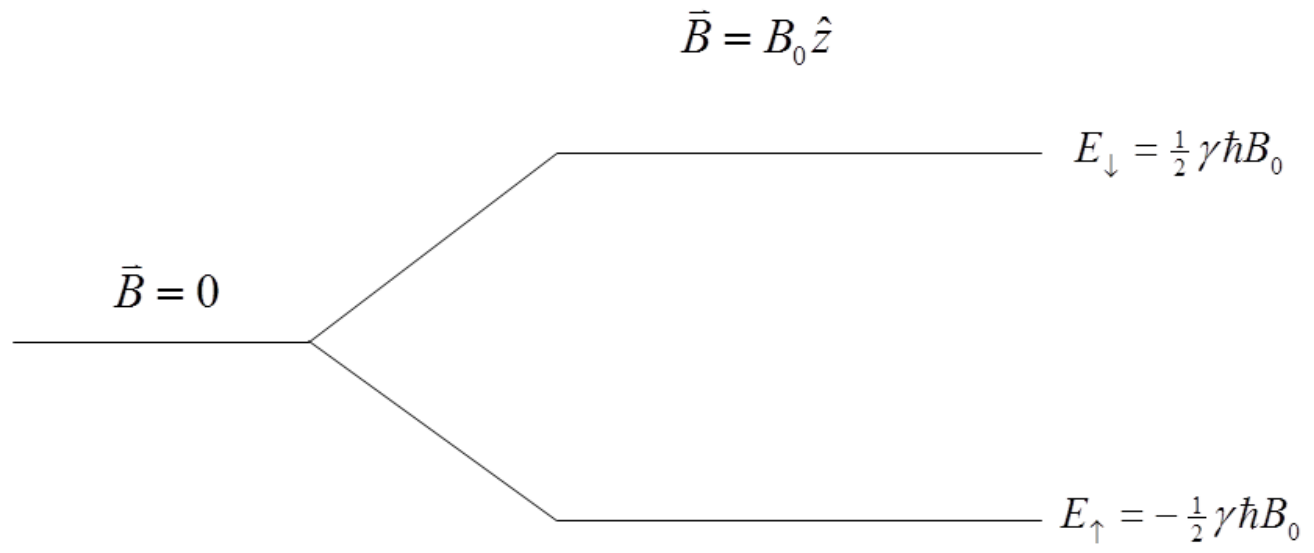

$\vec{B}=B_{0} \hat{z}$

$E_{\uparrow}=-\frac{1}{2} \gamma \hbar B_{0}$

Figure 2.2: Energy splitting diagram indicating the parallel and antiparallel configurations.

statistics, the fraction of a total number of particles in a particular energy state is given by $[14]$

$$
\frac{N_{i}}{N}=\frac{e^{-E_{i} / k_{B} T}}{\sum_{i} e^{-E_{i} / k_{B} T}},
$$

where $E_{i}$ is the energy of a given state, $N_{i}$ is the population of the $E_{i}$ energy state, $N$ is the total population of spins, $k_{B}$ is Boltzmann's constant, and $T$ is the absolute temperature. The ratio of the populations in the up versus down state is

$$
\frac{N_{\uparrow}}{N_{\downarrow}}=e^{\Delta E / k_{B} T}=e^{\gamma \hbar B_{0} / k_{B} T} .
$$

The argument of the exponential function is very small, and thus the ratio of the populations is close to unity. However, this small difference is key to imaging in MRI. A first order expansion performed on Eq. (2.8) results in

$$
\frac{N_{\uparrow}}{N_{\downarrow}} \approx 1+\frac{\gamma \hbar B_{0}}{k_{B} T} .
$$


Consequently, after rearrangement and approximation, the result is

$$
N_{\uparrow}-N_{\downarrow} \approx N \frac{\gamma \hbar B_{0}}{2 k_{B} T}
$$

The above equation is important and shows that there are, in fact, slightly more spins in the up state then in the down state. This is because the up state is at a lower energy than the down state, so statistically, more spins will occupy that state. Notice that by increasing the magnetic field strength, this difference increases (in turn creating a larger signal strength). For instance, at $B_{0}=1 \mathrm{~T},\left(N_{\uparrow}-N_{\downarrow}\right) / N \approx 3 \times 10^{-6}$. However, since there is on the order of Avogadro's number of spins in the system, a net magnetization large enough to manipulate to generate signal is obtained.

\subsubsection{Behaviour of $M$, the Rotating Frame, and $B_{1}$}

A classical explanation of NMR lends itself well to the description of the macroscopic net magnetization experienced in the presence of a large static magnetic field. The bulk magnetization $\boldsymbol{M}$ is the vector sum of all individual magnetic moments. At equilibrium, $\boldsymbol{M}$ will point along the static field direction which, by typical MRI orientation convention, is denoted the $\widehat{z}$ direction. This is because the transverse projections of the individual $\boldsymbol{\mu}$ cancel, since they are randomly oriented, while the components along $\widehat{z}$ do not cancel since more spins are parallel to $\boldsymbol{B}_{\mathbf{0}}$ than antiparallel. Explicitly, the equilibrium situation is $\boldsymbol{M}=M_{z} \widehat{z}=M_{0} \widehat{z}$.

In the laboratory frame, the equation of motion used to describe the motion of $\boldsymbol{M}$ in the presence of $\boldsymbol{B}_{\mathbf{0}}$ is [11]

$$
\frac{d \boldsymbol{M}}{d t}=\gamma \boldsymbol{M} \times \boldsymbol{B}_{\mathbf{0}}
$$

This is similar to the description of motion for $\boldsymbol{\mu}$ previously shown in Eq. (2.1). It 
is clear from Eq. (2.11) that, when $\boldsymbol{M}$ is at equilibrium (parallel to $\boldsymbol{B}_{\mathbf{0}}$ ), there is no precession about the static field. A static magnetization will not produce current in surrounding coils through the process of induction and therefore no signal is present. What is required is to move a component of $\boldsymbol{M}$ into the transverse plane $(\widehat{x}, \widehat{y})$ such that precession will occur.

Consider a rotating frame of reference with angular frequency $\Omega=\Omega \widehat{z}$ and orthogonal unit vectors $\left(\widehat{x^{\prime}}, \widehat{y^{\prime}}, \widehat{z^{\prime}}\right)$ related to the laboratory frame unit vectors $(\widehat{x}, \widehat{y}, \widehat{z})$. In this frame the equation of motion becomes [12]

$$
\left(\frac{d \boldsymbol{M}}{d t}\right)^{\prime}=\gamma \boldsymbol{M} \times\left(\boldsymbol{B}_{\mathbf{0}}+\frac{\boldsymbol{\Omega}}{\gamma}\right)
$$

where the prime of the left hand side of the equation indicates that it is in the rotating frame. Eq. (2.12) is nearly identical to Eq. (2.11) in the laboratory frame except that the static field has been replaced by the effective field $\boldsymbol{B}_{\boldsymbol{e f f}} \equiv \boldsymbol{B}_{\mathbf{0}}+\boldsymbol{\Omega} / \gamma$. The term $\Omega / \gamma$ is a "fictitious" field which arises as a result of the transformation between the rotating and stationary reference frames. If the frame of reference rotates with a frequency equal to the Larmor frequency (i.e. on resonance) such that $\Omega=-\gamma \boldsymbol{B}_{0}$ it is easy to see that the effect of the static field on $\boldsymbol{M}$ is canceled and $\boldsymbol{M}$ is stationary within the rotating frame.

Now consider the application of a circularly polarized, radiofrequency (RF) magnetic field perpendicular to the direction of the static field, termed $\boldsymbol{B}_{\mathbf{1}}$. The effective field experienced by $\boldsymbol{M}$ in the rotating frame will now be $\boldsymbol{B}_{\text {eff }}=\boldsymbol{B}_{\mathbf{0}}+\boldsymbol{\Omega} / \gamma+\boldsymbol{B}_{\mathbf{1}}$. Again, assuming the reference frame is on resonance, the effective field in the rotating frame reduces to the effect of the $\boldsymbol{B}_{\mathbf{1}}$ pulse only. The $\boldsymbol{B}_{\mathbf{1}}$ field will cause the "forced precession" of $\boldsymbol{M}$ about $\boldsymbol{B}_{\mathbf{1}}$ with frequency $\boldsymbol{\omega}_{\mathbf{1}}=-\gamma \boldsymbol{B}_{\mathbf{1}}$.

As a consequence of the forced precession of $\boldsymbol{M}$ due to the application of $\boldsymbol{B}_{\mathbf{1}}$, the magnetization vector will rotate through an angle $\alpha$ which is determined by the pulse 
envelope of $\boldsymbol{B}_{\mathbf{1}}$ as well as the pulse duration, $\tau_{p}$. This is known as the "flip angle" and is given by $[10-12]$

$$
\alpha=\gamma \int_{0}^{\tau_{p}} B_{1}(t) d t
$$

For instance, in the special case of a rect-type pulse envelope, the flip angle is given by $\alpha=\gamma B_{1} \tau_{p}$.

\subsubsection{Bloch Equations and Relaxation}

The well known Bloch Equations [11,12] are a set of equations that describe the time dependence of $\boldsymbol{M}$ due to the presence of $\boldsymbol{B}_{\mathbf{0}}$ after the application of an RF pulse. As shown in the previous section, the RF or $\boldsymbol{B}_{\mathbf{1}}$ field causes forced precession of $\boldsymbol{M}$ away from equilibrium. After the cessation of the RF pulse, a component of $\boldsymbol{M}$ will lie in the transverse plane and precess about $\boldsymbol{B}_{\mathbf{0}}$. This precession induces current in coils placed around the patient's body giving rise to the MR signal. However, once moved away from equilibrium, the magnetization will not remain there indefinitely. It will naturally relax back to equilibrium through several different relaxation mechanisms which will be described now.

The Bloch equations in all three directions are as follows [11,12]:

$$
\begin{gathered}
\frac{d M_{z}}{d t}=\gamma\left(\boldsymbol{M}(t) \times \boldsymbol{B}_{\mathbf{0}}\right)_{z}-\frac{M_{z}(t)-M_{0}}{T_{1}}, \\
\frac{d M_{x}}{d t}=\gamma\left(\boldsymbol{M}(t) \times \boldsymbol{B}_{\mathbf{0}}\right)_{x}-\frac{M_{x}(t)}{T_{2}}, \\
\frac{d M_{y}}{d t}=\gamma\left(\boldsymbol{M}(t) \times \boldsymbol{B}_{\mathbf{0}}\right)_{y}-\frac{M_{y}(t)}{T_{2}} .
\end{gathered}
$$

The last term on each of the three components is the relaxation term. These terms describe how the longitudinal component of the magnetization, $M_{z}$ regrows back 
to equilibrium and how the transverse components, $M_{x}$ and $M_{y}$, are eventually destroyed. Using the (on resonance) rotating frame, to make visualization simpler, these equations reduce to

$$
\begin{gathered}
\frac{d M_{z^{\prime}}}{d t}=-\frac{M_{z^{\prime}}(t)-M_{0}}{T_{1}}, \\
\frac{d M_{x^{\prime} y^{\prime}}}{d t}=-\frac{M_{x^{\prime} y^{\prime}}(t)}{T_{2}},
\end{gathered}
$$

where $M_{x^{\prime} y^{\prime}}$ is a complex function defined as $M_{x^{\prime} y^{\prime}}=M_{x^{\prime}}+i M_{y^{\prime}}$ [12]. The behaviour of the magnetization with time can be understood by solving the above differential equations which give:

$$
\begin{gathered}
M_{z^{\prime}}(t)=M_{0}\left(1-e^{-t / T_{1}}\right)+M_{z^{\prime}}(0) e^{-t / T_{1}}, \\
M_{x^{\prime} y^{\prime}}(t)=M_{x^{\prime} y^{\prime}}(0) e^{-t / T_{2}}
\end{gathered}
$$

Here, $M_{z^{\prime}}(0)$ and $M_{x^{\prime} y^{\prime}}(0)$ represent the initial magnetization in the longitudinal and transverse directions, respectively, immediately after RF excitation. It is clear from Eq. (2.19) and Eq. (2.20) that the relaxation in the longitudinal direction is characterized by an exponential regrowth with time constant $T_{1}$ and the relaxation in the transverse direction is characterized by exponential decay with time constant $T_{2}$, as shown in Figure 2.3.

$T_{1}$ relaxation occurs for the same reason individual spins reorient themselves in the presence of an external field. After the application of an RF pulse, a component of the magnetization will be in the transverse plane away from equilibrium. Thus, it will naturally relax back to equilibrium since this represents a lower energy state. The rate at which this occurs is determined by $T_{1}$.

$T_{2}$ relaxation occurs due to dephasing of spins in the transverse plane after excitation [12]. Local field inhomogeneities due to, for example, the presence of other nuclear magnetic moments, cause some spins to experience different field strengths. 


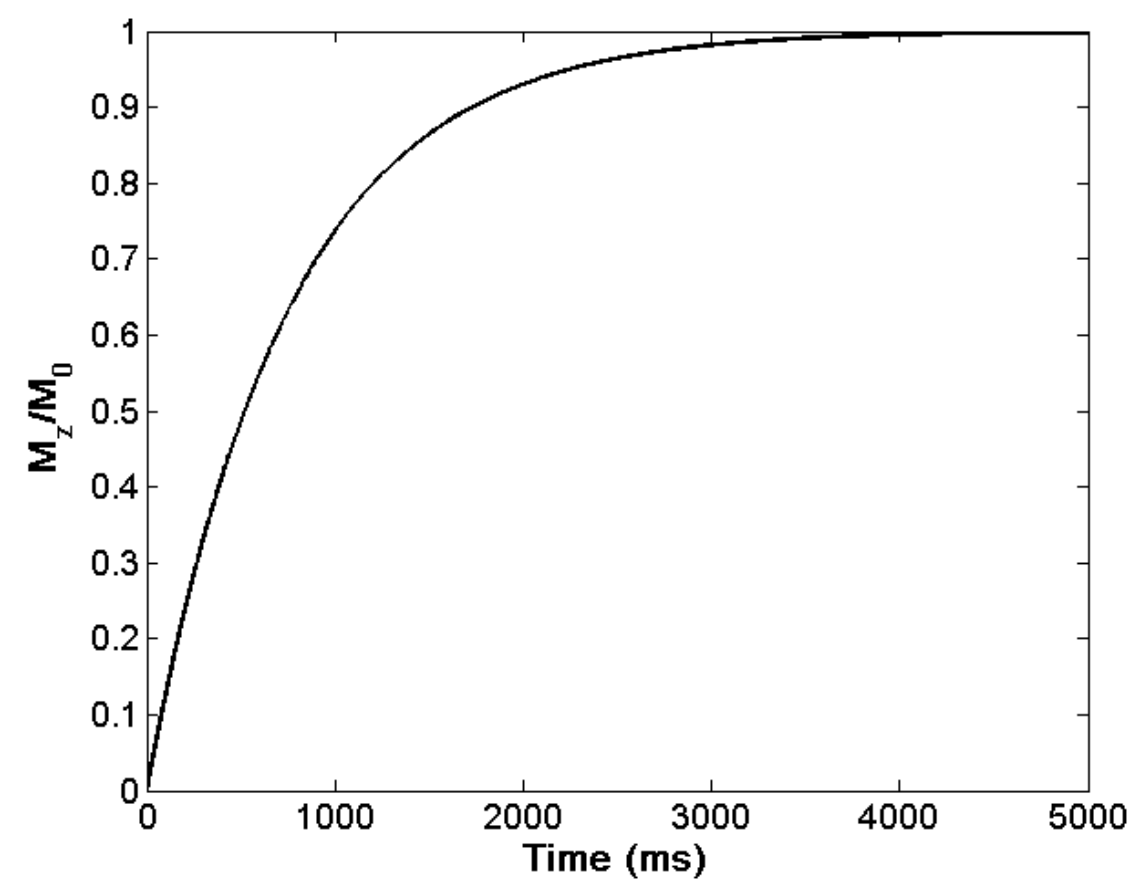

(a) Simulated longitudinal relaxation after a 90 degree RF pulse. $T_{1}=750 \mathrm{~ms}$.

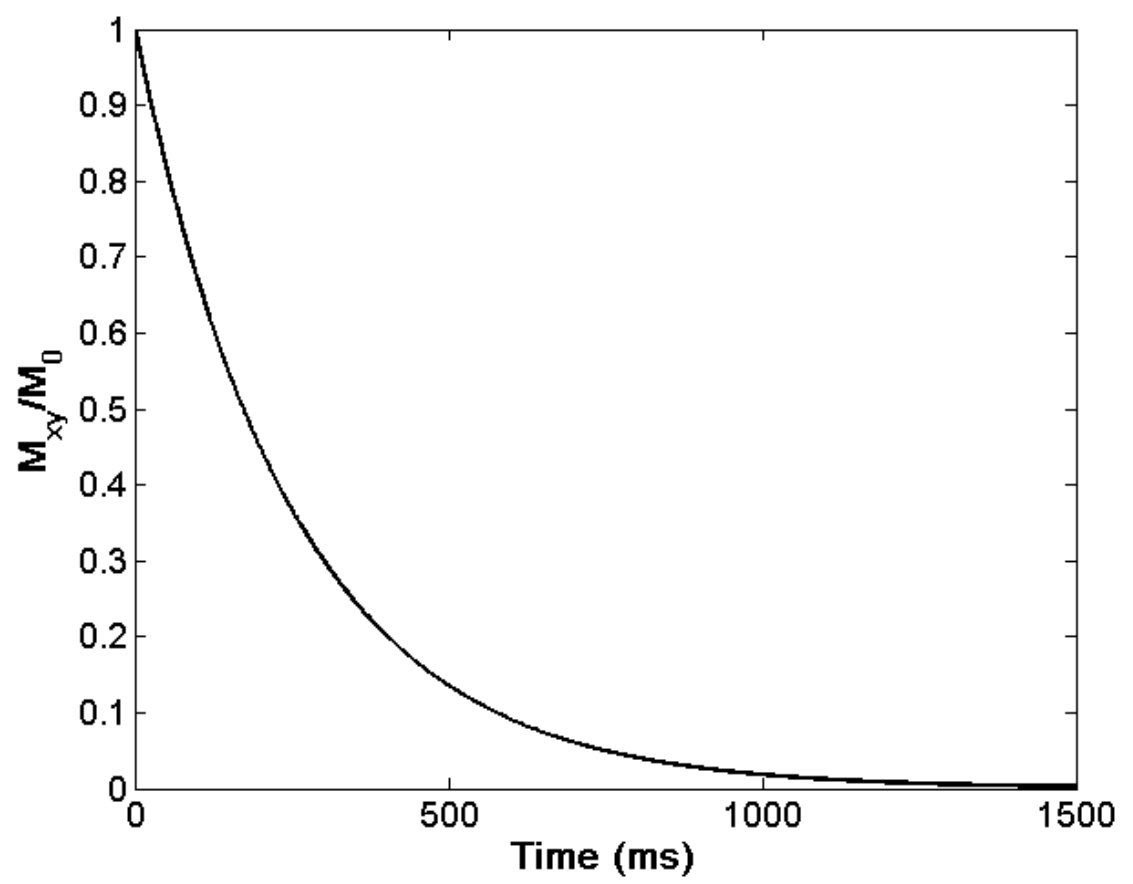

(b) Simulated transverse relaxation after a 90 degree pulse. $T_{2}=250 \mathrm{~ms}$.

Figure 2.3: Simulated figure showcasing both the longitudinal and transverse relaxation of the magnetization. 
This contributes to random, temporary differences in precession frequencies, causing the spins to become out of phase. Over time, these transient effects will cause the many spins in the sample to eventually become irreversibly out of phase, leading to a destruction of signal. In addition to this intrinsic and irreversible dephasing of the spins, there is a further systematic dephasing of the magnetization by small static field inhomogeneities, normally dominated by imperfections in the applied external magnetic field. These losses are reversible. The relaxation rate corresponding to these losses will be denoted $T_{2}^{\prime}$. The overall relaxation rate in the transverse plane, $T_{2}^{*}$, may be calculated as follows:

$$
\frac{1}{T_{2}^{*}}=\frac{1}{T_{2}}+\frac{1}{T_{2}^{\prime}} .
$$

It is possible to recover signal strength from losses due to static field inhomogeneities by reversing this dephasing through the use of an "echo" [12]. The mechanics of echo formation will not be discussed here.

\subsection{Dynamic Contrast Enhanced MRI}

\subsubsection{Introduction to DCE-MRI}

Dynamic Contrast Enhanced (DCE) MRI is an imaging technique, used to rapidly track in time, the distribution of a contrast agent as it moves throughout a tissue or organ of interest, without the use of ionizing radiation. Many different physiological factors are involved in the perfusion and uptake of the contrast agent [15]. By monitoring the evolution of the concentration of the contrast agent with time, conclusions about some of these overarching physiological processes, can be drawn $[5,15]$. This project is applicable to all areas of DCE-MRI research, but gliomas (a type of brain tumour) are of special interest. In gliomas, as well as most other cancerous 
lesions, the rapid, unregulated growth of cells, eventually leads to an oxygen deficiency caused by a lack of adequate microvasculature supporting the tumour. In time, angiogenesis occurs in order to sufficiently supply the uncontrolled growth of the tumour. However, the resulting vasculature is abnormal with respect to healthy vascular tissue. Tumours tend to have more vasculature than normal tissue overall. In addition, the vasculature structure is characterized by random, tortuous paths with a disproportionately high amount of branching and shunting $[15,16]$. The vasculature is functionally abnormal as well; the poorly formed vessels allow the leakage of contrast agent into the extracellular extravascular space (EES) much more freely than normal blood vessels $[5,15]$. Since DCE-MRI is able to reflect some of the overall physiological process in the microvasculature of tissues, it may be used to obtain valuable information about the microvasculature of tumours. Information about the tumour extracted using DCE-MRI, such as blood volume and vessel permeability, may be directly linked to tumour structure and function $[17,18]$. Thus, physiological parameter estimation conducted with DCE-MRI is of great potential use in the fields of radiology and oncology, among others. DCE-MRI may also be useful for non-invasive treatment monitoring of tumours [19,20] and tumour grading [21].

\subsubsection{Fundamentals of DCE-MRI}

Most MR contrast agents cause a decrease in $T_{1}$ and $T_{2}^{*}$ in the surrounding tissue. The decrease in $T_{1}$ happens in the immediate vicinity of the agent, while the decrease in $T_{2}^{*}$ happens over a much larger spatial range due to the susceptibility induced gradients which assist in the dephasing of spins [5]. There are two main contrast agent based methods for analyzing perfusion characteristics in tissue, of which DCE-MRI is one. The other is known as Dynamic Susceptibility Contrast-enhanced (DSC) MRI. DSC-MRI is based on the decrease in $T_{2}^{*}$ in the surrounding tissue as the contrast agent is introduced through the use of $T_{2}^{*}$-weighted MR imaging [5]. DSC-MRI lends 
itself well to the measurement of tissue perfusion parameters such as blood volume or flow. This is because in a highly constricted space such as a vessel, the contrast agent introduces an inhomogeneous distribution of field perturbations, exaggerating the $T_{2}^{*}$ decay. On the other hand, DCE-MRI uses the change in longitudinal relaxation caused by the contrast agent through the use of $T_{1}$-weighted imaging [5]. The $T_{1}$ decreasing effects, explained later, happen at very small distances away from the contrast agent molecule. Thus, in situations where the contrast agent has the freedom to exchange between intravascular (plasma) and extravascular (EES) compartments, it will be more homogeneously distributed, allowing many spins to access it, exaggerating the overall $T_{1}$ decrease. As explained in more depth in Section 2.2.3, this allows DCE-MRI to probe aspects of the microvasculature such as vessel wall permeability and compartment fractional volume. Therefore, while very similar, DSC-MRI and DCE-MRI have distinct sets of advantages and complement one another.

The contrast agent used in this study uses a gadolinium-based molecule known chemically as gadobutrol $\left(\mathrm{C}_{18} \mathrm{H}_{31} \mathrm{GdN}_{4} \mathrm{O}_{9}\right)$ and marketed commercially by Bayer pharmaceuticals as "Gadovist" [22]. Gadovist is a non-protein binding contrast agent with a paramagnetic gadolinium ion at the centre of a biologically inert "cage." The molecule is free to pass through the microvasculature endothelium from the blood plasma into the extracellular space outside of the vessel. The molecule will not pass through cell membranes into intracellular space. The gadolinium ion has several unpaired electrons which cause local field inhomogeneities which are time dependent due to tumbling and rotation of the Gadovist molecule. If these inhomogeneity fluctuations occur at the resonant frequency, this causes the relaxation of nearby excited protons [23]. This contributes to a shortening of the longitudinal relaxation time constant, $T_{1}$. In a $T_{1}$-weighted $\mathrm{MR}$ image, the primary source of image contrast is determined by the differences in $T_{1}$ in the field of view. A shorter $T_{1}$ will create a larger signal, appearing brighter in the image. Using $T_{1}$-weighted imaging sequences 
with high temporal resolution, it is possible to track the flow of the contrast agent through the brain by tracking these changes in signal strength.

It has been shown in the literature that over a wide range of concentrations, the change in $1 / T_{1}$ with respect the longitudinal relaxation rate prior to injection of contrast agent, is directly proportional to the concentration of the contrast agent present $[24,25]$ :

$$
\frac{1}{T_{1}}-\frac{1}{T_{1 p r e}}=r_{1}[G d]
$$

where $T_{1 \text { pre }}$ is the baseline longitudinal relaxation time prior to injection, $[G d]$ is the contrast agent concentration, and $r_{1}$ is known as the "relaxivity." For Gadovist at 3 Tesla and body temperature, $r_{1}=3.9 \mathrm{mM}^{-1} \mathrm{~s}^{-1}$, approximately $[22,26]$. Therefore, by monitoring how the longitudinal relaxation time changes with respect to the baseline, the concentration of the contrast agent may be evaluated. By converting the raw signal data collected during the scan, it is possible to produce parametric, fourdimensional concentration maps for the brain. The actual process of this conversion is explained in detail in Section 2.3. Concentration maps are one of three components needed to produce the biologically relevant parameters talked about earlier. In DCEMRI, the other two components are: the arterial input function (AIF), which describes the time evolution of $[G d]$ in the blood plasma, as well as a pharmacokinetic model that describes the exchange of contrast agent molecules between blood plasma and EES.

\subsubsection{Vascular Input and Kinetic Modelling}

Kinetic parameter modelling in DCE-MRI is a highly controversial subject. There are many different ways to model the exchange of contrast agent in tissue so that one may examine the underlying physiological processes. Each different model has its own advantages and disadvantages. At The Ottawa Hospital, the General Kinetic Model 


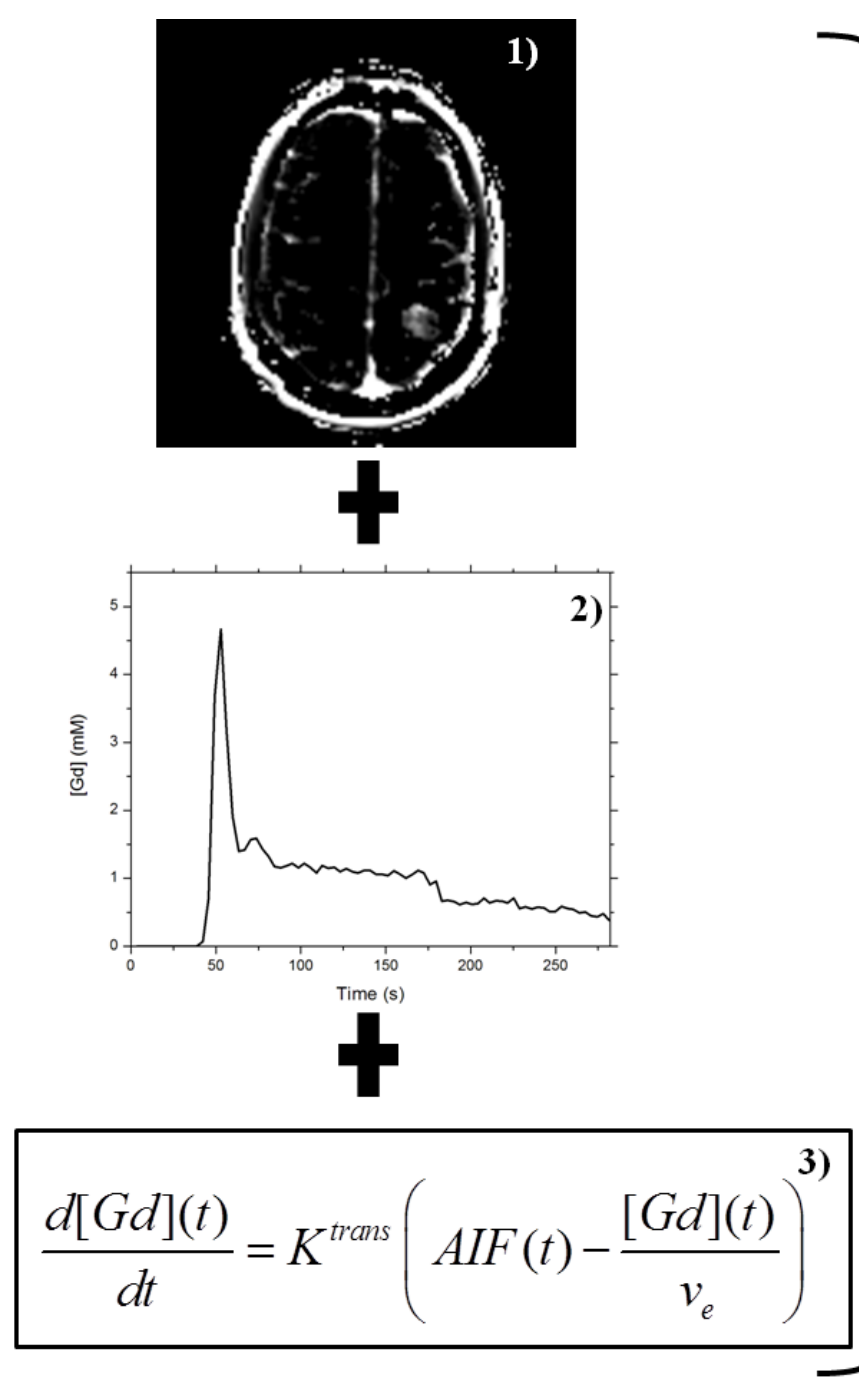

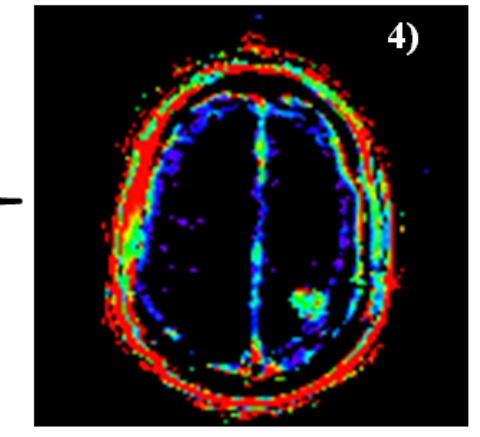

1) [Gd] vs. Time Map

2) Arterial Input Function (AIF)

3) Pharmacokinetic Model

4) Kinetic Parameter Maps (e.g. $\mathbf{K}^{\text {trans }}$ )

Figure 2.4: Components integral to the evaluation of important pharmacokinetic parameters in DCE-MRI. An example given here is $K^{\text {trans }}$, the compartmental transfer coefficient which relates to vessel wall permeability. 
(GKM) or Modified Tofts Model $[15,27]$ is used. This model is a two compartment model that takes into account the contribution of contrast agent concentration in both the blood plasma and EES as the source of the total $[G d]$ signal measured by the MR scanner. The entire voxel is made up of "tissue." This tissue may be subdivided into three categories: intracellular space (no contrast agent is found here), intravascular extracellular space (blood plasma), and extravascular extracellular space (EES, i.e. everywhere else).

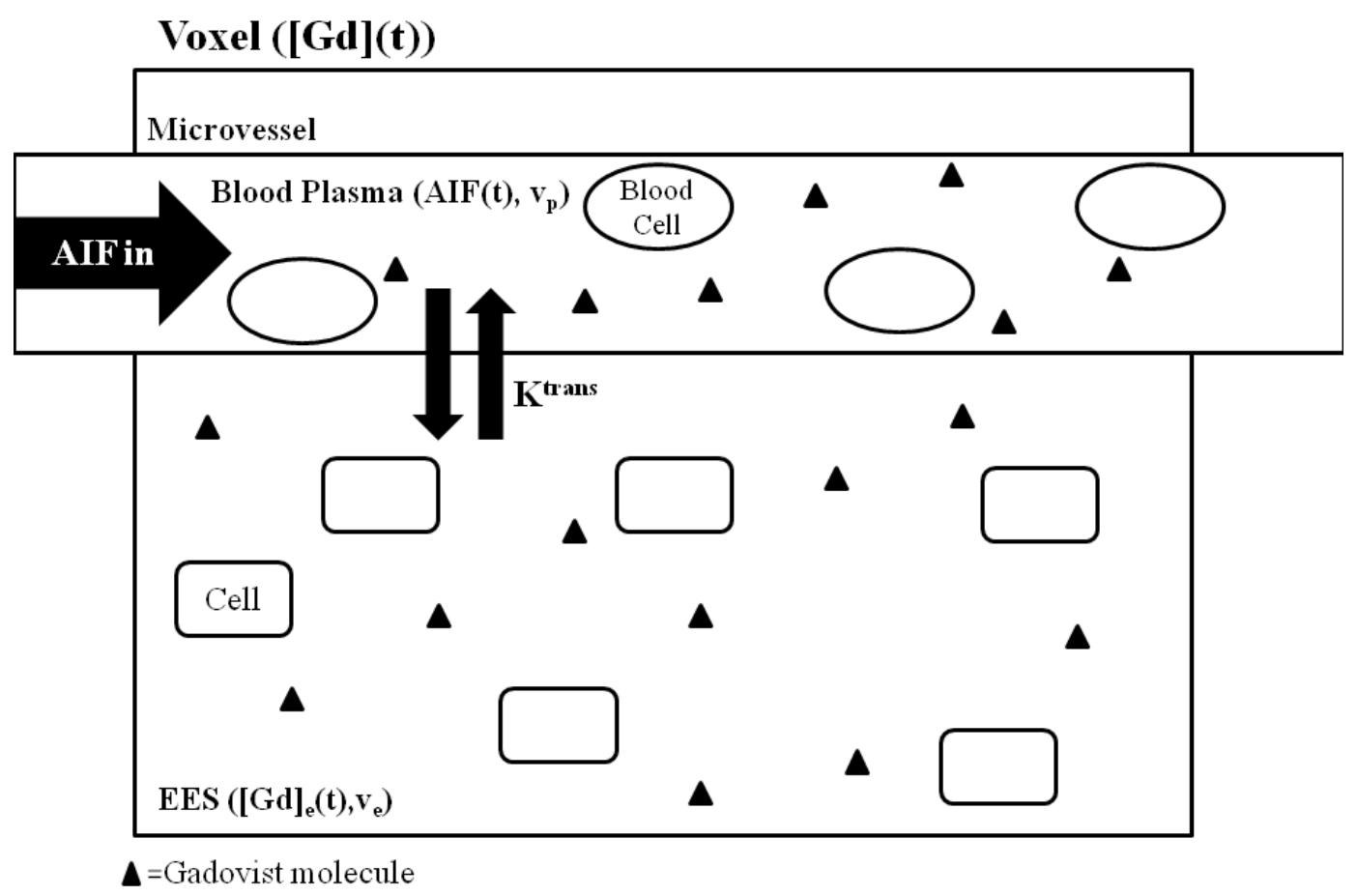

Figure 2.5: Schematic of the two compartment exchange model. The total contrast agent concentration detected by the scanner is $[G d](t)$. The total $[G d]$ measured is composed of compartmental concentrations in the EES and plasma, denoted $[G d]_{e}(t)$ and $A I F(t)$, respectively.

Let the parameters $[G d](t),[G d]_{e}(t)$, and $A I F(t)$ represent the contrast agent concentration in the entire voxel, the EES, and the blood plasma, respectively. The 
parameters $v_{p}$ and $v_{e}$ represent the volume fraction of the plasma and EES, respectively, relative to the total volume of the tissue voxel. The flux of contrast agent into the EES from the blood is dependent on the difference in concentration between the two compartments and the permeability of the vessel wall $\left(K^{\text {trans }}\right)$. The flux is given by $[27]$ :

$$
\frac{d[G d]_{e}(t)}{d t}=K^{\operatorname{trans}} \frac{\left(A I F(t)-[G d]_{e}(t)\right)}{v_{e}} .
$$

The total amount of contrast agent concentration in the tissue, which is measured, is related to its subcomponents by the following [15]

$$
[G d](t)=v_{e}[G d]_{e}(t)+v_{p} A I F(t)
$$

Eq. (2.23) is a linear, first order differential equation. Upon solving this equation, the following is obtained

$$
[G d]_{e}(t)=\frac{K^{\text {trans }}}{v_{e}} \int_{0}^{t} A I F\left(t^{\prime}\right) e^{-K^{\text {trans }}\left(t-t^{\prime}\right) / v_{e}} d t^{\prime}
$$

By substituting Eq. (2.25) into Eq. (2.24) this yields the model

$$
[G d](t)=v_{p} A I F(t)+K^{\text {trans }} \int_{0}^{t} A I F\left(t^{\prime}\right) e^{-K^{\operatorname{trans}\left(t-t^{\prime}\right) / v_{e}}} d t^{\prime}
$$

As previously mentioned, $[G d](t)$ is a measured quantity and, therefore, a known parameter. $A I F(t)$ is known as the vascular input function and is also a known parameter in DCE-MRI. However the method by which it is obtained is not as straight forward as the measurement of $[G d](t)$. There are many different methods in use to determine the vascular input. Some methods use information in the $[G d](t)$ maps to determine the vascular input by measuring the blood plasma concentration in a major vein or artery that supplies blood to the tissue or organ of interest [15]. Some methods 
assume knowledge of the vascular input by using empirically gathered data about the vascular input of a subset of the population. This is known as a "population AIF" $[28,29]$. Others use phase information gathered during the experiment to determine the vascular input $[30,31]$. Eq. (2.26) makes use of the measured values of $[G d](t)$ and $A I F(t)$ to fit for the parameters $K^{\text {trans }}, v_{e}$, and $v_{p}$.

\subsection{Concentration Quantification of Gadolinium- based Contrast Agent}

\subsubsection{DCE Signal Acquisition}

Most DCE acquisitions are performed using a pulse sequence from the spoiled gradient echo family of MRI sequences. These allow for very rapid, $T_{1}$-weighted images to be produced. As such, these sequences are good candidates for tracking contrast agent evolution in time, as is required in DCE-MRI. The particular pulse sequence used in this work is a Siemens Fast Low Angle Shot (FLASH) sequence [32,33]. The signal strength equation for this type of sequence may be written as [34]:

$$
S=P\left(T_{1}\right) F\left(\alpha, T R, T_{1}, T E, T_{2}^{*}\right)=P\left(T_{1}\right) \sin \alpha \frac{1-e^{-T R / T_{1}}}{1-e^{-T R / T_{1}} \cos \alpha} e^{-T E / T_{2}^{*}}
$$

where $T R$ and $T E$ are the repetition and echo times of the pulse sequence, respectively, and $\alpha$ is the flip angle. $P\left(T_{1}\right)$ is a scaling/correction factor that attempts to correct for divergence between the behaviour of the actual (measured) signal and the theoretical function, $F$, due to system imperfections such as RF transmit inhomogeneities [34]. Typically, as is the case in this work, $T E$ is made to be very short such that $T E \ll T_{2}^{*}$ to minimize $T_{2}^{*}$ losses and generate $T_{1}$-weighting. Consequently, $T_{2}^{*}$ effects will be ignored going forward. 
Once the raw DCE signal acquisition has been completed, the next step is to relate the signal enhancement due to the presence of the gadolinium $(\mathrm{Gd})$ based MRI contrast agent, to the concentration of the contrast agent, written $[G d]$. Theoretically, this is as simple as working backwards from the measured data, $S$, and extracting $T_{1}$ using Eq. (2.27). One may then use these $T_{1}$ values to convert to $[G d]$ through Eq. (2.22). However, one must fully calibrate the right hand side of Eq. (2.27) to obtain accurate results. To do this, the factor $P\left(T_{1}\right)$ must be fully accounted for.

\subsubsection{The Conventional $T_{1 p r e}$ Method}

The conventional way for converting between signal strength and $[G d]$ is to directly measure the baseline $T_{1}$ prior to injection and use this to calibrate the signal strength equation. Then one may work backwards from the measured signal strength to extract the $T_{1}$ changes in time. Since with this approach a single pre-injection $T_{1}$ map is used, the technique will be referred to as the $T_{1 \text { pre }}$ method throughout this work.

If $T_{1}$ and $S$ are both measured before the introduction of the Gd-based contrast agent, then $P\left(T_{1}\right)$ may be estimated by rearranging Eq. (2.27) to get

$$
P_{\text {pre }}=P\left(T_{1 p r e}\right)=\frac{S_{\text {pre }}}{F\left(\alpha, T R, T_{1 p r e}\right)} .
$$

$\alpha$ and $T R$ are both pulse sequence input parameters that are set by the operator and therefore known. Thus, $P\left(T_{1 \text { pre }}\right)$ may be calculated using input and measured parameters. However, with the $T_{1 \text { pre }}$ method, $P\left(T_{1}\right)$ is assumed not to vary with $T_{1}$ and is treated as constant. This is a cause for concern since it has been shown empirically [34] that $P\left(T_{1}\right)$ is not constant, but changes approximately linearly in $T_{1}$. By assuming the correction factor is constant, a certain amount of rigidity in $T_{1}$ estimation is introduced, and any divergence between the behaviour of the measured signal and theory remains unaccounted for. This is especially true at low values of 
$T_{1}$ (i.e. high $[G d]$ ).

\subsubsection{The Bookend Method}

In an attempt to correct for the shortcomings of the conventional $T_{1 \text { pre }}$ method, a technique was introduced by Cron et al. [34] that makes use of an additional post injection measurement of $T_{1}$ to better account for signal strength variation due to $T_{1}$ changes. In this procedure, which is called the Bookend $(\mathrm{BE})$ method, $P\left(T_{1}\right)$ is assumed to change linearly with $T_{1}$ during the DCE-MRI experiment. As in the $T_{1 \text { pre }}$

Bookend Method

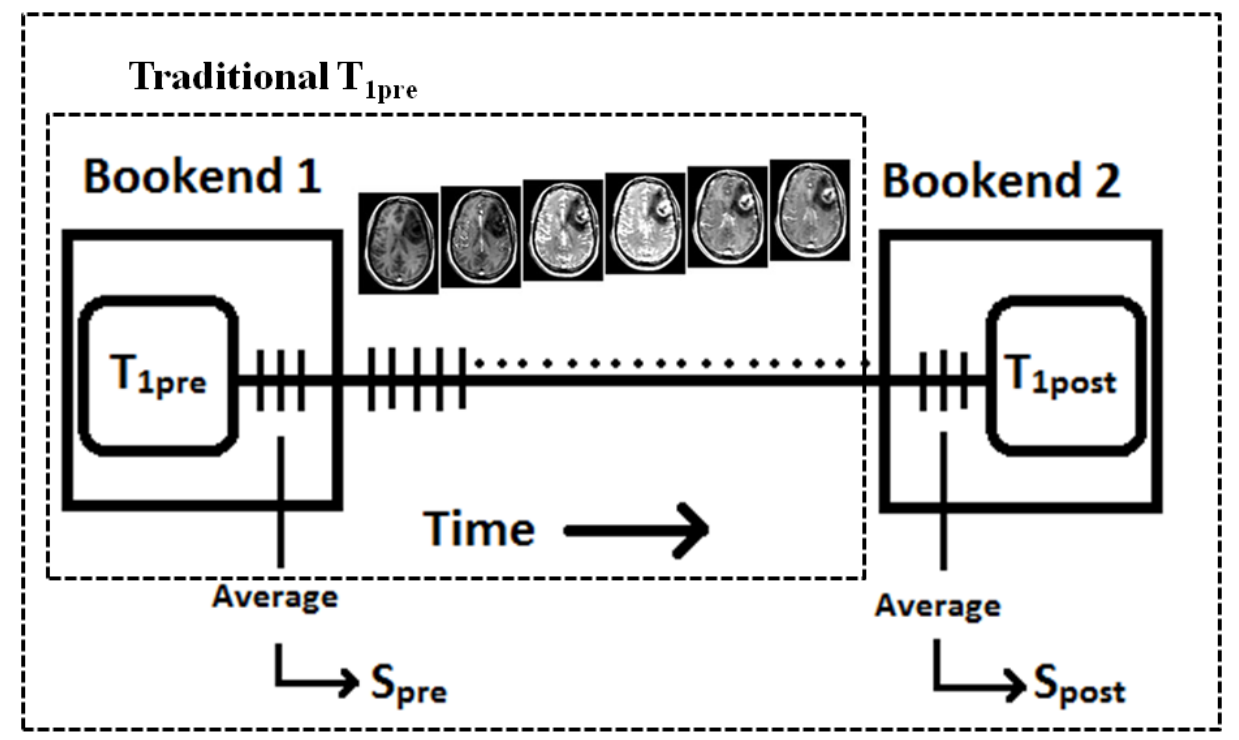

Figure 2.6: Schematic of both the $T_{1 \text { pre }}$ and Bookend methods in a DCE-MRI experiment.

method, $P\left(T_{1}\right)$ prior to injection may be determined from measured signal strengths and $T_{1}$ via Eq. (2.28). Similarly, $P\left(T_{1}\right)$ after $[G d]$ reaches a quasi-equilibrium state 
with time, or plateau, may be determined through the use of the second $T_{1}$ measurement

$$
P_{\text {post }}=P\left(T_{1 \text { post }}\right)=\frac{S_{\text {post }}}{F\left(\alpha, T R, T_{1 \text { post }}\right)}
$$

The two experimentally determined points $P_{\text {pre }}$ and $P_{\text {post }}$ are then used to evaluate $P\left(T_{1}\right)$. This is done on a voxel by voxel basis. $P\left(T_{1}\right)$ is assumed to be of the form

$$
P\left(T_{1}\right)=m T_{1}+P_{0}
$$

where

$$
m=\frac{P_{\text {pre }}-P_{\text {post }}}{T_{1 p r e}-T_{1 p o s t}},
$$

and

$$
P_{0}=\frac{P_{\text {post }} T_{1 \text { pre }}-P_{\text {pre }} T_{1 p o s t}}{T_{1 \text { pre }}-T_{1 \text { post }}} .
$$

With this estimate of $P\left(T_{1}\right)$, one may fully calibrate the signal strength equation and work backwards from the measured signal strength to extract $T_{1}$. Once the $T_{1}$ information has been extracted from the raw signal strength images, it is relatively straightforward to convert these images to $[G d]$ maps via Eq. (2.22).

\subsubsection{Justification for the Use of the Bookend Method}

As mentioned previously, the purpose of the Bookend Method is to more accurately relate the observed signal strength for a FLASH sequence to the concentration of contrast agent present in the tissue by introducing a calibration factor, $P\left(T_{1}\right)$, which is considered to vary linearly with $T_{1}$. Recall that with the $T_{1 p r e}$ method, $P\left(T_{1}\right)$ is calculated for a single value of $T_{1}$ and assumed to be constant for all $T_{1}$. With the Bookend method $P\left(T_{1}\right)$ is determined at two time points with different $T_{1}$ values and assumed to follow a straight line with respect to $T_{1}$ (but not necessarily with time). As can be seen from Figure 2.7, $P\left(T_{1}\right)$ is an approximately linear function of $T_{1}$. 


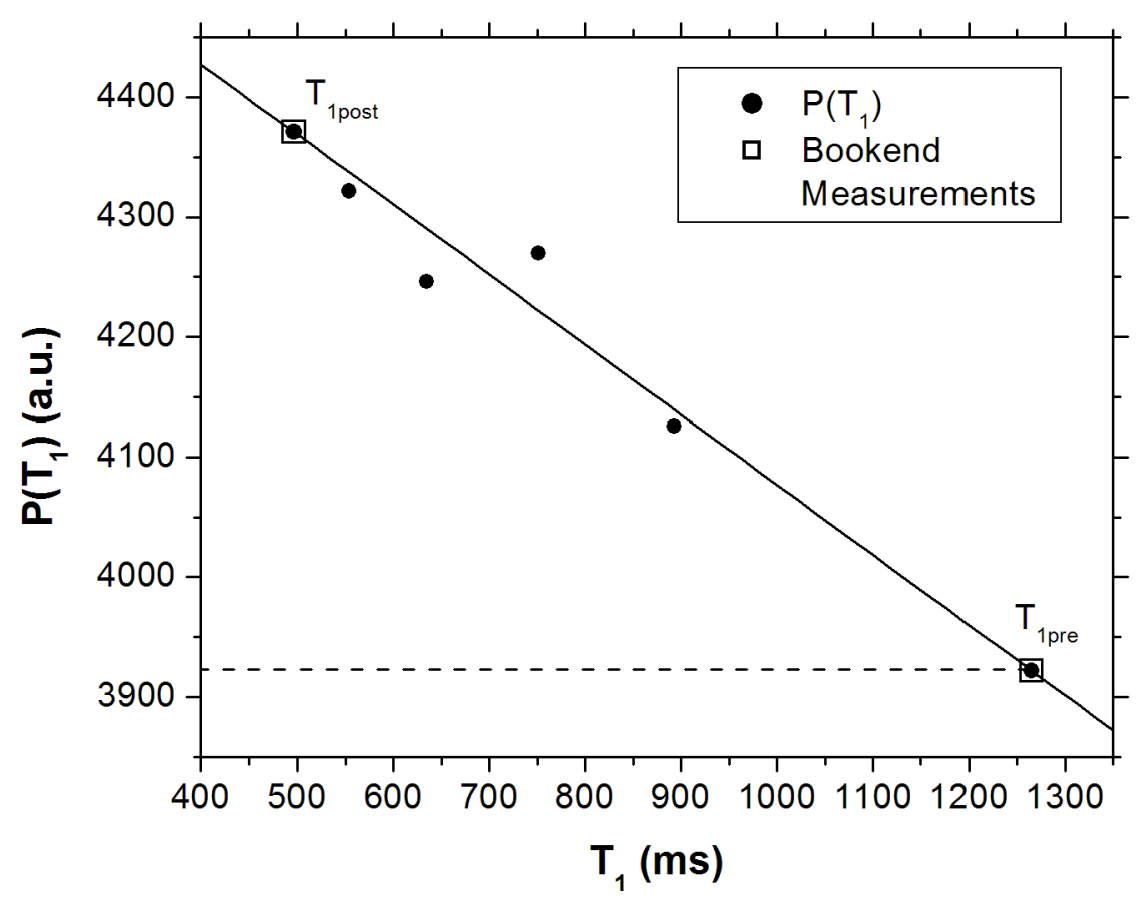

Figure 2.7: Experimentally determined $P\left(T_{1}\right)$ for several values of $T_{1}$ (dots). Squares represent the Bookend measurements of $P\left(T_{1}\right)$. The solid black line represents the linear approximation of $P$ used in the BE calibration. The dashed line represents $P$ as assumed by $T_{1 \text { pre }}$ calibration. 
This curve was generated from measurements made on the 3T Siemens MR scanner at the Ottawa Hospital using a phantom and following the experimental procedure presented in Sections 3.4.1 and 3.4.2. The values of $P\left(T_{1}\right)$ seen in Figure 2.7 were generated using MOLLI $T_{1}$ maps explained in greater detail in Section 3.3.3.

The point at the far right of Figure 2.7, which corresponds to the first point measured in a DCE experiment, has the highest $T_{1}$ because no contrast agent has been introduced yet. The point on the far left, which corresponds to the last point measured, corresponds to the plateau of $[G d]$ in the EES of a tumour. These two points have squares around them signifying that they correspond to the bookend measurements in this experiment. The other points are not typically measured during a DCE-MRI experiment. The solid line connecting the two bookend measurements is the linear fit assumed to approximate the relationship between $P\left(T_{1}\right)$ and $T_{1}$. As is evident from Figure 2.7, this approximation does a suitable job of estimating $P\left(T_{1}\right)$ overall.

In contrast, the dashed line in Figure 2.7 extending from the first $T_{1}$ measurement ( $\left.T_{1 \text { pre }}\right)$, represents the value of $P\left(T_{1}\right)$ for the $T_{1 \text { pre }}$ algorithm. It is used as the calibration factor and assumed to remain unchanged throughout the imaging experiment. It is clear that this method severely underestimates the value of $P\left(T_{1}\right)$, especially at low $T_{1}$ (high $[G d]$ ) - values that are commonly observed when a bolus of contrast agent is present. The effect of these signal calibrations on the FLASH signal strength can be seen in Figure 2.8. After calibration of Eq. (2.27), the ratio of the measured signal strength $S$ (left hand side of Eq. (2.27)) to the calibrated theoretical signal strength (right hand side of Eq. (2.27)) was taken. The resulting signal ratios were plotted against their corresponding $T_{1}$ for both the $T_{1 \text { pre }}$ and BE methods. It is evident from Figure 2.8 that the BE method is better suited to handle these variations in signal strength than the $T_{1 \text { pre }}$ method. BE constrains the signal strength equation and does not allow it to drift away from theory as a function of $T_{1}$. 


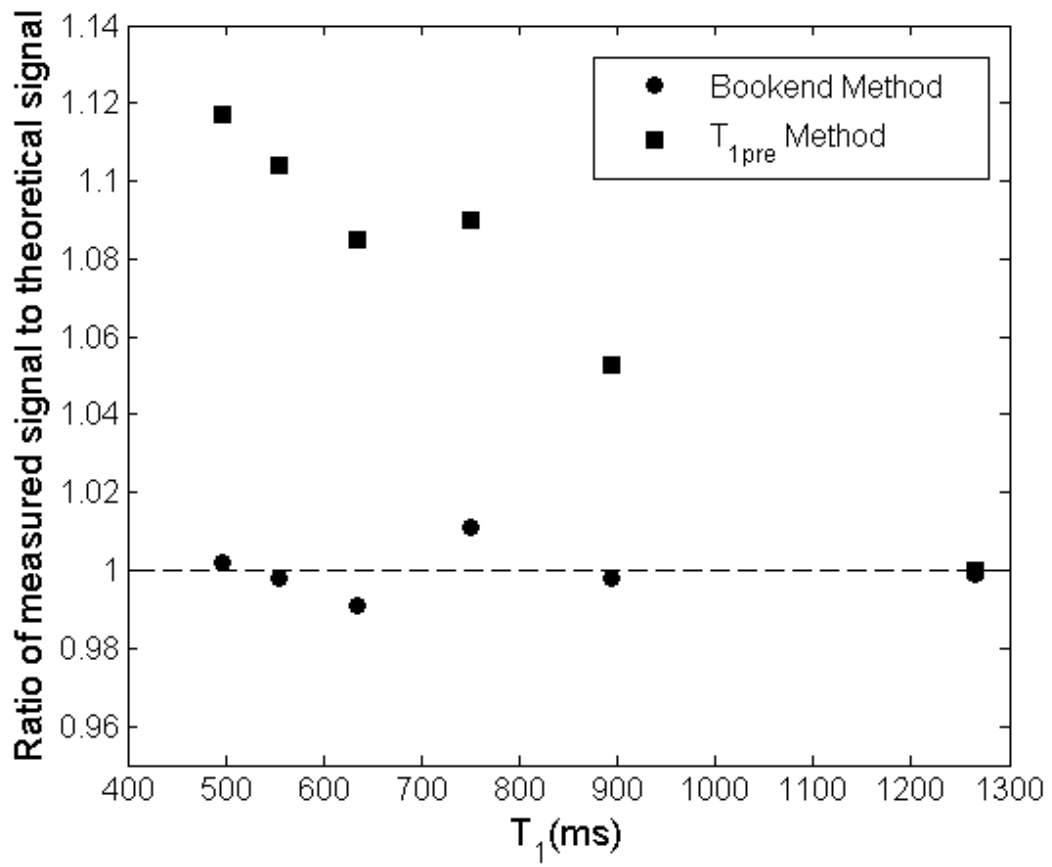

Figure 2.8: Comparison of measured FLASH signal to calibrated theoretical FLASH signal. Signal ratio is calculated as $S / P\left(T_{1}\right) F\left(\alpha, T R, T_{1}\right)$ from Eq. (2.27). A signal ratio closer to 1.00 represents a better calibration.

The use of the Bookend method to provide an overall correction factor to calibrate the FLASH signal strength equation is justified in the above. It can be seen that the signal strength may diverge from theory, with the degree of divergence being a function of $T_{1}$. These problems arise from many areas such as slice profile effects (in $2 \mathrm{D}$ acquisitions), RF miscalibration, $B_{1}$ inhomogeneity and others. The use of an all-encompassing calibration factor such as provided by the BE method is essential, if one wishes to use a FLASH-type sequence for accurate, repeatable, and uniform [Gd] quantification in DCE-MRI. The preceding statement will be proven by this work. 


\section{Chapter 3}

\section{Materials and Methods}

This chapter will detail how the experiments in this work were performed. The methods for acquiring the rapid DCE signal, $T_{1}$ mapping, and $[G d]$ quantification will be explained. The postprocessing and methods of image analysis will also be explained.

All experiments were conducted at The Ottawa Hospital Civic Campus, Ottawa, Ontario, Canada. The experiments were performed on a Siemens 3T Trio MR imaging system. All images were acquired using a Siemens 32-channel head coil. Postprocessing and image analysis were performed using MATLAB, IDL, and ImageJ.

All measurements were performed on a homemade MRI test phantom. No experiments with human subjects were conducted.

\subsection{The Phantom}

In this project, the ability of the $T_{1 p r e}$ and Bookend methods to accurately measure the concentration, $[G d]$, of a Gd-based MRI contrast agent from a rapidly measured $T_{1}$-weighted pulse sequence, was tested. In addition, there is interest in quantifying the spatial variation of $[G d]$ in the plane of individual images as well as in the thoughplane direction throughout a relatively large volume (mimicking the brain). To do 


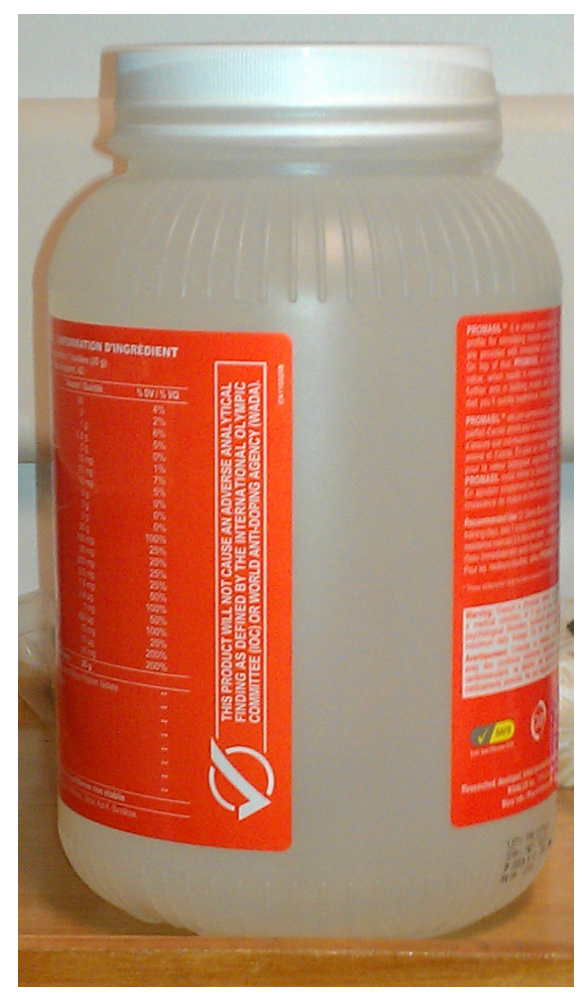

(a)

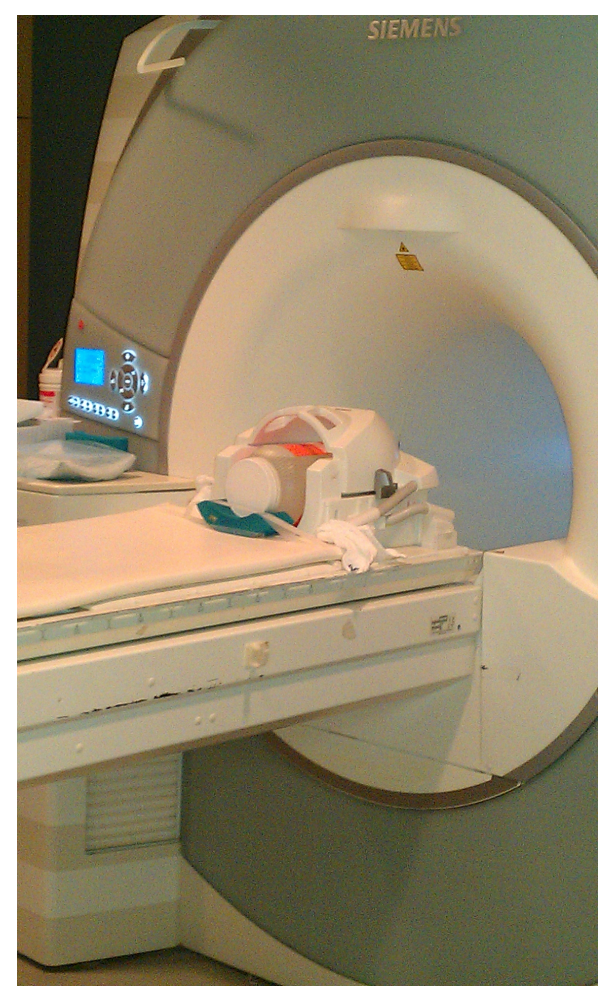

(b)

Figure 3.1: The phantom (a) and the phantom loaded into the head coil prior to translation into the magnet bore (b).

this, a phantom that had a large volume and whose contrast agent concentration could be easily manipulated, was required.

The phantom used in these experiments was an approximately 4 litre jug that was filled with water and doped with various amounts of Gadovist to manipulate $[G d]$ and $T_{1}$. Once doped to the desired concentration of contrast agent, the phantom was sealed and placed in the head coil for imaging. The phantom provided a large, uniform volume that could be used to determine the accuracy of $[G d]$ measurements. 


\subsection{DCE Signal Acquisition}

After injection of the contrast agent into the patient in an actual DCE-MRI procedure, the bolus travels through the bloodstream at a fairly quick pace. Therefore, to properly capture the evolution of $[G d]$ with time, an imaging sequence with high temporal resolution is required. Since $[G d]$ is related to a change in $T_{1}$, ideally the actual $T_{1}$ change would be accurately measured with high temporal resolution, then simply converted to $[G d]$ using Eq. (2.22). However, this is not practical since accurate $T_{1}$ mapping tends to be an inherently lengthy process. Instead, a faster $T_{1}$-weighted pulse sequence is used as an intermediate step $[35,36]$. The raw signal enhancement due to the presence of the contrast agent is tracked rapidly in time, then this information is used to obtain information about the $T_{1}$ change and, in turn, $[G d]$. As mentioned previously, a common pulse sequence chosen for this role is a spoiled gradient echo-type sequence, due to their short acquisition time. A Siemens 3D-FLASH pulse sequence was used in this work.

When a system of spins is rapidly and repeatedly excited by a series of successive RF pulses where, $T R \ll T_{2}, T_{1}$, the longitudinal magnetization will reach a state of dynamic equilibrium known as the "steady-state" [37]. What this essentially means is that after a certain number of RF pulses, the longitudinal magnetization after the next pulse will relax back to its initial value prior to the pulse, which is less than the full static equilibrium value. So, the same amount of longitudinal magnetization is tipped into the transverse plane every repetition without the need to wait several $T_{1}$ 's for full static equilibrium to be achieved. In addition, since the repetition time is so fast, complete $T_{2}$ decay will not have time to occur. Thus, prior to the next pulse, there will be residual magnetization in the transverse plane. In the case of FLASH, this magnetization is destroyed through the use of spoiler gradients and RF spoiling which dephases any residual magnetization after spatial localization/acquisition and 


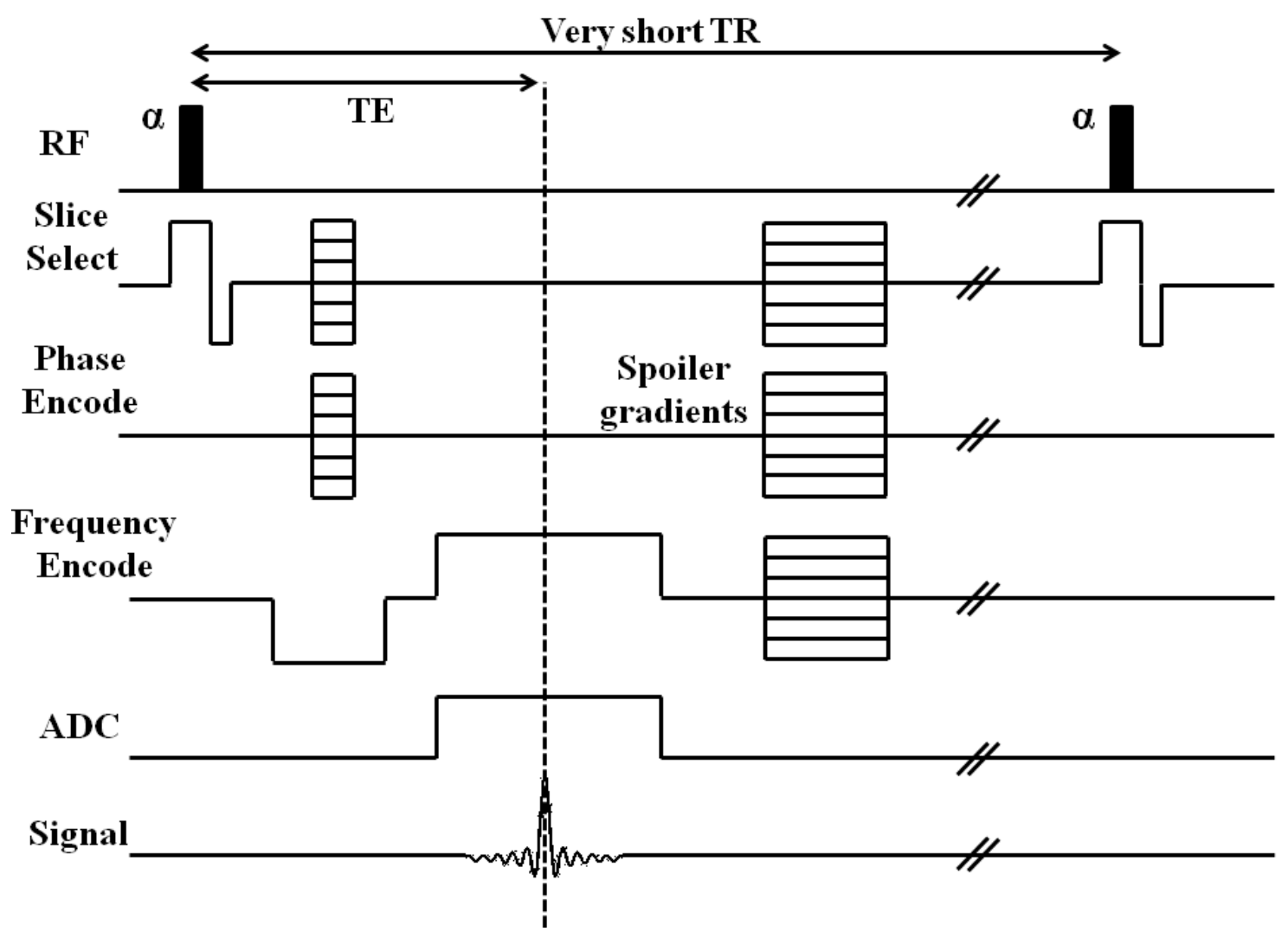

Figure 3.2: Pulse sequence diagram for a 3D-FLASH acquisition. 
prior to the next RF pulse $[38,39]$. This is done so magnetization from one excitation does not contaminate the signal of successive excitations. The combination of longitudinal steady state imaging and spoiling allows for extremely short repetition times, vastly increasing image acquisition speed. This allows FLASH to have good temporal resolution and makes it a prime candidate for DCE-MRI.

Under perfect spoiling conditions where $M_{x y}=0$ prior to the next excitation, the uncalibrated FLASH signal strength equation is similar to Eq. (2.27):

$$
S=M_{0} \sin \alpha \frac{1-e^{-T R / T_{1}}}{1-e^{-T R / T_{1}} \cos \alpha} e^{-T E / T_{2}^{*}},
$$

where $M_{0}$ is the equilibrium magnetization. Once again, typically $T E \ll T_{2}^{*}$ so that $T_{2}^{*}$ effects can be ignored. By using a short $T R$ and a moderate $\alpha$, the signal will be $T_{1}$-weighted. The presence of contrast agent causes $T_{1}$ to decrease and the signal in the image to increase. Figure 3.3 illustrates an example of a contrast agent injection. The signal enhancement due to the presence of contrast agent is what is ultimately used to determine $[G d]$. 


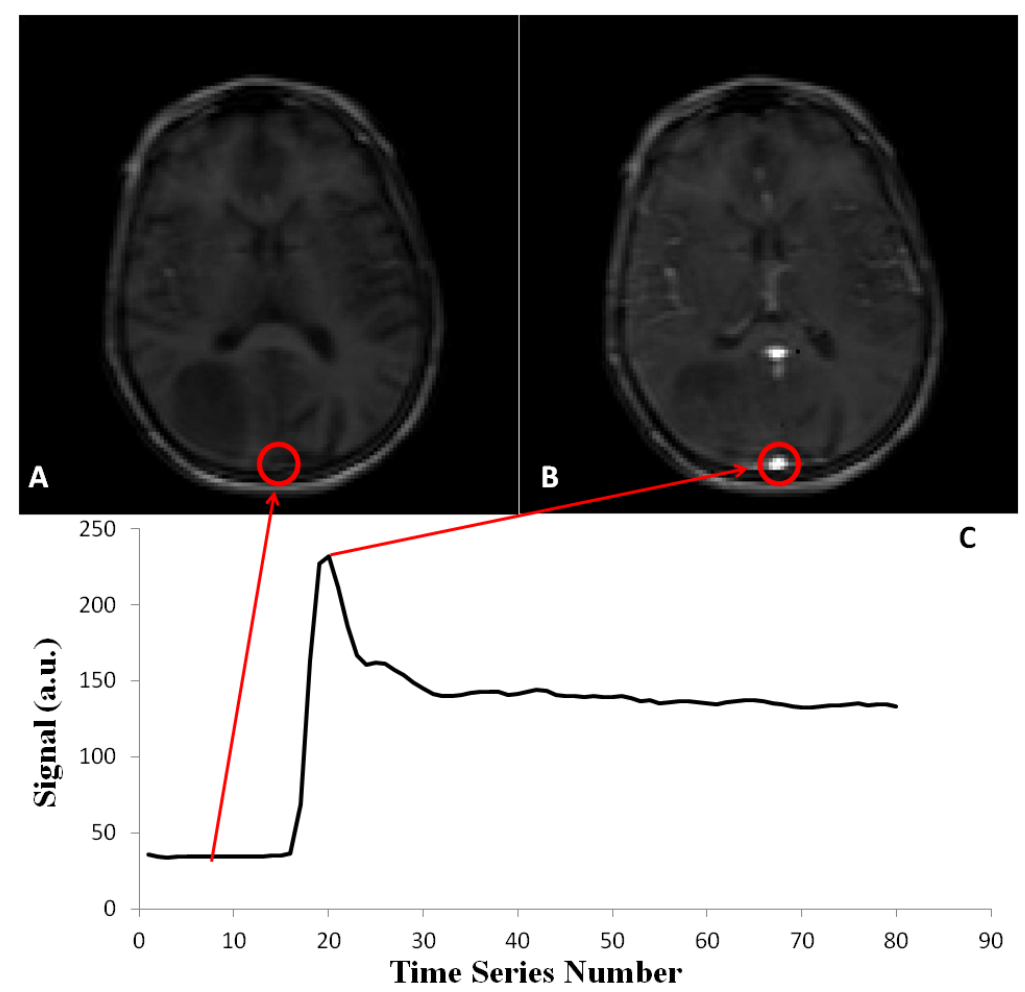

Figure 3.3: Axial images of the brain before the arrival of the bolus of contrast agent $(A)$ and during the peak of signal enhancement due to presence of contrast agent (B). The signal enhancement curve of the area outlined by the circle (C). Arrows indicate the position along the signal enhancement curve for both images. 
The clinical DCE-MRI protocol used at The Ottawa Hospital uses a 3D-FLASH sequence with the pulse sequence parameters given in Table 3.1. The temporal resolution given in Table 3.1 is for a full volume acquisition. The 3D-FLASH sequence

\begin{tabular}{cc} 
Parameter & Value \\
\hline \hline Flip Angle & 30 degrees \\
TR & $6.5 \mathrm{~ms}$ \\
TE & $1.65 \mathrm{~ms}$ \\
Field of View (FOV) & $220 \times 220 \mathrm{~mm}^{2}$ \\
Slice Thickness & $5 \mathrm{~mm}$ \\
Spatial Resolution & $1.72 \times 1.72 \mathrm{~mm}^{2}$ \\
Number of Slices & 18 \\
Temporal Resolution & $3.52 \mathrm{~s}$ \\
Number of Timepoints & 80 \\
Matrix Size & $128 \times 128$ \\
Phase Encode Steps & 86 \\
\hline \hline
\end{tabular}

Table 3.1: Clinical 3D-FLASH pulse sequence parameters.

used in this project was edited to have to same parameter settings as presented in Table 3.1.

\section{3 $\quad T_{1}$ Quantification}

Accurately measuring $T_{1}$ is critical for any DCE-MRI experiment. In this project $T_{1}$ was measured using a variety of $T_{1}$ mapping techniques. Excluding the reference technique, all $T_{1}$ mapping methods had several criteria that must be met regarding clinical practicality and usefulness. Firstly, the method needed to have a reasonably short acquisition time (under 3 minutes). Secondly, the geometry and spatial resolution of the image had to exactly match that of the 3D-FLASH DCE acquisition, 


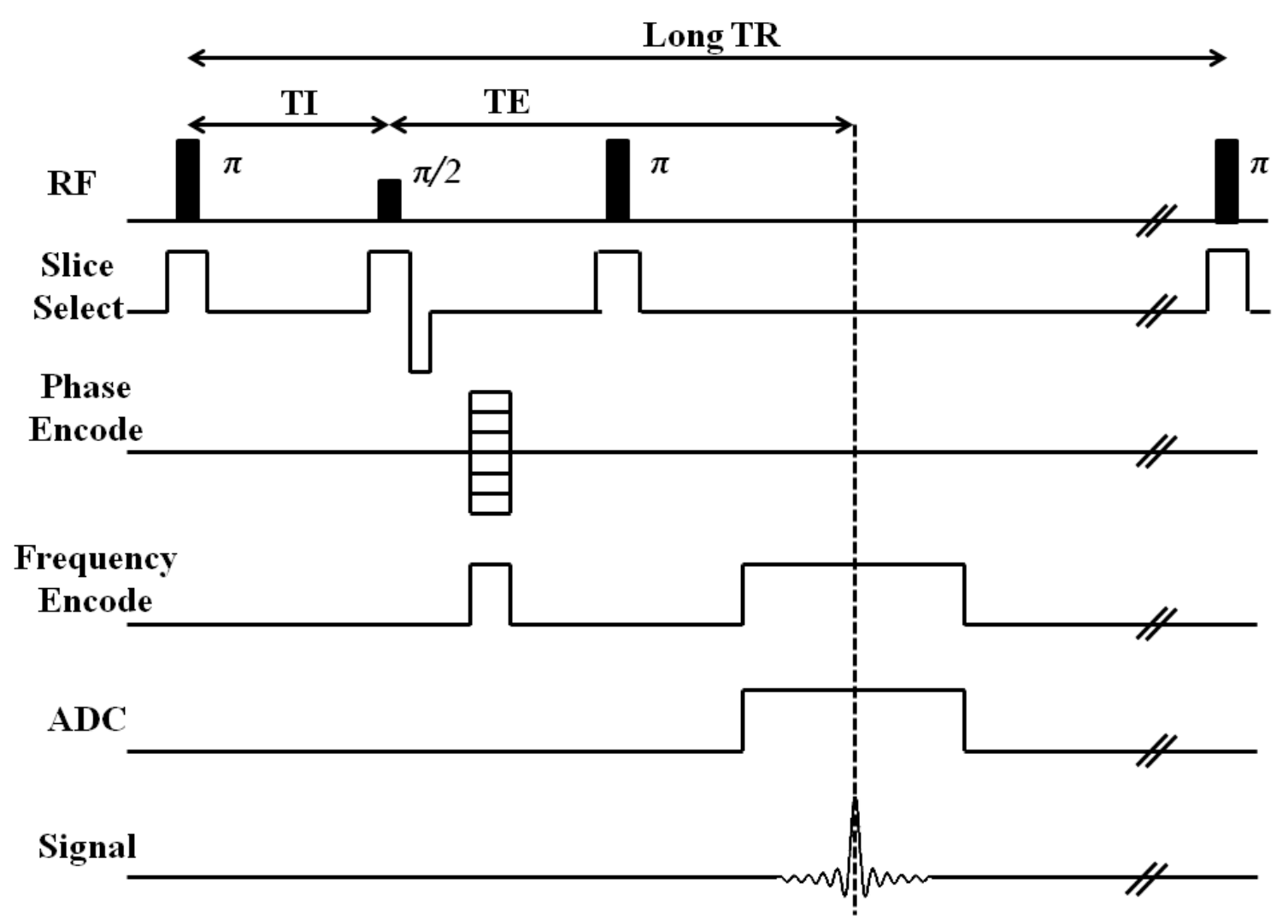

Figure 3.4: Pulse sequence diagram for the traditional inversion recovery sequence.

given in Table 3.1, in order to allow parametric voxel by voxel analysis. The following section describes these $T_{1}$ mapping methods in addition to the reference technique.

\subsubsection{Gold Standard: Traditional Inversion Recovery}

One of the oldest [40], and most commonly used [41,42], methods for quantifying $T_{1}$ in MRI is known as the Inversion Recovery (IR) method. IR is so named because it directly samples the relaxation corresponding to $M_{z}$ regrowth as a function of time after an inversion or $\pi$ RF pulse. The pulse sequence diagram for a typical spin echo inversion recovery sequence is shown in Figure 3.4.

The time between the initial inversion pulse and the measurement $\pi / 2$ pulse is known as the inversion time $(T I)$, as indicated in Figure 3.4. The signal strength 
equation for a pulse sequence of this type [43]

$$
S=K\left(1-2 e^{-T I / T_{1}}+e^{-T R / T_{1}}\right) e^{-T E / T_{2}}
$$

Typically, the $T R$ is set to be several $T_{1}$ 's in length in order to allow full recovery of the magnetization back to equilibrium before each $\pi$ pulse. Therefore $T R \gg$ $T_{1}$. Conversely, the $T E$ is made to be short such that $T E \ll T_{2}$. The resulting simplification of Eq. (3.2) is then

$$
S=A-B e^{-T I / T_{1}}
$$

where the single constant $K$ has been replaced by $A$ and $B$ to take into account the possibility of imperfect inversion pulses. $T_{1}$ may then be quantified by measuring the signal strength, $S$, while varying $T I$ such that several points along the relaxation curve are sampled. It is relatively straightforward to estimate $T_{1}$. A three-parameter, non-linear, Levenberg-Marquardt algorithm was used to extract $T_{1}$ in MATLAB in a parametric voxel by voxel manner from the signal intensity images.

IR is the favoured way to quantify $T_{1}$ because it is a direct measurement of the relaxation curve and is insensitive to $B_{1}$ inhomogeneities. It is for this reason that IR was used as a "gold standard" to which all other $T_{1}$ measurements conducted within the scope of this project were compared. In this project, a Siemens 2D Turbo Spin Echo (TSE) IR pulse sequence was used. TSE-IR benefits from decreased acquisition time due to the collection of multiple spin echoes per inversion pulse (referred to as the echo train). The relevant pulse sequence parameters are given in Table 3.2. The relaxation curve was sampled at the following timepoints: 500ms, 750ms, 1000ms, $1500 \mathrm{~ms}$, 2000ms, and $2690 \mathrm{~ms}$. A single, axial slice at the centre of the phantom was imaged. This slice approximately corresponded with the centre of the imaging volume 
in the 3D-FLASH acquisition. Unfortunately, full volume acquisition using TSE-IR is still far too lengthly to be implemented in the clinic, especially since the acquisition must be repeated for multiple TI's in order to properly sample the relaxation curve.

\begin{tabular}{cc} 
Parameter & Value \\
\hline \hline TR & $6500 \mathrm{~ms}$ \\
TE & $12 \mathrm{~ms}$ \\
Field of View (FOV) & $250 \times 250 \mathrm{~mm}^{2}$ \\
Slice Thickness & $10 \mathrm{~mm}$ \\
Spatial Resolution & $1.95 \times 1.95 \mathrm{~mm}^{2}$ \\
Matrix Size & $128 \times 128$ \\
Echo Train Length & 7 \\
\hline \hline
\end{tabular}

Table 3.2: TSE-IR pulse sequence parameters.

\subsubsection{The Variable Flip Angle Method}

A technique commonly used to measure $T_{1}$ clinically is known as the Variable Flip Angle (VFA) method. VFA is a FLASH-based technique and is very different from inversion recovery. Unlike IR, FLASH-based sequences make use of longitudinal steadystate magnetization and are not required to return to full static equilibrium prior to the next RF preparation pulse. As previously explained, this allows for a very short $T R$, greatly reducing acquisition time. The signal strength equation for a FLASH sequence is given in Eq. (3.1). Since the signal is a function of $\alpha$, we may exploit this dependence to estimate $T_{1}$ by measuring the signal strength, $S$, as $\alpha$ is varied, while holding all other parameters constant [44,45]. After rearrangement and linearization of Eq. (3.1), the following is linear equation is obtained

$$
\frac{S}{\sin \alpha}=\frac{S}{\tan \alpha} e^{-T R / T_{1}}+M_{0}\left(1-e^{-T R / T_{1}}\right)
$$


where $S / \sin \alpha$ and $S / \tan \alpha$ represent the $y$ and $x$ data to be fitted. It is clear that the slope of Eq. (3.4) is $m=e^{-T R / T_{1}}$ and by simple rearrangement

$$
T_{1}=-\frac{T R}{\ln (m)}
$$

In the two flip angle limit, there is an analytical method to solve for $T_{1}[46,47]$. This is the method that is used in the clinical Siemens VFA $T_{1}$ mapping protocol. If two raw signal images are obtained using two different flip angles while holding all other parameters constant, the ratio of the signals is given as $\rho=S_{\psi} / S_{\phi}$, where $\psi$ and $\phi$ are the flip angles. $T_{1}$ may then be extracted according to

$$
T_{1}=\frac{T R}{\ln [f(\rho, \psi, \phi)]},
$$

where

$$
f(\rho, \psi, \phi)=\frac{\rho \sin \psi \cos \phi-\sin \phi \cos \psi}{\rho \sin \psi-\cos \phi} .
$$

Because of the very short $T R$, VFA is capable of extremely quick $3 \mathrm{D}$ acquisitions making it an excellent candidate for clinical $T_{1}$ quantification, as is required in DCEMRI. The Siemens VFA protocol takes approximately 30 seconds to acquire a $T_{1}$ map with the geometry and spatial resolution specified in Table 3.1.

Unfortunately, the VFA method suffers from some serious limitations. In particular, VFA is sensitive to inhomogeneities in the RF transmit field $\left(B_{1}\right)$ which may cause spatial variations in signal intensity $[48,49]$. Recall from Eq. (2.13) that the shape of the $B_{1}$ pulse envelope with time and the pulse duration determine the flip angle, $\alpha$. If the $B_{1}$ field varies spatially, this may cause spatial variation of $\alpha$. In the VFA method, the value of $\alpha$ is assumed to be constant throughout the FOV. Therefore, errors in $T_{1}$ mapping may arise.

One of the most common ways to mitigate errors introduced by $B_{1}$ inhomogeneity 


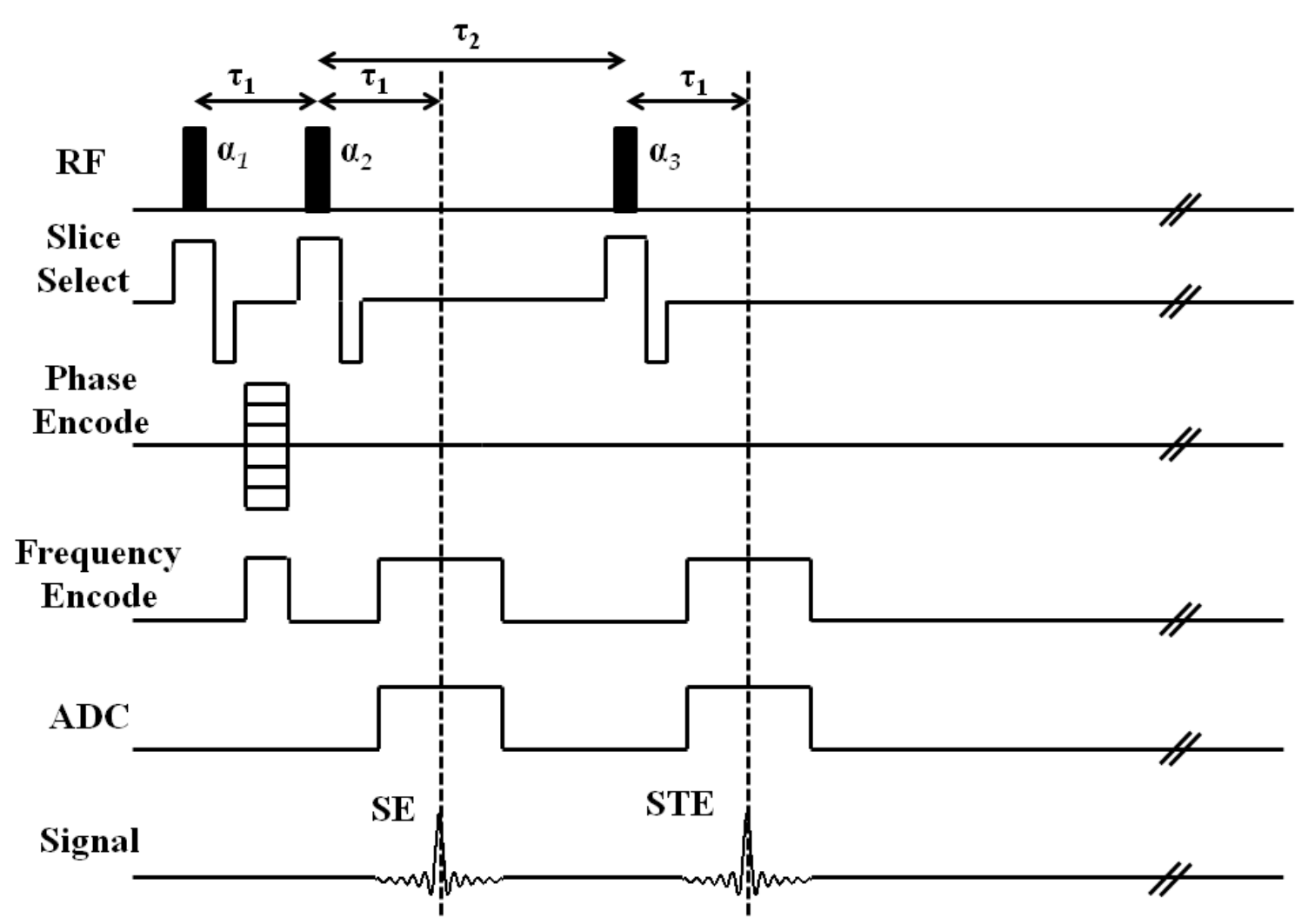

Figure 3.5: Pulse sequence diagram indicating the generation of a stimulated echo.

is to "map" the effect of the $B_{1}$ field. $B_{1}$ mapping produces a parametric image of the spatial distribution of the delivered flip angle within the FOV. There are many different $B_{1}$ mapping methods found in the literature such as Bloch-Siegert [50], Actual Flip angle Imaging (AFI) [51], and others. There are also methods that attempt to accurately measure $T_{1}$ in the presence of $B_{1}$ inhomogeneity without specifically relying on $B_{1}$ mapping [52-54]. In this project, a Siemens works-inprogress (WIP) package incorporating $B_{1}$ correction to VFA $T_{1}$ maps was used. The WIP uses the well known stimulated echo $B_{1}$ mapping method [55] to determine spatial flip angle variation. Stimulated echoes (STE) are a type of echo that are created when three RF pulses are applied in succession [56,57] as shown in Figure 3.5. In this method, the ratio of the signal intensities between the primary echo (SE) and 
the STE are used to estimate the flip angle. The corresponding signal intensities are given as

$$
S_{S E}=K \sin \alpha_{1} \sin ^{2}\left(\alpha_{2} / 2\right) e^{-2 \tau_{1} / T_{2}}
$$

and

$$
S_{S T E}=\frac{K}{2} \sin \alpha_{1} \sin \alpha_{2} \sin \alpha_{3} e^{-\tau_{2} / T_{1}} e^{-2 \tau_{2} / T_{2}}
$$

Now for this particular $B_{1}$ mapping STE sequence, let $\alpha_{1}=\alpha_{3}=\alpha$ and $\alpha_{2}=2 \alpha$. The ratio of Eq. (3.9) to Eq. (3.8) then reduces to

$$
\frac{S_{S T E}}{S_{S E}}=\frac{1}{2} \frac{\sin 2 \alpha}{\sin \alpha} e^{-\tau_{2} / T_{1}}
$$

Using a simple trigonometric substitution and assuming $\tau_{2} \ll T_{1}$, Eq. (3.10) reduces to

$$
\frac{S_{S T E}}{S_{S E}}=\cos \alpha
$$

and the actual flip angle delivered is

$$
\alpha=\arccos \left[\frac{S_{S T E}}{S_{S E}}\right] .
$$

A parametric, relative $B_{1}$ factor map may then be generated from

$$
\kappa=\frac{\alpha_{\text {actual }}}{\alpha_{\text {nominal }}}
$$

where $\alpha_{\text {nominal }}$ represents the programmed flip angle and $\alpha_{\text {actual }}$ represents the true flip angle as measured during the $B_{1}$ mapping. $\kappa$ may then be used to correct Eq. (3.7) by correcting the flip angles:

$$
f_{c}(\rho, \psi, \phi)=\frac{\rho \sin \kappa \psi \cos \kappa \phi-\sin \kappa \phi \cos \kappa \psi}{\rho \sin \kappa \psi-\cos \kappa \phi} .
$$




\begin{tabular}{cc} 
Parameter & Value \\
\hline \hline TR & $20 \mathrm{~ms}$ \\
TE & $1.23 \mathrm{~ms}$ \\
$\phi$ & 4 degrees \\
$\psi$ & 25 degrees \\
\hline \hline
\end{tabular}

Table 3.3: VFA pulse sequence parameters.

In this work, both uncorrected VFA (uVFA) and corrected VFA (cVFA) $T_{1}$ maps were used. The geometry and spatial resolution matched that of the FLASH DCE acquisition in Table 3.1. The pulse sequence parameters specific to VFA are given in Table 3.3. The $B_{1}$ mapping sequence takes about 30 seconds. Therefore, to implement cVFA instead of uVFA, $T_{1}$ mapping time is increased from 30 seconds to 1 minute per map.

\subsubsection{Modified Look-Locker Inversion Recovery}

In 1970, D.C. Look and D.R. Locker published an innovative $T_{1}$ measurement technique for NMR spectroscopy [58] that has since been readily applied to $T_{1}$ mapping in MRI with success [59,60]. Typically known as the "Look-Locker" (LL) method, this technique works by sampling the longitudinal relaxation curve many times after a single inversion preparatory pulse. This is done by perturbing the longitudinal magnetization repeatedly with small RF pulses as it relaxes to equilibrium. The signal strength equation for a method of this type using an inversion preparatory pulse is given by $[61,62]$

$$
S=A-B e^{-T I / T_{1 e f f}}
$$


where the magnetization relaxes with an effective $T_{1}$ given by [61]

$$
T_{1 e f f}=\frac{T_{1}}{(B / A-1)}
$$

As with traditional IR, a three parameter curve fit is performed to fit the data to the perturbed relaxation curve to extract $T_{1 e f f}$. The true $T_{1}$ may then be calculated by rearranging Eq. (3.16).

This type of $T_{1}$ mapping technique is advantageous because it samples the entire relaxation curve after a single inversion, increasing the overall acquisition speed. Further acquisition improvements may be made by coupling the LL technique with a fast readout method. In this work, a Siemens WIP which was based on the Modified Look Locker Inversion recovery (MOLLI) sequence [63] was used. MOLLI was originally designed to be an ECG triggered cardiac sequence that samples the relaxation curve in three sweeps. This project (as well as in vivo neuro studies) will not require triggering and only a single sweep through the relaxation curve is necessary.

The readout section of this pulse sequence also benefits from fast acquisition through the use of steady-state imaging. However, unlike FLASH, this sequence does not spoil the residual transverse magnetization, instead refocusing it prior to each pulse. This family of acquisitions is known as Steady-State Free precession (SSFP). The particular type of acquisition used in the MOLLI WIP is known as True Fast Imaging with Steady State Precession (trueFISP) [64]. In this type of acquisition, the transverse magnetization is fully refocused and centered in time with the next RF pulse such that residual magnetization contributes to the measured signal without affecting the $T_{1}$-weighting. The entire MOLLI pulse sequence is shown in Figure 3.6. The parameter $\tau$ is the time interval between successive RF pulses, and is a programmed sequence parameter.

An axial, 2D trueFISP readout was used with a non-selective inversion preparation 


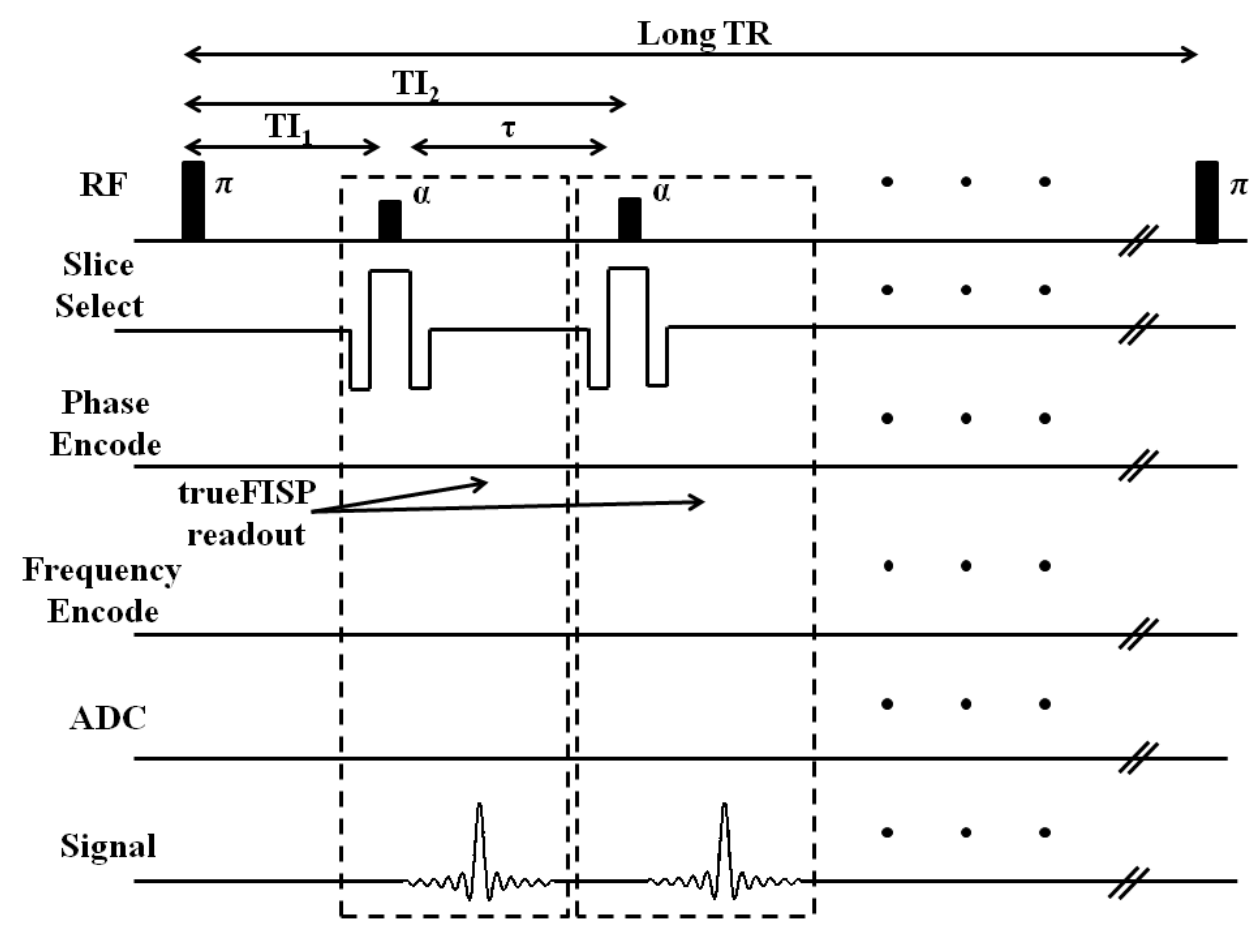

(a) Overall Look-Locker pulse sequence with trueFISP readout indicated in dashed boxes.

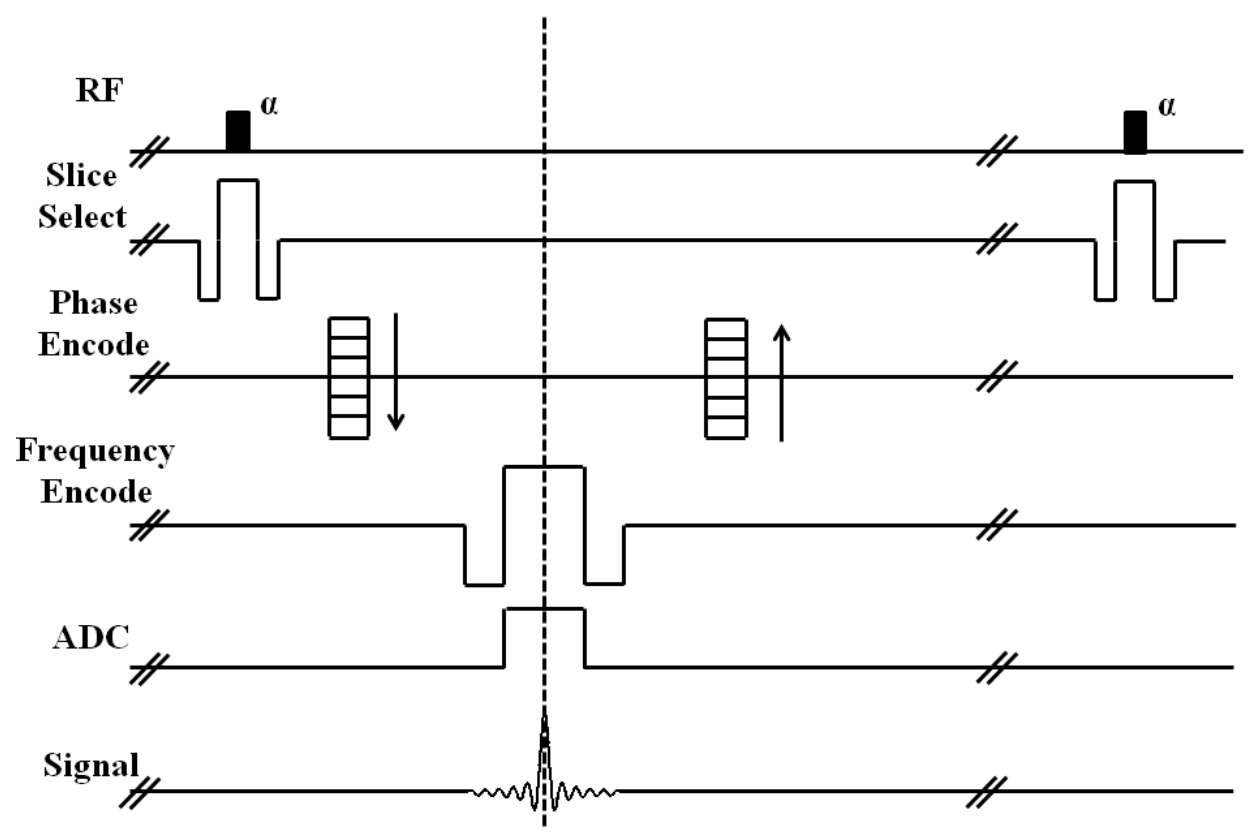

(b) Detailed view of the trueFISP readout.

Figure 3.6: Overview of the entire MOLLI pulse sequence. 


\begin{tabular}{cc} 
Parameter & Value \\
\hline \hline TR & $6085 \mathrm{~ms}$ \\
TE & $1.08 \mathrm{~ms}$ \\
Flip Angle & 30 degrees \\
$\tau$ & $750 \mathrm{~ms}$ \\
Initial TI & $85 \mathrm{~ms}$ \\
\hline \hline
\end{tabular}

Table 3.4: MOLLI pulse sequence parameters.

pulse. As always, the geometry and spatial resolution matched those used in the DCE acquisition given in Table 3.1. The pulse sequence parameters specific to MOLLI are given in Table 3.4. The timepoints sampled along the curve were: 85ms, 835ms, 1585ms, 2335ms, 3085ms, 3835ms, 4585ms, and 5335ms. The MOLLI sequence takes approximately 2 minutes to acquire a $T_{1}$ map. Therefore, more time is sacrificed in the interest of $T_{1}$ mapping.

\subsection{Phantom Experiments}

\subsubsection{Measurements}

The major purpose of this project is to evaluate and quantify the ability of the $T_{1 \text { pre }}$ and Bookend methods to measure the concentration of contrast agent within a volume. This was done using a homemade phantom. To begin, the phantom was filled with water and doped with approximately $0.5 \mathrm{~mL}$ of Gadovist to lower the $T_{1}$ from approximately $4000 \mathrm{~ms}$, that of bulk water, to around $1200-1400 \mathrm{~ms}$. This was done to establish a baseline state that somewhat mimics the natural $T_{1}$ environment of the brain (i.e. brain tissue without contrast agent present). Literature values report that the $T_{1}$ of white and gray matter are approximately $830 \mathrm{~ms}$ and $1330 \mathrm{~ms}$, respectively, at $3 \mathrm{~T}$ [65]. By beginning the experiment in the higher end of this $T_{1}$ spectrum, the 
range of $T_{1}$ 's observed in the brain will be covered.

Once the phantom is doped to this initial $T_{1}$, this will be considered the zero concentration mark, mimicking the brain before contrast agent injection. The water in the phantom is stirred to distribute the contrast agent as uniformly as possible. The phantom is then sealed, placed into the head coil, and translated into the magnet bore in preparation for imaging. The churning of the water due to stirring and movement of the patient table as it translates may introduce streaking and motion artifacts into the images. Therefore, it was necessary, prior to any imaging, for phantom to stay in the magnet bore for a period of time in order to allow the water to settle so that these artifacts would not occur. It was determined that a period of 15 minutes wait time was sufficient in allowing the water to settle. Before each imaging run, this was verified through the use of localizers (very quick, single slice scans).

Once the wait period had elapsed, imaging of the phantom was performed. Each imaging run consisted of all the imaging procedures previously discussed: TSE-IR, VFA, STE- $B_{1}$, MOLLI, and FLASH DCE. The workflow of this experiment is shown in Figure 3.7. Once this imaging experiment was completed, the phantom was removed from the bore and another dose of contrast agent was added to increase the concentration. This process was repeated many times until measurements for all concentrations were completed. For each concentration, accurate knowledge of $T_{1}$, and therefore, $[G d]$, was obtained from the TSE-IR results.

The range of concentrations covered from the initial baseline concentration was $0 \mathrm{mM}$ to approximately $0.3 \mathrm{mM}$. This range was chosen because it reflects the range of concentrations typically seen in vivo. The Gadovist, which comes in vials at a concentration of $1 \mathrm{mmol} / \mathrm{mL}$, was diluted by a factor of 10 with water to allow for easier quantification using a 1mL syringe. The experiments either had 10 or 6 successive concentrations depending on time constraints during imaging. This experiment was then repeated several times on different days in order to evaluate the reproducibility 


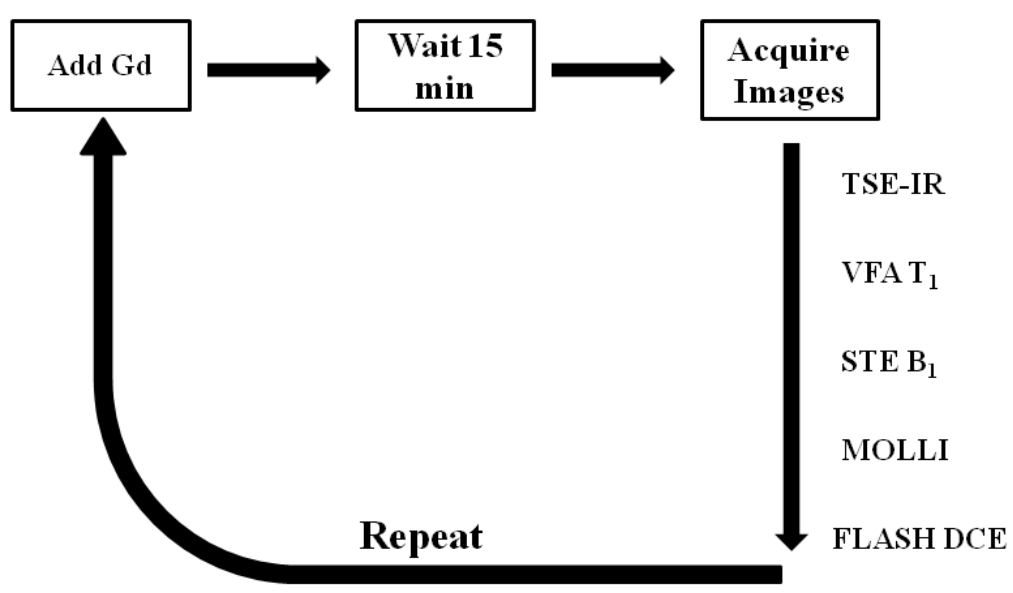

Figure 3.7: Workflow of the $[G d]$ measurement experiment. The experiments begin at the top left.

of each procedure over time.

\subsubsection{Postprocessing and Image Analysis}

Once the image data was acquired, it was analyzed in the following ways. The TSE-IR $T_{1}$ maps were used as a measurement of the true $T_{1}$ in the phantom. From here, the reference concentration maps were then calculated using Eq. (2.22) on a voxel by voxel basis, with the initial $T_{1}$ map designated to be the pre-injection $T_{1}$ map, $T_{1 \text { pre }}$. The VFA $T_{1}$, STE- $B_{1}$, and MOLLI measurements were used to evaluate the calibration factor, $P\left(T_{1}\right)$ introduced in Section 2.3. The 3D FLASH signal strengths acquired at each concentration were then stacked in the $4^{\text {th }}$ dimension to mimic a DCE time series and produce a pseudo signal enhancement curve. In house code was used on all acquired images to perform rigid body motion correction. This removes any change in position that may have occurred while removing and replacing the phantom. The 3D-FLASH signal data were then used to determine $[G d]$ using both the $T_{1 \text { pre }}$ and the Bookend methods. These data were then analyzed a number of ways depending on the combination of $[G d]$ measurement procedure and $T_{1}$ mapping technique used, yielding a total of six unique methods for determining $[G d]$. Figure 3.8 illustrates the 


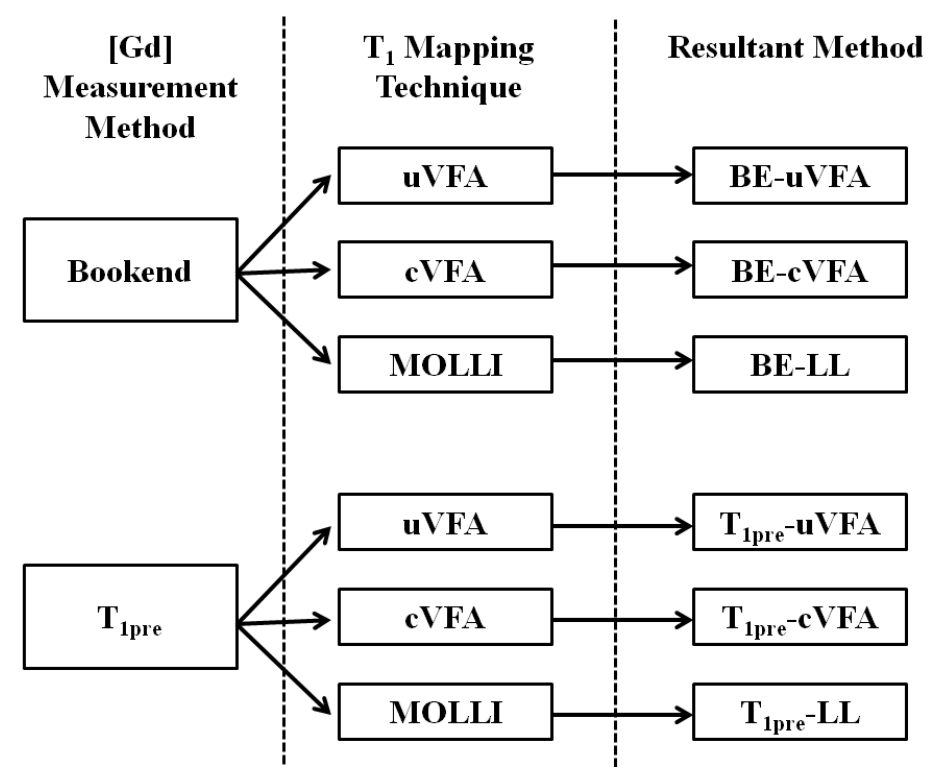

Figure 3.8: Diagram illustrating the different methods of $[G d]$ analysis.

formation of these methods. By analyzing these six methods, the effects of both the $[G d]$ quantification methods and $T_{1}$ mapping were evaluated and compared.

The methods outlined in Figure 3.8 were used to produce concentration maps with the same geometry and resolution as the original 3D-FLASH images indicated in Table 3.1. To analyze the results, an approximately $140 \mathrm{~cm}^{2}$ region of interest (ROI) was placed on the central $\left(9^{t h}\right)$ slice of the phantom. This slice was chosen because it is in the middle of the imaging volume and will not suffer from the slabprofile effects caused by imperfect excitation of the imaging volume near the outer edges in 3D acquisitions. This is clinically relevant because in a DCE-MRI procedure, the tumour or tissue of interest will be placed at the centre of the imaging volume. The values within the ROI were then used to calculate a mean concentration, used to assess accuracy, and a standard deviation, reflecting precision or uniformity. This was repeated for each concentration.

The results of each trial were then plotted against the reference concentrations determined from TSE-IR. The mean values are represented by the points themselves while the standard deviation was represented by the positive and negative vertical 
error bars. As a measure of accuracy of the resulting trendline, a linear regression was performed and the trends are assumed to begin at 0mM. The slope of said trend was used as a figure of merit for the $[G d]$ quantification method under consideration. Perfection is represented by a slope of 1.00 i.e. the measured concentrations for the method match exactly the reference concentrations. The minimum and maximum trends, formed by plotting the values illustrated by the negative and positive error bars, respectively, against reference values, were also analyzed by linear regression to give an overall representation of the inhomogeneity of each technique.

The through plane i.e. slice to slice comparison, was performed by measuring the mean and standard deviation values within the ROI propagated through all slices. These values were then referenced back to the $9^{t h}$ slice to give an overall representation of the interslice uniformity. This analysis was performed on the set of data collected on March 31, 2013. In this set of data, all $T_{1}$ mapping techniques were tested in conjunction with the same 3D-FLASH signal data in order to measure $[G d]$. This eliminates any variations in interslice homogeneity due to differences in the acquired DCE signal. The results were analyzed for a single reference concentration of $0.146 \mathrm{mM}$. This concentration was chosen because it is typical of $[G d]$ values encountered in tumours in vivo. 


\section{Chapter 4}

\section{Results}

The following section is dedicated to the presentation of the raw results of the experiments described in Chapter 3. It will begin with a brief look at the results of the reference technique. The results of all candidate clinical $T_{1}$ mapping techniques will be presented next, in order to illustrate the overall trend in $T_{1}$ accuracy. Finally and most significantly, the results of the $[G d]$ measurements using both the Bookend and $T_{1 \text { pre }}$ methods coupled with the different $T_{1}$ measurement techniques, will be shown.

\subsection{Reference Values}

Recall that in Section 3.3.1 it was explained that TSE-IR was to be used as the gold standard $T_{1}$ reference. In this work, all subsequent $T_{1}$ mapping was compared to the results of TSE-IR. Section 3.4.2 explains that the TSE-IR $T_{1}$ maps were used to calculate reference $[G d]$ maps on a voxel by voxel basis using Eq. (2.22).

The resulting $T_{1}$ maps measured with TSE-IR were highly uniform. The $T_{1}$ maps were analyzed and the average standard deviation over a $150 \mathrm{~cm}^{2}$ ROI across all maps was observed to be $5.2 \mathrm{~ms}$. This corresponds to an average standard deviation of $0.002 \mathrm{mM}$ in $[G d]$. There was no discernible trend in precision in either the $T_{1}$ maps or the $[G d]$ maps with increasing $[G d]$ (decreasing $T_{1}$ ). There was also no discernible 
change in behaviour from slice to slice. The reference $T_{1}$ and $[G d]$ values determined using the TSE-IR method are, therefore, considered to be accurate to within $5.2 \mathrm{~ms}$ and $2 \mu \mathrm{M}$, respectively.

\subsection{Results of $T_{1}$ Quantification}

Measuring $T_{1}$ is an important step in the DCE-MRI process. Therefore, it is paramount that the resulting $T_{1}$ maps be both as accurate and precise as possible, while adhering to clinical time acquisition concerns. Several different $T_{1}$ quantification techniques were considered and their performance was investigated. All $T_{1}$ measuring sequences were compared to traditional TSE-IR $T_{1}$ maps for determination of overall accuracy and precision. For each method, three $T_{1}$ values were looked at, ranging from high to intermediate to low. The results shown below have great impact on the $[G d]$ images reported later in this chapter.

\subsubsection{Variable Flip Angle Results}

As explained in Section 3.3.2, the results of variable flip angle $T_{1}$ mapping may be subcategorized into two groups: uncorrected and corrected. Uncorrected VFA (uVFA) maps are the raw $T_{1}$ maps delivered by the scanner without taking potential $B_{1}$ inhomogeneities into account. Conversely, corrected VFA (cVFA) maps incorporate $B_{1}$ inhomogeneity mapping to correct the $T_{1}$ maps before being delivered by the scanner.

\section{uVFA Results}

As expected, the uVFA maps performed the worst in terms of $T_{1}$ accuracy and uniformity as can be seen in Figure 4.1. The results of phantom experiments show, for a range of $T_{1}$ 's and experiment repetitions, that the uVFA maps differ from the reference TSE-IR $T_{1}$ maps by several hundred milliseconds. This represents an extremely 


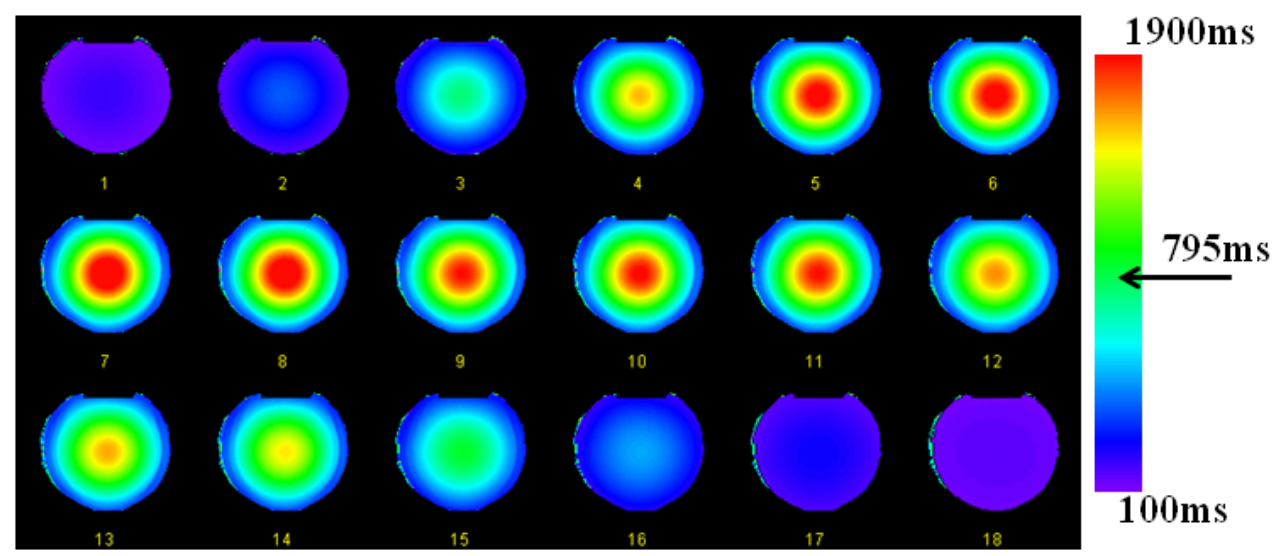

Figure 4.1: All 18 slices of a typical uVFA $T_{1}$ map. Reference $T_{1}$ as measured by TSE-IR is denoted by the arrow on the colour bar.

high degree of non-uniformity within individual slices. Note also that the $T_{1}$ values obtained with the uVFA method and average over the ROI are, in all cases, too high relative to the TSE-IR values.

Interslice uniformity is also highly variable. At first glance, one immediately notices the extreme underestimation of $T_{1}$ at the outer edges of the phantom (first and last few slices). This is common in many 3D sequences, such as 3D-FLASH, and is a result of "slab-profile" effects since the edges of the slab excited by the slab-selection process in the 3D-FLASH sequence are not sharply defined. These outer slices are normally ignored. This is being pointed out because the drop-off is not a fault of the VFA method itself, but rather the 3D-FLASH acquisition used to construct the VFA $T_{1}$ maps. However, even after ignoring these slices, there is still a substantial amount of interslice variation in the $\mathrm{uVFA} T_{1}$ maps.

It is clear from Table 4.1 that $\mathrm{uVFA} T_{1}$ mapping performs quite poorly. In terms of accuracy, the average percent error as calculated by averaging the results in the far right column of Table 4.1 was $24.6 \% \pm 11.3 \%$. Not only is the uVFA result in error by nearly $25 \%$, the uncertainly shows that the mean values measured by uVFA 


\begin{tabular}{lcccc}
\hline \multirow{2}{*}{ Experiment } & $\begin{array}{c}\text { Mean TSE-IR } \\
T_{1}(\mathrm{~ms})\end{array}$ & $\begin{array}{c}\text { Mean uVFA } \\
T_{1}(\mathrm{~ms})\end{array}$ & $\begin{array}{c}\text { Standard } \\
\text { Dev. }(\mathrm{ms})\end{array}$ & \% Error \\
\hline \multirow{2}{*}{ Oct 27, 2012 } & 1139 & 1387 & 434 & 21.8 \\
& 751 & 910 & 287 & 21.2 \\
& 527 & 633 & 244 & 20.1 \\
\hline Nov 10, 2012 & 1125 & 1371 & 426 & 21.9 \\
& 657 & 832 & 266 & 26.6 \\
\hline Mar 31, 2013 & 511 & 663 & 222 & 29.7 \\
& 1258 & 1885 & 722 & 49.8 \\
& 795 & 1101 & 410 & 38.5 \\
& 550 & 713 & 222 & 13.9 \\
\hline Jun 9, 2013 & 1265 & 1441 & 412 & 9.9 \\
& 634 & 697 & 205 & 12.3 \\
\hline
\end{tabular}

Table 4.1: Table showing results of the uVFA $T_{1}$ mapping method with reference to TSE-IR for three concentrations of contrast agent. Mean and standard deviation values were calculated by placing an ROI on the central slice of the phantom. 


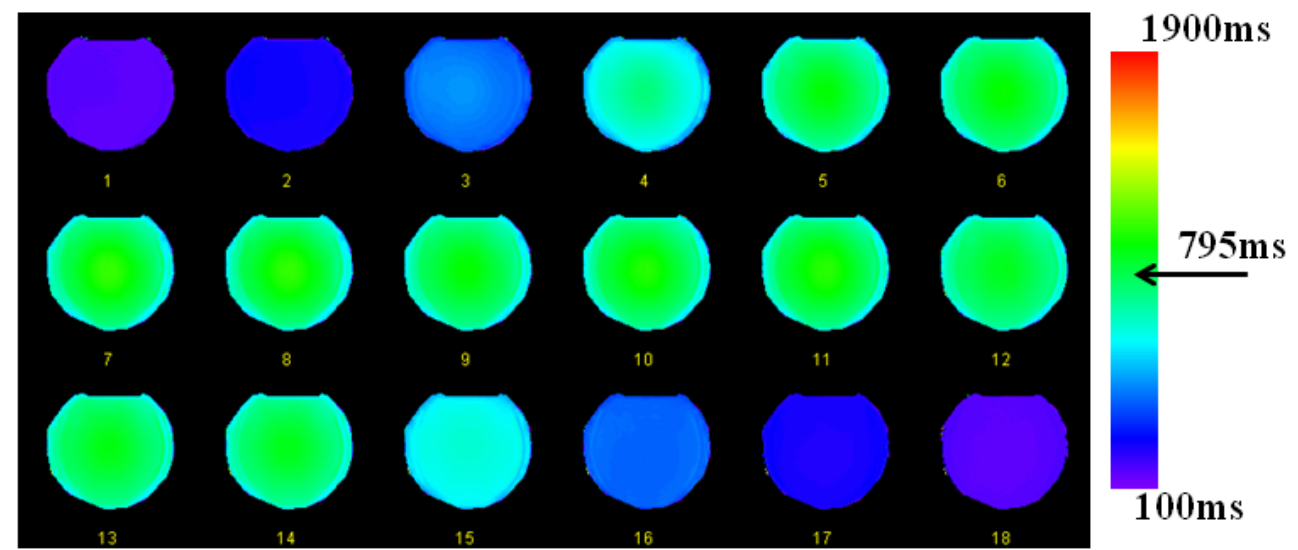

Figure 4.2: All 18 slices of a typical cVFA $T_{1}$ map. Reference $T_{1}$ as measured by TSE-IR is denoted by arrow on colourbar.

tend to vary wildy with $T_{1}$ and repeated measurement. This illustrates the unreliable nature of uncorrected VFA $T_{1}$ maps at $3 \mathrm{~T}$. In addition, the maps are extremely nonuniform with an average standard deviation within the ROI of $338 \mathrm{~ms} \pm 153 \mathrm{~ms}$. This number also fluctuates greatly between experiments, underlying the unpredictability and unreliability of these $T_{1}$ maps. As previously described in Section 3.3.2, this is, in part, due to the presence of a large amount of $B_{1}$ inhomogeneity on the $3 \mathrm{~T}$ MRI scanner used.

\section{cVFA Results}

The following results were obtained with cVFA $T_{1}$ mapping. By correcting for $B_{1}$ inhomogeneity, in-slice uniformity is increased greatly. This is visibly apparent in the images shown in Figure 4.2. The increased uniformity seen in the images brings the overall mean $T_{1}$ closer to the TSE-IR reference $T_{1}$. The results given in Table 4.2 indicate that $T_{1}$ values obtained with cVFA are again too high in all cases and the average percent error is calculated to be $7.95 \% \pm 7.23 \%$. This is a massive improvement over the uncorrected case, although the uncertainty remains quite high. In-slice 


\begin{tabular}{|c|c|c|c|c|}
\hline Experiment & $\begin{array}{c}\text { Mean TSE-IR } \\
T_{1}(\mathrm{~ms})\end{array}$ & $\begin{array}{c}\text { Mean cVFA } \\
T_{1}(\mathrm{~ms})\end{array}$ & $\begin{array}{c}\text { Standard } \\
\text { Dev. (ms) }\end{array}$ & $\%$ Error \\
\hline \multirow{3}{*}{ Oct 27,2012} & 1139 & 1162 & 96 & 2.02 \\
\hline & 751 & 807 & 40 & 7.46 \\
\hline & 527 & 566 & 29 & 7.40 \\
\hline \multirow{3}{*}{ Nov 10, 2012} & 1125 & 1162 & 77 & 3.29 \\
\hline & 657 & 703 & 65 & 7.00 \\
\hline & 511 & 547 & 68 & 7.04 \\
\hline \multirow{3}{*}{ Mar 31, 2013} & 1258 & 1554 & 157 & 23.5 \\
\hline & 795 & 946 & 78 & 19.0 \\
\hline & 550 & 622 & 45 & 13.1 \\
\hline \multirow{3}{*}{ Jun 9, 2013} & 1265 & 1204 & 118 & 4.82 \\
\hline & 634 & 639 & 54 & 0.789 \\
\hline & 469 & 469 & 49 & 0 \\
\hline
\end{tabular}

Table 4.2: Table showing results of the cVFA $T_{1}$ mapping method with reference to TSE-IR for three concentrations of contrast agent. Mean and standard deviation values were calculated by placing an ROI on the central slice of the phantom. 

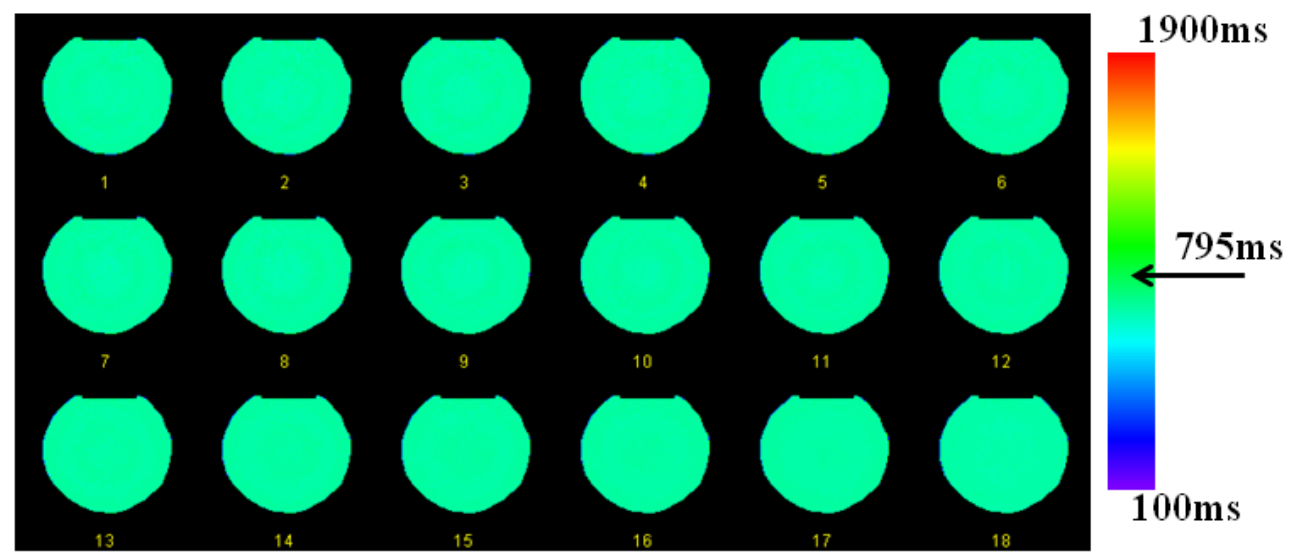

Figure 4.3: All 18 slices of a typical MOLLI $T_{1}$ map. Reference $T_{1}$ as measured by TSE-IR is denoted by the arrow on the colour bar.

uniformity is also significantly better. The average standard deviation within the ROI is reduced to $72 \pm 36 \mathrm{~ms}$. It is apparent from Table 4.2 that there is still a high degree of variability in the percent error as $T_{1}$ changes and between individual repetitions of the experiment. The percent errors range from a low of $0 \%$ to a high of $23.5 \%$, giving rise to the uncertainty seen above. Thus, overall reliability of the technique is still rather poor, with some results agreeing extremely well with reference values and others not very well.

\subsubsection{MOLLI Results}

In addition to VFA, a newly acquired MOLLI $T_{1}$ mapping sequence was reviewed for potential use in clinical DCE-MRI experiments. As was hoped for, MOLLI provided the most accurate $T_{1}$ mapping while maintaining a clinically practical acquisition time. The MOLLI sequence gives improved results in all areas of importance as seen in Figure 4.3. The images show a noticeably high level of homogeneity both in-slice and between slices. A high level of agreement with TSE-IR is also achieved. The results shown in Table 4.3 indicate an excellent improvement in $T_{1}$ mapping over 


\begin{tabular}{ccccc}
\hline \multirow{2}{*}{ Experiment } & $\begin{array}{c}\text { Mean TSE-IR } \\
T_{1}(\mathrm{~ms})\end{array}$ & $\begin{array}{c}\text { Mean MOLLI } \\
T_{1}(\mathrm{~ms})\end{array}$ & $\begin{array}{c}\text { Standard } \\
\text { Dev. }(\mathrm{ms})\end{array}$ & \% Error \\
\hline \multirow{2}{*}{ Mar 31, 2013 } & 1258 & 1257 & 15 & -0.08 \\
& 795 & 805 & 10 & 1.26 \\
& 550 & 562 & 8 & 2.18 \\
\hline Jun 9, 2013 & 1265 & 1256 & 18 & -0.71 \\
& 634 & 647 & 7 & 2.05 \\
& 496 & 509 & 6 & 2.62 \\
\hline Jun 15, 2013 & 1395 & 1393 & 10 & -0.14 \\
& 853 & 867 & 7 & 1.64 \\
& 574 & 585 & 5 & -0.17 \\
\hline Jun 29, 2013 & 1189 & 1187 & 10 & 1.55 \\
& 773 & 785 & 7 & 2.76 \\
\hline
\end{tabular}

Table 4.3: Table showing results of MOLLI $T_{1}$ mapping with reference to TSE-IR for three concentrations of contrast agent. Mean and standard deviation values were calculated by placing an ROI on the central slice of the phantom. 
both VFA methods. The average percent error is $1.24 \% \pm 1.20 \%$. This is a major improvement in overall accuracy and stability with changes in $T_{1}$ and with repeated measurements. In addition, the average standard deviation found within the ROI for the central slice is $9 \mathrm{~ms} \pm 4 \mathrm{~ms}$, yielding a high level of in-slice uniformity. In Table 4.3, the percent errors are extremly consistent and fluctuate by less than $4 \%$ altogether, indicating very high stability.

Note, that for each of the $T_{1}$ mapping methods, three values of $T_{1}$ were listed and compared to reference values in Tables 4.1, 4.2, and 4.3. The highest and lowest values represent the values of $T_{1 \text { pre }}$ and $T_{1 \text { post }}$, respectively. These are the values of $T_{1}$ that are directly inputted in to either the $T_{1 \text { pre }}$ or Bookend method to generate $[G d]$ maps. A third, intermediate value of $T_{1}$ was also included for the purposes of providing a comparison to reference somewhere between $T_{1 \text { pre }}$ and $T_{1 \text { post }}$. Figure 4.4 shows many intermediate values of $T_{1}$ for the three competing methods over a wide range of $T_{1}$. This shows that the results presented in this chapter accurately represent the trends in $T_{1}$ accuracy when more points in the $T_{1}$ range are sampled.

\subsection{Results of $[G d]$ Quantification}

As explained in Sections 2.2.2 and 2.2.3, $[G d]$ evaluation as a function of time is of great importance in DCE-MRI. In these phantom experiments, an ideal concentration map is one that is relatively homogeneous, both in-slice and between slices, with pixel values closely matching those of the reference concentration map. Recall from Sections 3.4.2 and 4.1 that reference $[G d]$ values were obtained using TSE-IR $T_{1}$ maps to calculate $[G d]$ through the direct use of Eq. (2.22). As outlined in Section 3.4.2, the measurements reported below represent the mean \pm standard deviation of $[G d]$ values within an ROI placed on the central slice. 


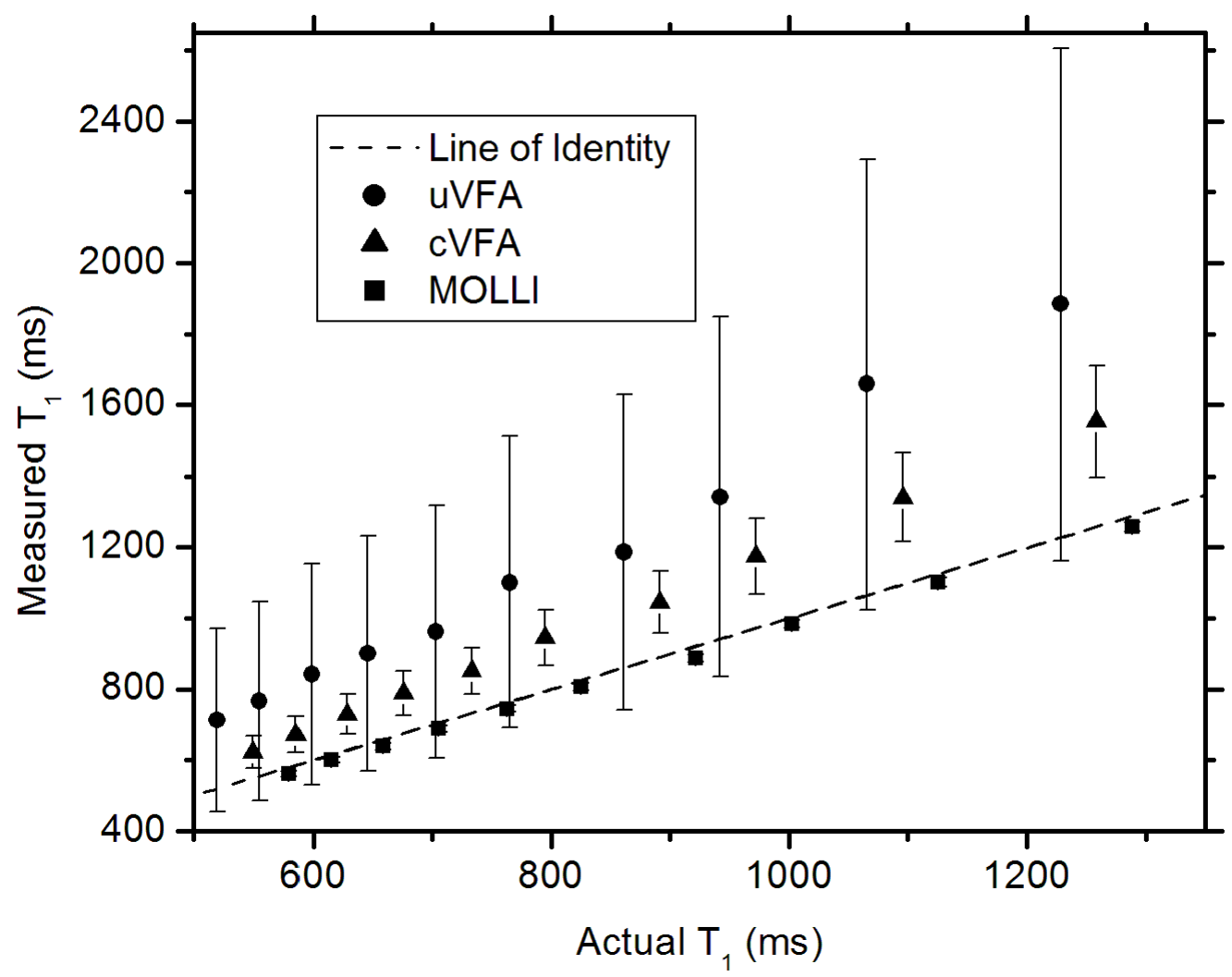

Figure 4.4: A comparison of all three $T_{1}$ mapping techniques over a large range of $T_{1}$ values. Points represent mean $T_{1}$ and error bars represent plus and minus one standard deviation within the ROI. 


\subsubsection{The $T_{1 p r e}$ Method}

The $[G d]$ quantification results to follow have been analyzed using the $T_{1 p r e}$ method as described in Section 2.3.2. As a reminder, the three $T_{1 p r e}$-based methods as outlined in Section 3.4.2 and Figure 3.8 are: $T_{1 p r e^{-}} \mathrm{uVFA}, T_{1 p r e^{-\mathrm{cVFA}}}$, and $T_{1 p r e}-\mathrm{LL}$, which use uVFA, cVFA, and MOLLI $T_{1}$ maps, respectively. The trendlines for each repetition of the experiment for a given method are plotted together on the same graph along with the TSE-IR reference concentrations. The slopes of the mean, minimum, and maximum trendlines are presented as described in Section 3.4.2.

\section{$T_{1 p r e}$-UVFA}

The results of the concentration analysis for the $T_{1 p r e}$-uVFA method are presented in Table 4.4. The resulting trends are shown in Figure 4.5. The slopes of the resulting trendlines are presented in Table 4.5.

The average percent error for the experiments ranged from $-36.5 \%$ to $-2.8 \%$. The results show significant spread in the trendlines of the $T_{1 p r e-u V F A}$ analysis. One experiment differs from reference by as much as $33 \%$ while another varies by as little as $4 \%$. As can be seen from the error bars for all trends in Figure 4.5 , the $T_{1 p r e-u V F A}$ method exhibits a high degree of non-uniformity within the images.

\section{$T_{1 p r e}$-cVFA}

The results of the concentration analysis for the $T_{1 p r e}$-cVFA method are presented in Table 4.6. The resulting trends are shown in Figure 4.6. The slopes of the resulting trendlines are presented in Table 4.7 .

The average percent error for the experiments ranged from $-21.6 \%$ to $23.2 \%$. The $T_{1 p r e^{-c V F A}}$ method shows improvement in over $T_{1 p r e} \mathrm{uVFA}$ some areas. In the case of in-plane variability, reflected by the error bars, this method is noticeably more 


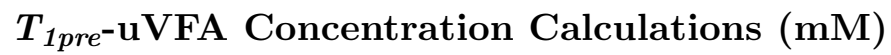

\begin{tabular}{|cc|cc|cc|cc|}
\hline \multicolumn{2}{c}{ Oct 27 } & \multicolumn{2}{c}{ Nov 10 } & \multicolumn{2}{c}{ Mar 31 } & \multicolumn{2}{c}{ Jun 9 } \\
\hline Actual & Measured & Actual & Measured & Actual & Measured & Actual & Measured \\
\hline 0.0 & $0.001 \pm 0.002$ & 0.0 & $0.001 \pm 0.001$ & 0.0 & $0.001 \pm 0.001$ & 0.0 & $0.001 \pm 0.002$ \\
0.030 & $0.026 \pm 0.009$ & 0.054 & $0.047 \pm 0.013$ & 0.030 & $0.011 \pm 0.004$ & 0.084 & $0.081 \pm 0.002$ \\
0.058 & $0.051 \pm 0.017$ & 0.110 & $0.092 \pm 0.024$ & 0.060 & $0.033 \pm 0.010$ & 0.140 & $0.143 \pm 0.039$ \\
0.086 & $0.064 \pm 0.019$ & 0.162 & $0.141 \pm 0.036$ & 0.082 & $0.060 \pm 0.018$ & 0.202 & $0.192 \pm 0.053$ \\
0.117 & $0.094 \pm 0.026$ & 0.221 & $0.193 \pm 0.048$ & 0.119 & $0.080 \pm 0.024$ & 0.261 & $0.250 \pm 0.069$ \\
0.142 & $0.131 \pm 0.035$ & 0.274 & $0.242 \pm 0.060$ & 0.146 & $0.102 \pm 0.030$ & 0.314 & $0.304 \pm 0.083$ \\
0.172 & $0.152 \pm 0.040$ & & & 0.176 & $0.115 \pm 0.034$ & & \\
0.198 & $0.166 \pm 0.043$ & & & 0.204 & $0.140 \pm 0.041$ & & \\
0.233 & $0.207 \pm 0.054$ & & & 0.235 & $0.159 \pm 0.046$ & & \\
0.262 & $0.230 \pm 0.058$ & & & 0.262 & $0.176 \pm 0.052$ & & \\
\hline
\end{tabular}

Table 4.4: Measured $[G d]$ results for the $T_{1 p r e}-\mathrm{uVFA}$ method. "Actual" columns represent reference values calculated using TSE-IR.

$T_{1 p r e^{-u V F A}}$

\begin{tabular}{ccccc}
\hline Slope of Trend & Oct 27 & Nov 10 & Mar 31 & Jun 9 \\
\hline Minimum & 0.64 & 0.66 & 0.48 & 0.70 \\
\hline Mean & 0.87 & 0.87 & 0.67 & 0.97 \\
\hline Maximum & 1.10 & 1.09 & 0.87 & 1.23 \\
\hline
\end{tabular}

Table 4.5: Slopes of the linear regression analysis of the mean, minimum, and maximum trends seen in Figure 4.5 


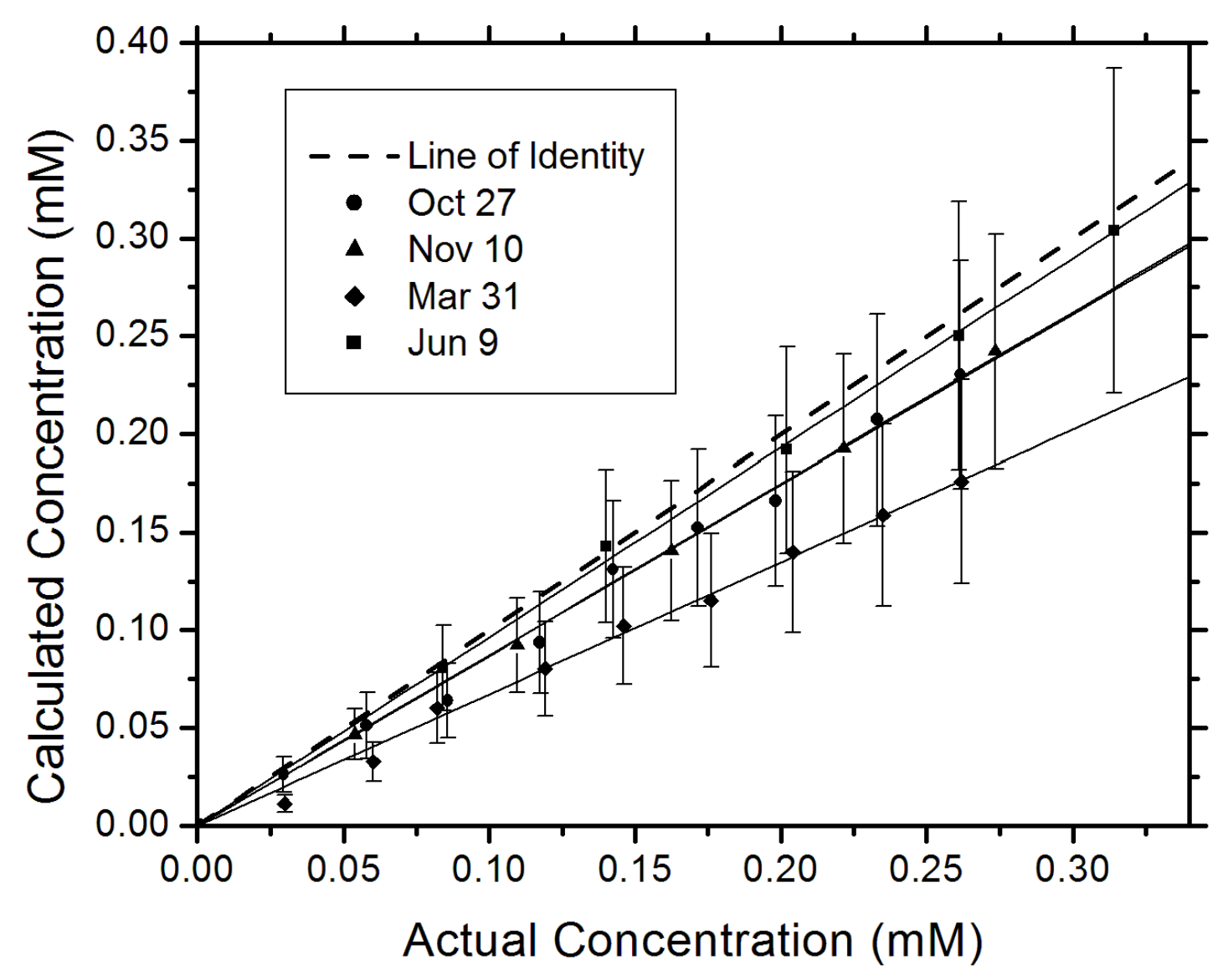

Figure 4.5: Trends of the $T_{1 p r e^{-u V F A}}$ analysis. 
$T_{1 p r e}$-cVFA Concentration Calculations $(\mathrm{mM})$

\begin{tabular}{|cc|cc|cc|cc|}
\hline \multicolumn{2}{c}{ Oct 27 } & \multicolumn{2}{c}{ Nov 10 } & \multicolumn{2}{c}{ Mar 31 } & \multicolumn{2}{c}{ Jun 9 } \\
\hline Actual & Measured & Actual & Measured & Actual & Measured & Actual & Measured \\
\hline 0.0 & $0.001 \pm 0.002$ & 0.0 & $0.001 \pm 0.002$ & 0.0 & $0.001 \pm 0.002$ & 0.0 & $0.001 \pm 0.002$ \\
0.030 & $0.034 \pm 0.008$ & 0.054 & $0.056 \pm 0.007$ & 0.030 & $0.014 \pm 0.004$ & 0.084 & $0.102 \pm 0.012$ \\
0.058 & $0.065 \pm 0.012$ & 0.110 & $0.112 \pm 0.012$ & 0.060 & $0.040 \pm 0.005$ & 0.140 & $0.181 \pm 0.020$ \\
0.086 & $0.081 \pm 0.013$ & 0.162 & $0.171 \pm 0.015$ & 0.082 & $0.074 \pm 0.007$ & 0.202 & $0.244 \pm 0.028$ \\
0.117 & $0.119 \pm 0.017$ & 0.221 & $0.235 \pm 0.020$ & 0.119 & $0.099 \pm 0.008$ & 0.261 & $0.319 \pm 0.036$ \\
0.142 & $0.166 \pm 0.022$ & 0.274 & $0.296 \pm 0.024$ & 0.146 & $0.127 \pm 0.010$ & 0.314 & $0.388 \pm 0.045$ \\
0.172 & $0.194 \pm 0.024$ & & & 0.176 & $0.143 \pm 0.012$ & & \\
0.198 & $0.212 \pm 0.026$ & & & 0.204 & $0.173 \pm 0.013$ & & \\
0.233 & $0.265 \pm 0.032$ & & & 0.235 & $0.197 \pm 0.013$ & & \\
0.262 & $0.295 \pm 0.034$ & & & 0.262 & $0.218 \pm 0.015$ & & \\
\hline
\end{tabular}

Table 4.6: Measured $[G d]$ results for the $T_{1 p r e} e^{-c V F A}$ method. "Actual" columns represent reference values calculated using TSE-IR.

$T_{1 p r e}{ }^{-c V F A}$

\begin{tabular}{ccccc}
\hline Slope of Trend & Oct 27 & Nov 10 & Mar 31 & Jun 9 \\
\hline Minimum & 0.97 & 0.97 & 0.77 & 1.08 \\
\hline Mean & 1.11 & 1.07 & 0.84 & 1.23 \\
\hline Maximum & 1.25 & 1.16 & 0.90 & 1.37 \\
\hline
\end{tabular}

Table 4.7: Slopes of the linear regression analysis of the mean, minimum, and maximum trends seen in Figure 4.6. 


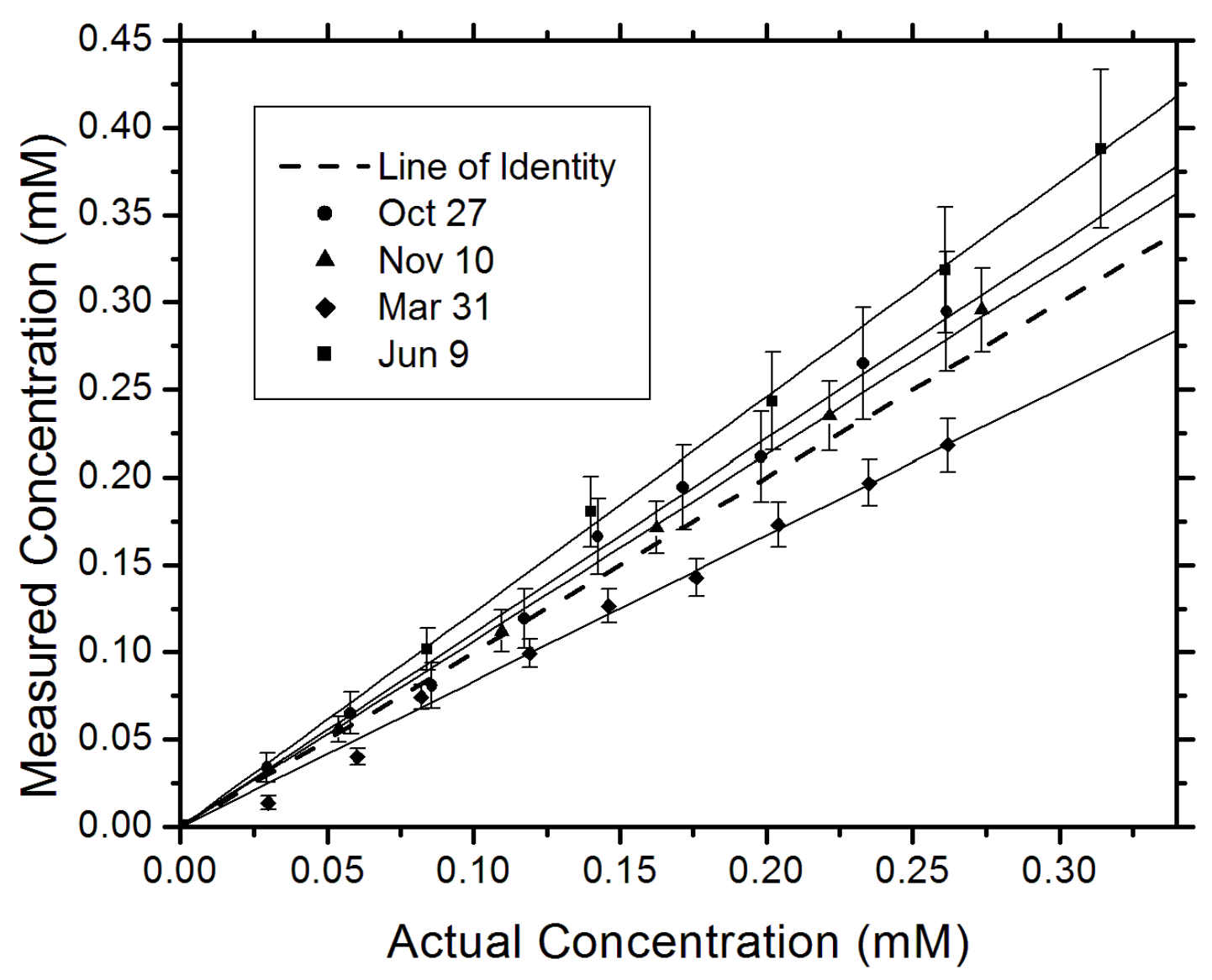

Figure 4.6: Trends of the $T_{1 p r e^{-c V F A}}$ analysis. 
$T_{1 p r e}$-LL Concentration Calculations (mM)

\begin{tabular}{|cc|cc|cc|cc|}
\hline \multicolumn{2}{c}{ Mar 31 } & \multicolumn{2}{c}{ Jun 9 } & \multicolumn{2}{c}{ Jun 15 } & \multicolumn{2}{c}{ Jun 29 } \\
\hline Actual & Measured & Actual & Measured & Actual & Measured & Actual & Measured \\
\hline 0.0 & $0.001 \pm 0.002$ & 0.0 & $0.001 \pm 0.002$ & 0.0 & $0.001 \pm 0.002$ & 0.0 & $0.001 \pm 0.002$ \\
0.030 & $0.019 \pm 0.005$ & 0.084 & $0.096 \pm 0.007$ & 0.029 & $0.016 \pm 0.006$ & 0.060 & $0.086 \pm 0.006$ \\
0.060 & $0.052 \pm 0.005$ & 0.140 & $0.170 \pm 0.012$ & 0.059 & $0.053 \pm 0.008$ & 0.117 & $0.151 \pm 0.009$ \\
0.082 & $0.096 \pm 0.006$ & 0.202 & $0.229 \pm 0.014$ & 0.088 & $0.084 \pm 0.010$ & 0.175 & $0.227 \pm 0.011$ \\
0.119 & $0.128 \pm 0.008$ & 0.261 & $0.300 \pm 0.018$ & 0.117 & $0.114 \pm 0.013$ & 0.235 & $0.298 \pm 0.014$ \\
0.146 & $0.164 \pm 0.008$ & 0.314 & $0.364 \pm 0.022$ & 0.148 & $0.148 \pm 0.015$ & 0.292 & $0.370 \pm 0.016$ \\
0.176 & $0.185 \pm 0.009$ & & & 0.176 & $0.176 \pm 0.018$ & & \\
0.204 & $0.225 \pm 0.010$ & & & 0.205 & $0.206 \pm 0.020$ & & \\
0.235 & $0.256 \pm 0.010$ & & & 0.234 & $0.234 \pm 0.023$ & & \\
0.262 & $0.284 \pm 0.013$ & & & 0.263 & $0.271 \pm 0.026$ & & \\
\hline
\end{tabular}

Table 4.8: Measured $[G d]$ results for the $T_{1 p r e}-\mathrm{LL}$ method. "Actual" columns represent reference values calculated using TSE-IR.

homogeneous than the uncorrected method. However, it is evident that the spread of the trendlines has not improved and trendline slopes vary from $23 \%$ overestimation to $16 \%$ underestimation with respect to reference concentration values.

\section{$T_{1 p r e}-\mathrm{LL}$}

The results of the concentration analysis for the $T_{1 p r e}$-LL method are presented in Table 4.8. The resulting trends are shown in Figure 4.7. The slopes of the resulting trendlines are presented in Table 4.9.

The average percent error for the experiments ranged from $-6.7 \%$ to $30.97 \%$. Once again, the $T_{1 p r e}$-LL method shows considerable improvement in overall inhomogeneity. But, as with the previous two $T_{1 p r e}$-based methods, the slopes of the trendlines tend to vary significantly. The trendlines exhibit a spread from $28 \%$ overestimation to $1 \%$ overestimation with respect to reference concentration values. 


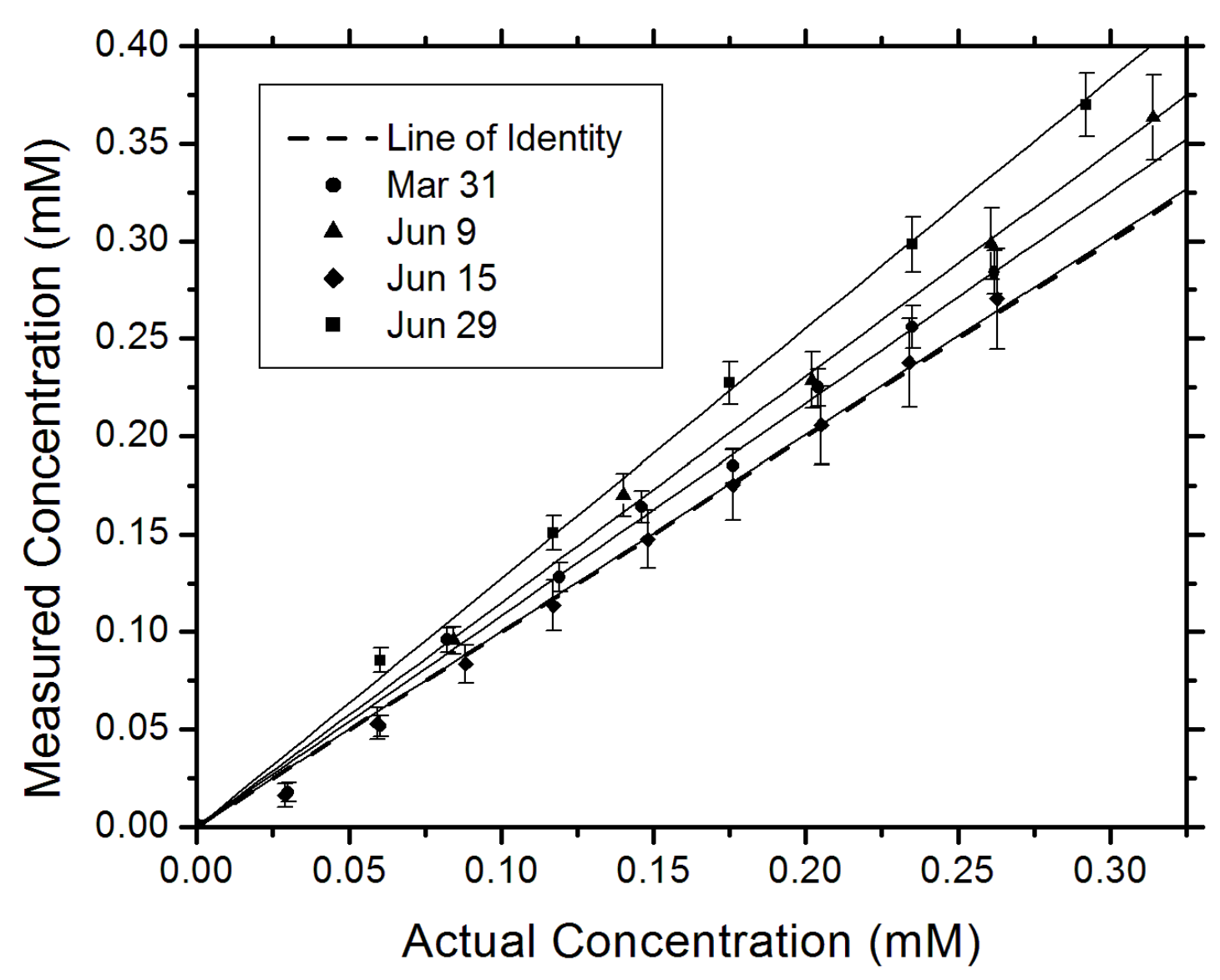

Figure 4.7: Trends of the $T_{1 p r e}-\mathrm{LL}$ analysis.

\begin{tabular}{ccccc}
$\boldsymbol{T}_{1 \text { pre }} \mathbf{- L L}$ & & & & \\
\hline Slope of Trend & Mar 31 & Jun 9 & Jun 15 & Jun 29 \\
\hline Minimum & 1.04 & 1.08 & 0.90 & 1.22 \\
\hline Mean & 1.08 & 1.15 & 1.01 & 1.28 \\
\hline Maximum & 1.13 & 1.22 & 1.11 & 1.34 \\
\hline
\end{tabular}

Table 4.9: Slopes of the linear regression analysis of the mean, minimum, and maximum trends seen in Figure 4.7. 
Overall, the $T_{1 \text { pre }}$-based methods did show some improvement as more accurate $T_{1}$ maps were used. However, the variability of the trends with repeated experiments is a cause for concern. Further analysis of the results presented here is performed in Chapter 5 .

\subsubsection{The Bookend Method}

The results to follow have been analyzed using the Bookend method as described in

Section 2.3.3. As a reminder, the three BE-based methods as outlined in Chapter 3.4.2 and Figure 3.8 are: BE-uVFA, BE-cVFA, and BE-LL, which use uVFA, cVFA, and MOLLI $T_{1}$ maps, respectively. The trendlines for each repetition of the experiment for a given method are plotted together on the same graph along with the TSE-IR reference concentrations. The slopes of the mean, minimum, and maximum trendlines are presented as described in Section 3.4.2.

\section{BE-uVFA}

The results of the concentration analysis for the BE-uVFA method are presented in Table 4.10. The resulting trends are shown in Figure 4.8. The slopes of the resulting trendlines are presented in Table 4.11. The average percent error for the experiments ranged from $-30.5 \%$ to $-11.8 \%$. The results for the BE-uVFA method are similar to the results of the $T_{1 p r e}-\mathrm{uVFA}$ method in terms of overall accuracy. The slopes of the resulting trendlines are seen to vary from $12 \%$ underestimation to $30 \%$ underestimation with respect to reference values. This is an improvement over the $T_{1 \text { pre }}$-uVFA method. However, the error bars in Figure 4.8 indicate that image uniformity is quite poor, mimicking its $T_{1 p r e}$ counterpart. 
BE-uVFA Concentration Calculations (mM)

\begin{tabular}{|cc|cc|cc|cc|}
\hline \multicolumn{2}{c}{ Oct 27 } & \multicolumn{2}{c}{ Nov 10 } & \multicolumn{2}{c}{ Mar 31 } & \multicolumn{2}{c}{ Jun 9 } \\
\hline Actual & Measured & Actual & Measured & Actual & Measured & Actual & Measured \\
\hline 0.0 & $0.001 \pm 0.001$ & 0.0 & $0.001 \pm 0.002$ & 0.0 & $0.001 \pm 0.002$ & 0.0 & $0.001 \pm 0.001$ \\
0.030 & $0.024 \pm 0.008$ & 0.054 & $0.037 \pm 0.011$ & 0.030 & $0.013 \pm 0.005$ & 0.084 & $0.074 \pm 0.022$ \\
0.058 & $0.046 \pm 0.015$ & 0.110 & $0.074 \pm 0.021$ & 0.060 & $0.039 \pm 0.011$ & 0.140 & $0.130 \pm 0.039$ \\
0.086 & $0.057 \pm 0.017$ & 0.162 & $0.113 \pm 0.032$ & 0.082 & $0.072 \pm 0.020$ & 0.202 & $0.174 \pm 0.051$ \\
0.117 & $0.084 \pm 0.023$ & 0.221 & $0.155 \pm 0.043$ & 0.119 & $0.096 \pm 0.026$ & 0.261 & $0.227 \pm 0.067$ \\
0.142 & $0.118 \pm 0.032$ & 0.274 & $0.194 \pm 0.053$ & 0.146 & $0.123 \pm 0.033$ & 0.314 & $0.276 \pm 0.081$ \\
0.172 & $0.138 \pm 0.037$ & & & 0.176 & $0.139 \pm 0.038$ & & \\
0.198 & $0.150 \pm 0.040$ & & & 0.204 & $0.169 \pm 0.046$ & & \\
0.233 & $0.188 \pm 0.050$ & & & 0.235 & $0.192 \pm 0.051$ & & \\
0.262 & $0.209 \pm 0.055$ & & & 0.262 & $0.213 \pm 0.058$ & & \\
\hline
\end{tabular}

Table 4.10: Measured $[G d]$ results for the BE-uVFA method. "Actual" columns represent reference values calculated using TSE-IR.

\section{BE-uVFA}

\begin{tabular}{ccccc}
\hline Slope of Trend & Oct 27 & Nov 10 & Mar 31 & Jun 9 \\
\hline Minimum & 0.57 & 0.51 & 0.59 & 0.62 \\
\hline Mean & 0.79 & 0.70 & 0.81 & 0.88 \\
\hline Maximum & 1.00 & 0.90 & 1.03 & 1.13 \\
\hline
\end{tabular}

Table 4.11: Slopes of the linear regression analysis of the mean, minimum, and maximum trends seen in Figure 4.8. 


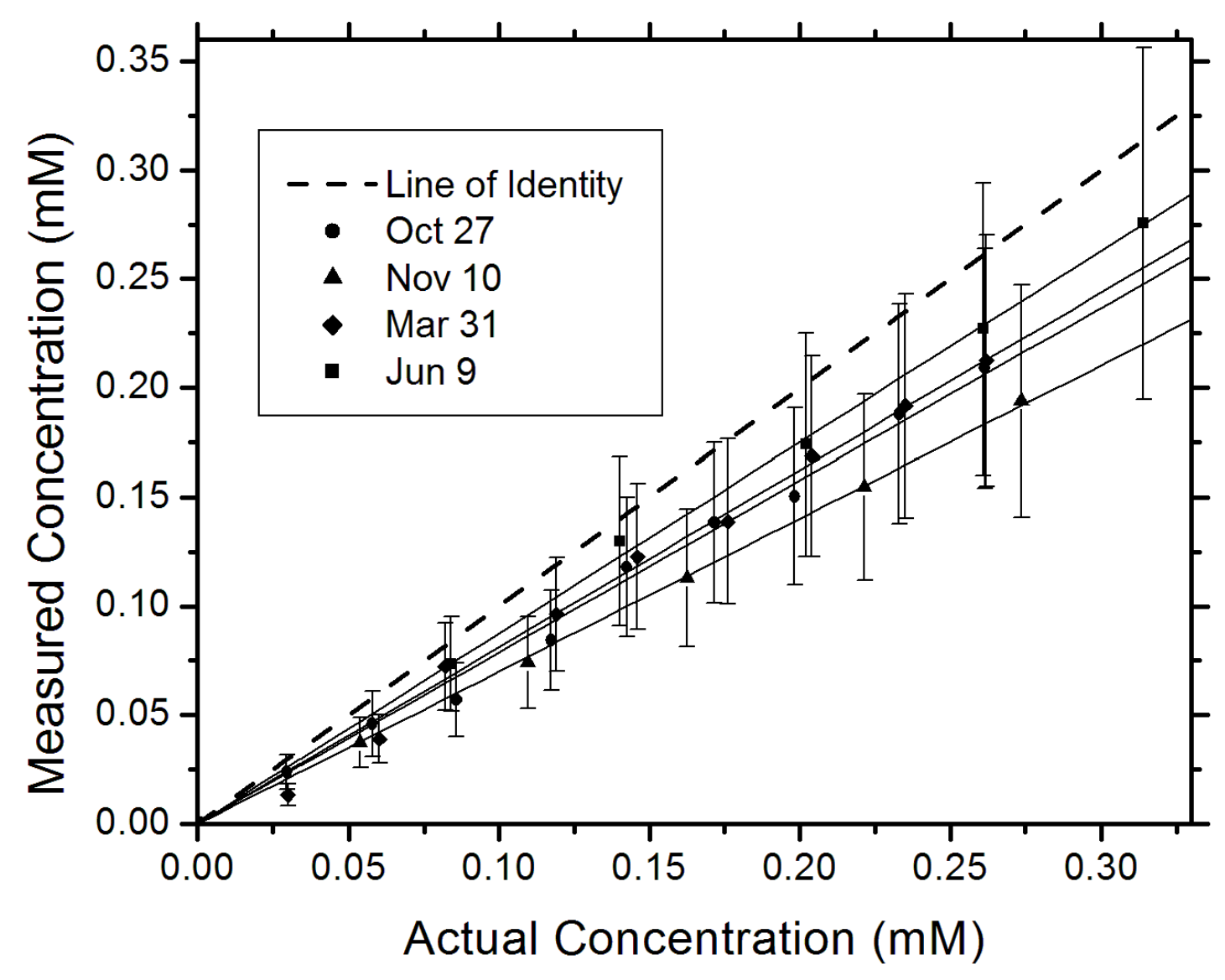

Figure 4.8: Trends of the BE-uVFA analysis. 
BE-cVFA Concentration Calculations (mM)

\begin{tabular}{|cc|cc|cc|cc|}
\hline \multicolumn{2}{c}{ Oct 27 } & \multicolumn{2}{c}{ Nov 10 } & \multicolumn{2}{c}{ Mar 31 } & \multicolumn{2}{c}{ Jun 9 } \\
\hline Actual & Measured & Actual & Measured & Actual & Measured & Actual & Measured \\
\hline 0.0 & $0.001 \pm 0.001$ & 0.0 & $0.001 \pm 0.001$ & 0.0 & $0.001 \pm 0.002$ & 0.0 & $0.001 \pm 0.002$ \\
0.030 & $0.026 \pm 0.006$ & 0.054 & $0.048 \pm 0.006$ & 0.030 & $0.015 \pm 0.004$ & 0.084 & $0.080 \pm 0.005$ \\
0.058 & $0.050 \pm 0.008$ & 0.110 & $0.095 \pm 0.007$ & 0.060 & $0.044 \pm 0.004$ & 0.140 & $0.141 \pm 0.009$ \\
0.086 & $0.062 \pm 0.009$ & 0.162 & $0.145 \pm 0.009$ & 0.082 & $0.082 \pm 0.006$ & 0.202 & $0.189 \pm 0.011$ \\
0.117 & $0.092 \pm 0.011$ & 0.221 & $0.200 \pm 0.010$ & 0.119 & $0.109 \pm 0.007$ & 0.261 & $0.247 \pm 0.013$ \\
0.142 & $0.128 \pm 0.012$ & 0.274 & $0.250 \pm 0.013$ & 0.146 & $0.140 \pm 0.007$ & 0.314 & $0.300 \pm 0.017$ \\
0.172 & $0.150 \pm 0.013$ & & & 0.176 & $0.158 \pm 0.008$ & & \\
0.198 & $0.163 \pm 0.014$ & & & 0.204 & $0.192 \pm 0.010$ & & \\
0.233 & $0.204 \pm 0.016$ & & & 0.235 & $0.218 \pm 0.010$ & & \\
0.262 & $0.227 \pm 0.018$ & & & 0.262 & $0.242 \pm 0.011$ & & \\
\hline
\end{tabular}

Table 4.12: Measured $[G d]$ results for the BE-cVFA method. "Actual" columns represent reference values calculated using TSE-IR.

\section{BE-cVFA}

The results of the concentration analysis for the BE-uVFA method are presented in Table 4.12. The resulting trends are shown in Figure 4.9. The slopes of the resulting trendlines are presented in Table 4.13.

The average percent error for the experiments ranged from $-15.7 \%$ to $-4.1 \%$. The BE-cVFA case shows considerable improvement, both over the BE-uVFA method and its counterpart, the $T_{1 p r e}$-cVFA method. The image uniformity is noticeably better, reflected by the decreased error bar size in Figure 4.9. Also, the trendline variations with repetition of the experiment are far more stable than any method shown up to this point. The trendlines vary from a low of $5 \%$ underestimation to a high of $14 \%$ underestimation with respect to reference values. 


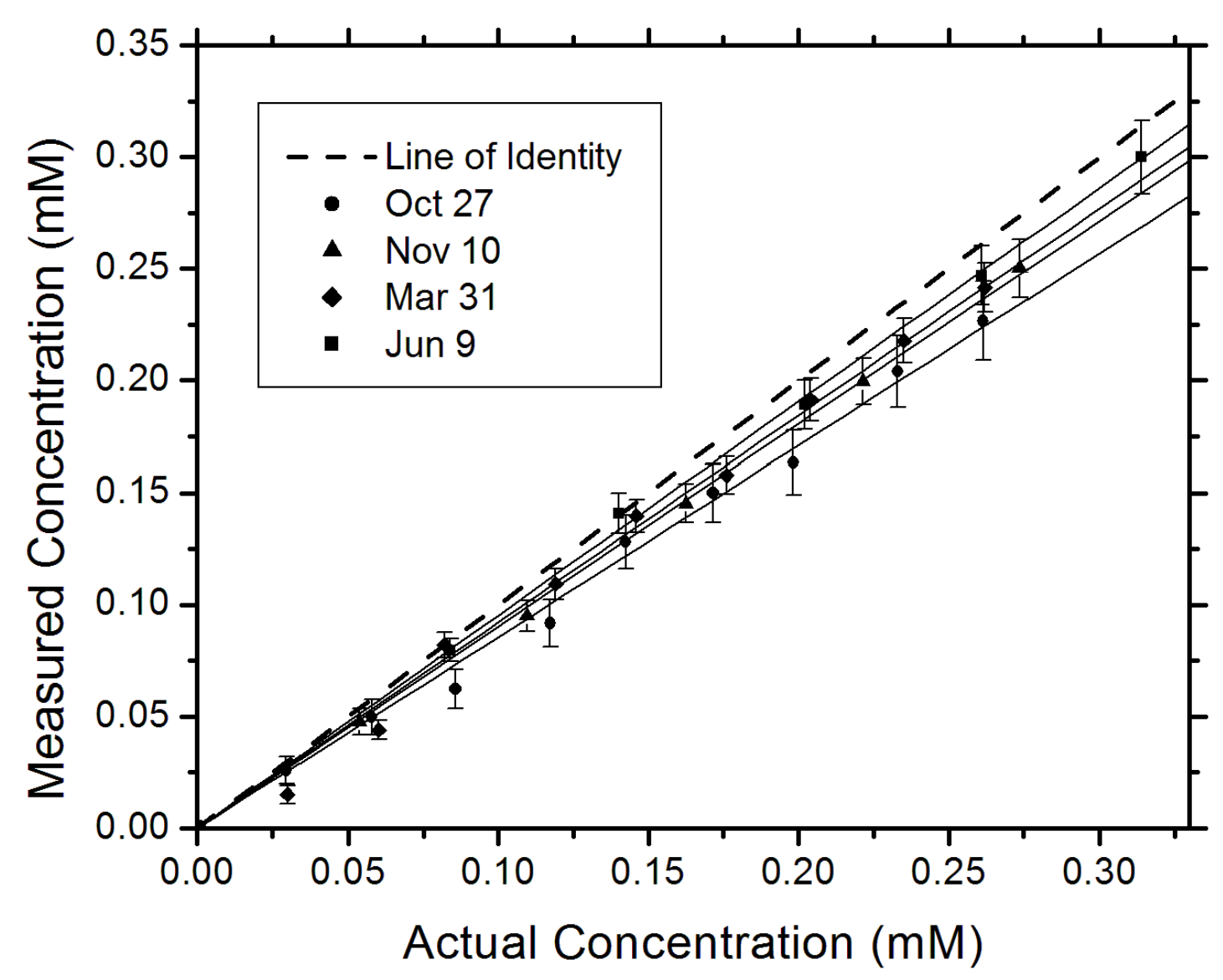

Figure 4.9: Trends of the BE-cVFA analysis.

BE-cVFA

\begin{tabular}{ccccc}
\hline Slope of Trend & Oct 27 & Nov 10 & Mar 31 & Jun 9 \\
\hline Minimum & 0.78 & 0.85 & 0.88 & 0.90 \\
\hline Mean & 0.86 & 0.90 & 0.92 & 0.95 \\
\hline Maximum & 0.93 & 0.95 & 0.97 & 1.00 \\
\hline
\end{tabular}

Table 4.13: Slopes of the linear regression analysis of the mean, minimum, and maximum trends seen in Figure 4.9. 
BE-LL Concentration Calculations ( $\mathrm{mM})$

\begin{tabular}{|cc|cc|cc|cc|}
\hline \multicolumn{2}{c}{ Mar 31 } & \multicolumn{2}{c}{ Jun 9 } & \multicolumn{2}{c}{ Jun 15 } & \multicolumn{2}{c}{ Jun 29 } \\
\hline Actual & Measured & Actual & Measured & Actual & Measured & Actual & Measured \\
\hline 0.0 & $0.001 \pm 0.002$ & 0.0 & $0.001 \pm 0.002$ & 0.0 & $0.001 \pm 0.002$ & 0.0 & $0.001 \pm 0.001$ \\
0.030 & $0.016 \pm 0.004$ & 0.084 & $0.080 \pm 0.003$ & 0.029 & $0.015 \pm 0.005$ & 0.060 & $0.065 \pm 0.004$ \\
0.060 & $0.046 \pm 0.004$ & 0.140 & $0.141 \pm 0.004$ & 0.059 & $0.049 \pm 0.004$ & 0.117 & $0.114 \pm 0.004$ \\
0.082 & $0.085 \pm 0.004$ & 0.202 & $0.189 \pm 0.004$ & 0.088 & $0.078 \pm 0.004$ & 0.175 & $0.171 \pm 0.004$ \\
0.119 & $0.114 \pm 0.005$ & 0.261 & $0.245 \pm 0.005$ & 0.117 & $0.106 \pm 0.005$ & 0.235 & $0.224 \pm 0.005$ \\
0.146 & $0.145 \pm 0.005$ & 0.314 & $0.300 \pm 0.005$ & 0.148 & $0.138 \pm 0.005$ & 0.292 & $0.277 \pm 0.005$ \\
0.176 & $0.164 \pm 0.005$ & & & 0.176 & $0.163 \pm 0.004$ & & \\
0.204 & $0.199 \pm 0.006$ & & & 0.205 & $0.191 \pm 0.004$ & & \\
0.235 & $0.227 \pm 0.006$ & & & 0.234 & $0.221 \pm 0.005$ & & \\
0.262 & $0.252 \pm 0.007$ & & & 0.263 & $0.252 \pm 0.005$ & & \\
\hline
\end{tabular}

Table 4.14: Measured $[G d]$ results for the BE-LL method. "Actual" columns represent reference values calculated using TSE-IR.

\section{BE-LL}

The results of the concentration analysis for the BE-LL method are presented in Table 4.14. The resulting trends are shown in Figure 4.10. The slopes of the resulting trendlines are presented in Table 4.15.

The average percent error for the experiments ranged from $-12.9 \%$ to $-1.5 \%$. The final method tested, BE-LL, provided excellent results in all areas of importance. As is evident in Figure 4.10, the trends are highly accurate with respect to the line of identity and repeated experiments provided very consistent results. The slopes ranged from a low of $4 \%$ underestimation to a high of $6 \%$ underestimation with repect to reference values. In addition, it is clear that inhomogeneity within the concentration maps is greatly reduce by using this method. The BE-LL concentration maps represent the most accurate, homogeneous, and repeatable maps calculated in 


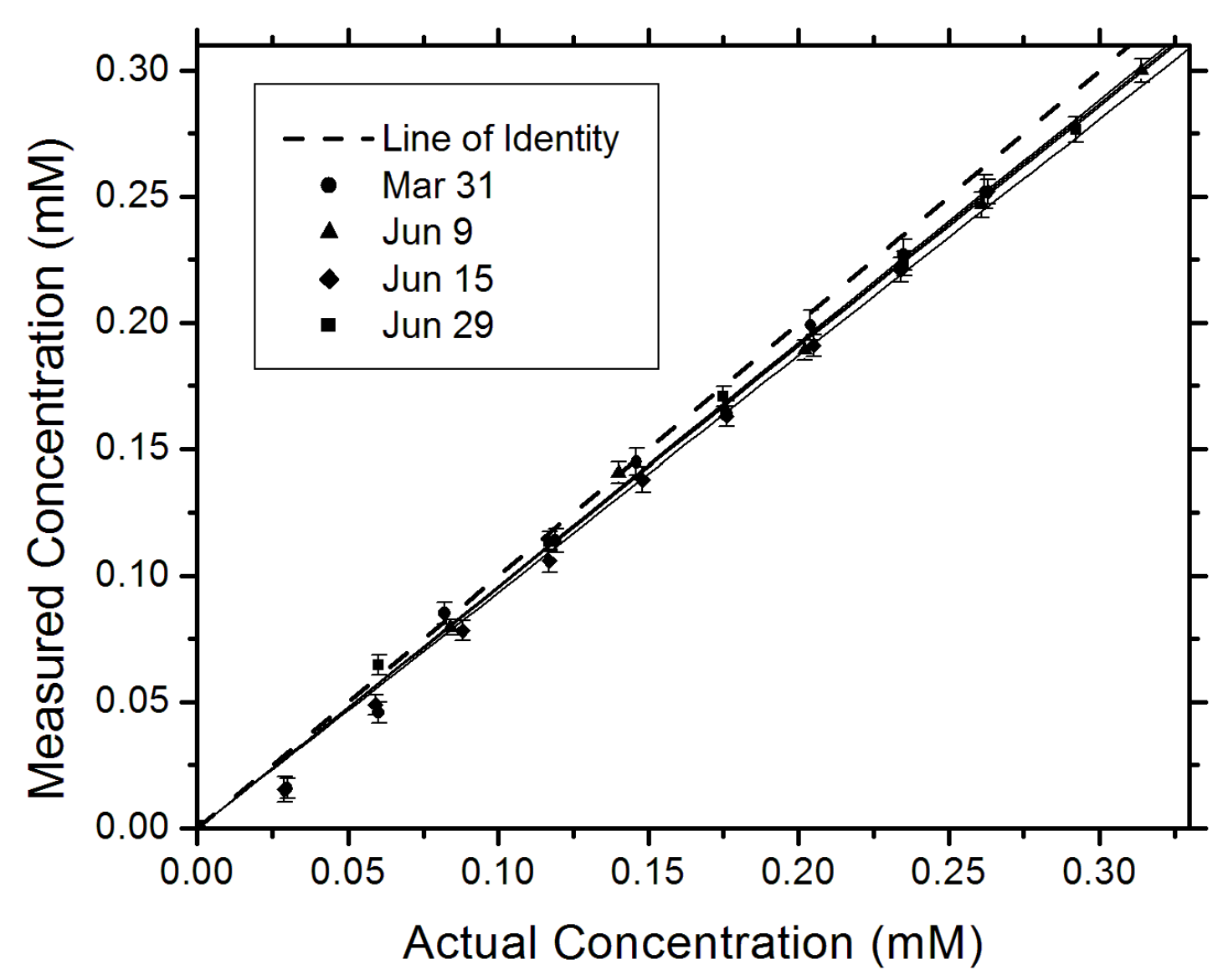

Figure 4.10: Trends of the BE-LL analysis.

BE-LL

\begin{tabular}{ccccc}
\hline Slope of Trend & Mar 31 & Jun 9 & Jun15 & Jun 29 \\
\hline Minimum & 0.93 & 0.93 & 0.91 & 0.94 \\
\hline Mean & 0.96 & 0.95 & 0.94 & 0.96 \\
\hline Maximum & 0.99 & 0.97 & 0.96 & 0.98 \\
\hline
\end{tabular}

Table 4.15: Slopes of the linear regression analysis of the mean, minimum, and maximum trends seen in Figure 4.10. 
$T_{1 p r e}$ Interslice $[G d]$ Comparison

\begin{tabular}{cccc} 
Slice Number & $T_{1 p r e}$-uVFA & $T_{1 p r e}$-cVFA & $T_{1 p r e}$-LL \\
5 & $-17.4 \pm 29.4$ & $-15.7 \pm 9.4$ & $-15.2 \pm 5.5$ \\
6 & $-4.9 \pm 30.4$ & $-2.4 \pm 11.0$ & $-0.6 \pm 6.7$ \\
7 & $-4.9 \pm 29.4$ & $-3.9 \pm 12.6$ & $9.6 \pm 7.3$ \\
8 & $-8.8 \pm 30.4$ & $-7.9 \pm 11.8$ & $3.7 \pm 8.5$ \\
9 & $0.0 \pm 29.4$ & $0.0 \pm 7.1$ & $0.0 \pm 4.9$ \\
10 & $1.0 \pm 29.4$ & $-3.1 \pm 7.9$ & $4.9 \pm 5.5$ \\
11 & $-3.9 \pm 30.4$ & $-9.4 \pm 9.4$ & $1.8 \pm 4.9$ \\
12 & $2.0 \pm 32.4$ & $-5.5 \pm 9.4$ & $-7.9 \pm 6.1$ \\
13 & $8.8 \pm 31.4$ & $-3.1 \pm 7.9$ & $-4.9 \pm 5.5$ \\
14 & $22.5 \pm 31.4$ & $3.9 \pm 7.9$ & $7.9 \pm 7.3$ \\
\hline
\end{tabular}

Table 4.16: Percent error plus and minus the standard deviation within ROI for slices 5-14 for the $T_{1 p r e}$ method relative to slice 9 .

this experiment.

As is evident in Figures 4.8, 4.9, and 4.10, the calculation of $[G d]$ values with the Bookend method becomes increasingly more accurate, uniform, and reproducible as $T_{1}$ accuracy is improved. While none of the $T_{1 p r e}$-based methods gave acceptable results, values obtained with the BE-LL method are all very good in terms of accuracy, uniformity, and reproducibility. Further analysis of these results is presented in Chapter 5 .

\subsubsection{Interslice Variations}

As discussed in Section 3.4.2, the interslice homogeneity of each $[G d]$ quantification method was also investigated. The results of this analysis for the $T_{1 p r e}$ method may be found in Table 4.16 and Figure 4.11. In this figure only slices 5 to 14 are shown.

The outer edges of the phantom exhibit extreme inhomogeneity due to the slab- 


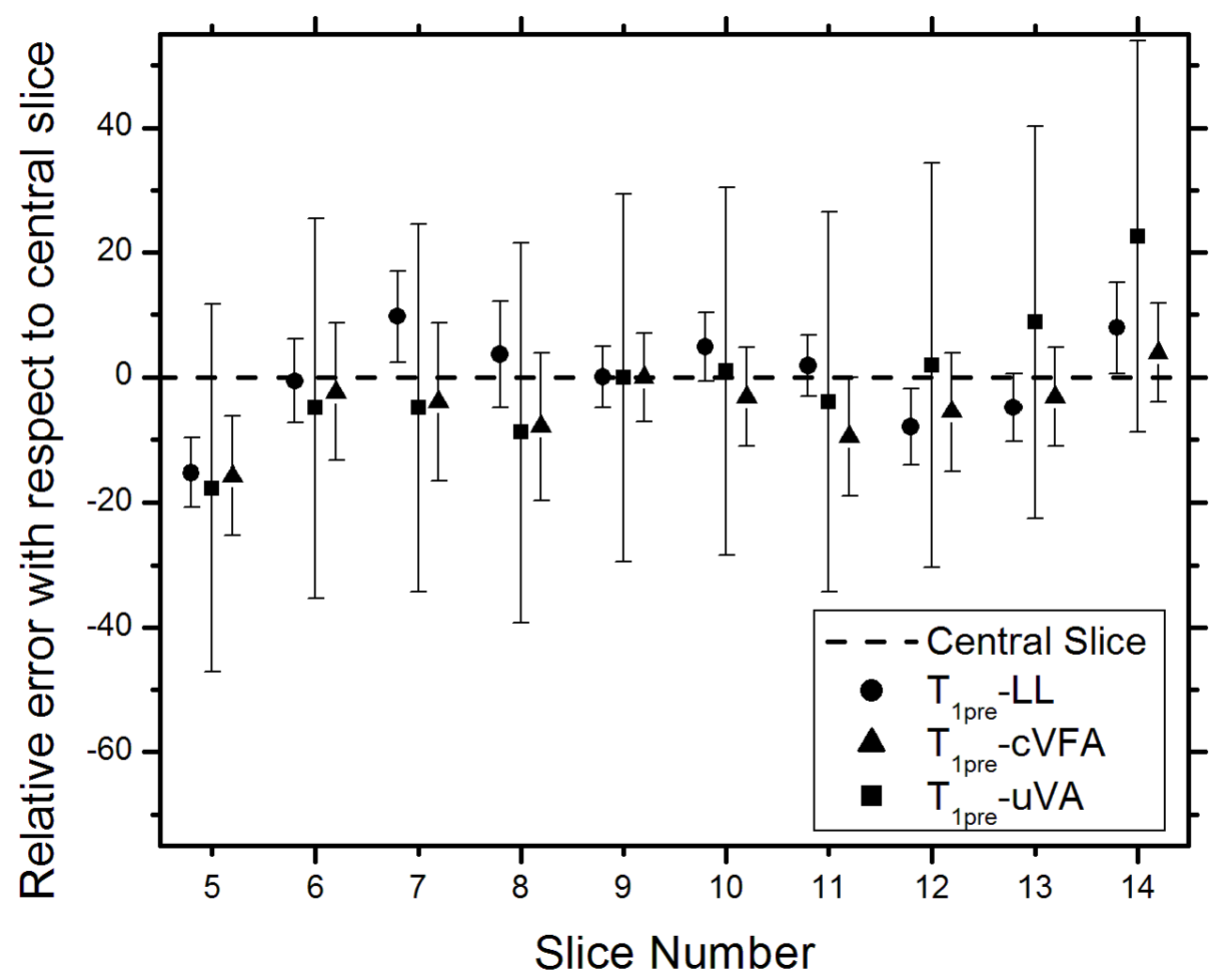

Figure 4.11: Interslice variation for the $T_{1 \text { pre }}$-based methods for slices 5-14. Points represent mean percent error with respect to central slice. Error bars represent plus and minus one standard deviation. 


\begin{tabular}{cccc}
\multicolumn{4}{c}{ Bookend interslice $[\boldsymbol{G d}]$ comparison } \\
\hline Slice Number & BE-uVFA & BE-cVFA & BE-LL \\
5 & $3.3 \pm 28.4$ & $6.5 \pm 5.8$ & $1.4 \pm 4.1$ \\
6 & $-0.81 \pm 26.8$ & $1.4 \pm 5.1$ & $2.1 \pm 3.4$ \\
7 & $1.6 \pm 27.6$ & $4.3 \pm 5.8$ & $0.7 \pm 3.4$ \\
8 & $1.6 \pm 27.6$ & $2.9 \pm 5.8$ & $-0.7 \pm 3.4$ \\
9 & $0.0 \pm 26.8$ & $0.0 \pm 5.1$ & $0.0 \pm 3.4$ \\
10 & $3.3 \pm 26.8$ & $2.2 \pm 5.8$ & $0.7 \pm 3.4$ \\
11 & $8.1 \pm 27.6$ & $4.3 \pm 5.8$ & $-2.1 \pm 3.4$ \\
12 & $8.9 \pm 27.6$ & $1.4 \pm 5.8$ & $-1.4 \pm 3.4$ \\
13 & $9.6 \pm 27.6$ & $0.7 \pm 5.8$ & $-1.4 \pm 3.4$ \\
14 & $23.6 \pm 30.1$ & $8.7 \pm 5.8$ & $-2.74 \pm 4.1$ \\
\hline
\end{tabular}

Table 4.17: Percent error plus and minus the standard deviation within the ROI for slices 5-14 for the BE method relative to slice 9 .

profile effect of 3D acquisitions described earlier and are typically ignored clinically. As can be seen, there is a considerable amount of variation from slice to slice for all three $T_{1 \text { pre }}$ based methods. It is observed that the interslice variations decrease with increasing $T_{1}$ accuracy. In addition, the standard deviations at each slice decrease rapidly with $T_{1}$ accuracy. There is no discernible trend in percent error and standard deviation as a function of slice number.

The interslice analysis for the Bookend method is found in Table 4.17 and Figure 4.12. The results for the Bookend method are noticeably more stable than the results for the $T_{1 \text { pre }}$ method. The results are very stable until approaching the edge of the phantom in slice 14 . Once again, no trend in percent error or standard deviation with slice number was discernible. 


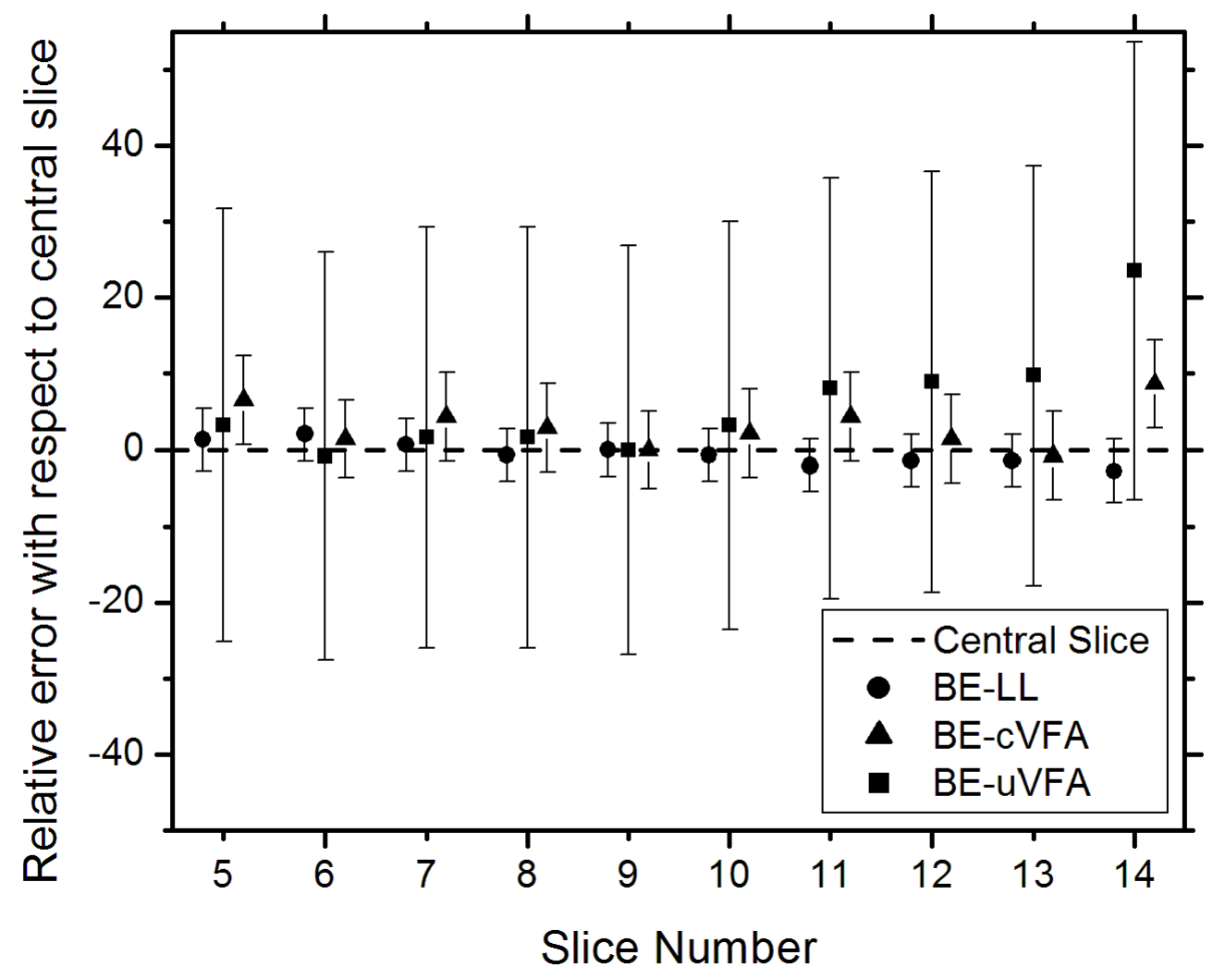

Figure 4.12: Interslice variation of the BE-based methods for slices 5-14. Points represent mean percent error with respect to central slice. Error bars represent plus and minus one standard deviation. 


\section{Chapter 5}

\section{Analysis and Discussion}

This chapter is dedicated to the analysis and interpretation of the results presented in Chapter 4. A brief analysis of the $T_{1}$ quantification will be performed in order to understand the trends in $T_{1}$ accuracy. The majority of the chapter will be dedicated to the discussion of the $[G d]$ quantification results. The behaviour of both the Bookend and $T_{1 \text { pre }}$ methods with $T_{1}$ accuracy will be analyzed and quantified.

\section{$5.1 \quad T_{1}$ Quantification and Accuracy}

The results presented in Section 4.2 indicate that the measurements obtained using the three difference $T_{1}$ mapping techniques used in this experiment differ greatly from one another. The uncorrected VFA method is clearly the worst of the three. Without the incorporation of $B_{1}$ corrections, the observed results tend to be inaccurate with respect to reference values, and tend to vary wildly between experiments. The corrected VFA method saw some improvement over its uncorrected counterpart, both in stability and overall accuracy. But, it is the MOLLI $T_{1}$ mapping technique that truly shines. This technique is shown to be both accurate and stable. Since both the $T_{1 \text { pre }}$ and Bookend methods for obtaining $[G d]$ rely so heavily on input parametric $T_{1}$ 


\begin{tabular}{cccc} 
& UVFA & cVFA & MOLLI \\
\hline Average \% Error & $24.6 \pm 11.3$ & $7.95 \pm 7.23$ & $1.24 \pm 1.20$ \\
\hline
\end{tabular}

Table 5.1: Average percent error with respect to TSE-IR reference $T_{1}$ values. The above are calculated from Tables 4.1, 4.2, and 4.3.

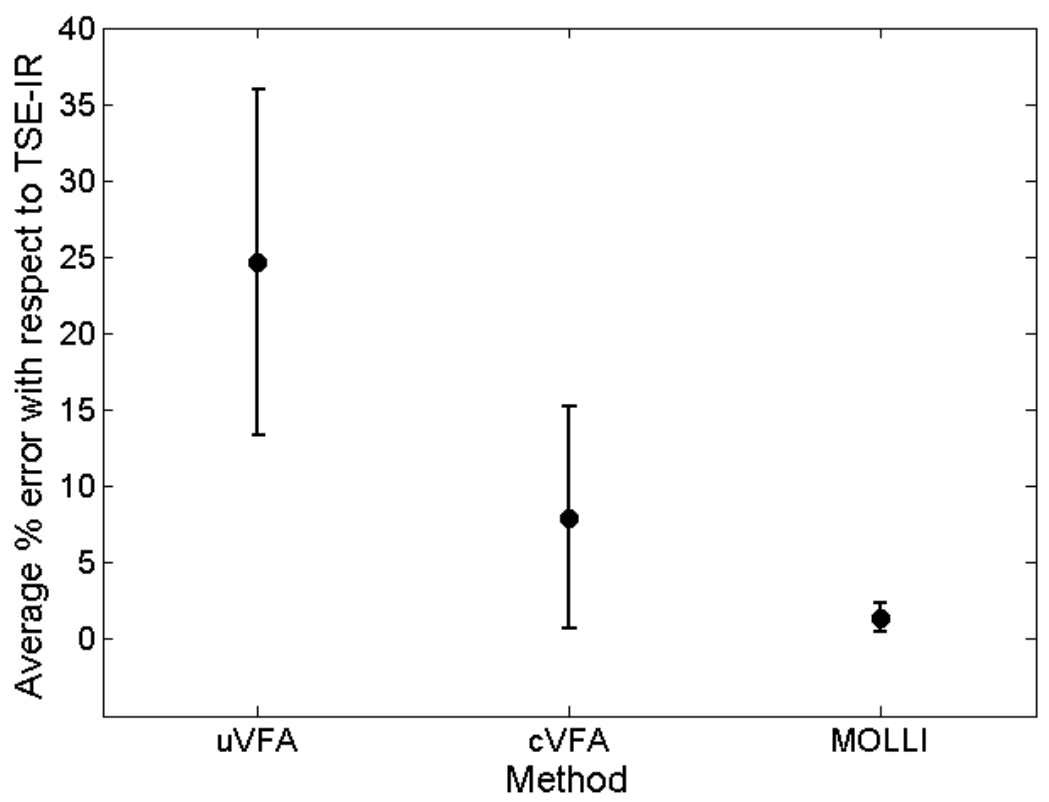

Figure 5.1: Average percent errors as a function of $T_{1}$ accuracy.

maps, it is expected that this $T_{1}$ mapping method will prove invaluable when converting raw 3D-FLASH signal to concentration. Table 5.1, where the average percent errors are given for the three methods considered, shows that there is a massive improvement in $T_{1}$ accuracy when moving from uVFA, to cVFA, and finally to MOLLI. The average percent error over several values of $T_{1}$ and over several repetitions drops dramatically. In addition, the variability of these averages, which is calculated as the standard deviation and reflected by the errors reported in Table 5.1, also drops and becomes extremely small. This is presented visually in Figure 5.1. From the uVFA case to the MOLLI case, the average percent error dropped dramatically (by over a full order of magnitude) from approximately $25 \%$ to $1 \%$. The variation in 


\section{UVFA CVFA MOLLI}

$\begin{array}{llll}\text { Average Standard Deviation(ms) } & 338 \pm 153 & 72 \pm 36 & 9 \pm 4\end{array}$

Table 5.2: Average standard deviations for the three $T_{1}$ mapping methods. The above are calculated from Tables 4.1, 4.2, and 4.3. The values reported are the mean standard deviation values plus and minus one standard deviation

these values also decreased by a significant margin, as is indicated by the error bars in Figure 5.1. The standard deviations of the percent errors decrease from a high of approximately $11 \%$ in the uVFA case, to a low of approximately $1 \%$. This represents another order of magnitude drop. By switching from the uVFA case to the MOLLI $T_{1}$ mapping method, a ten-fold reduction in percent error and variation with repetition is observed. This is in large part due to the fact that the uVFA case disregards any $B_{1}$ effects completely. However, it can be seen that there is still a rather large improvement when using MOLLI over the cVFA case. The cVFA method reduces the average percent error by a large amount, but the varaitions in this average with $T_{1}$ and repetition are similar in scale to the uncorrected case (around 8\% versus $11 \%$ respectively).

The above analysis determines the overall accuracy within the ROI of each of the three $T_{1}$ mapping techniques. Here the precision, determined from the standard deviation of of $T_{1}$ values within the ROI, will also be analyzed. The overall standard deviations of the $T_{1}$ results are calculated from the results in Section 4.2. The results presented in Table 5.2 indicate the average standard deviation of $T_{1}$ values. Echoing the previous results, a massive improvement in overall precision is observed when MOLLI is employed. The increase in precision is reflected by the drop in standard deviation values within the ROI from $338 \mathrm{~ms}$ to $9 \mathrm{~ms}$. Variations in average precision, reflected by the errors in Table 5.2 and error bars in Figure 5.2 are also observed to decrease. In terms of $T_{1}$ precision within the ROI, MOLLI is shown to provide extremely uniform $T_{1}$ maps that are very stable with both changes in $T_{1}$ and experiment 


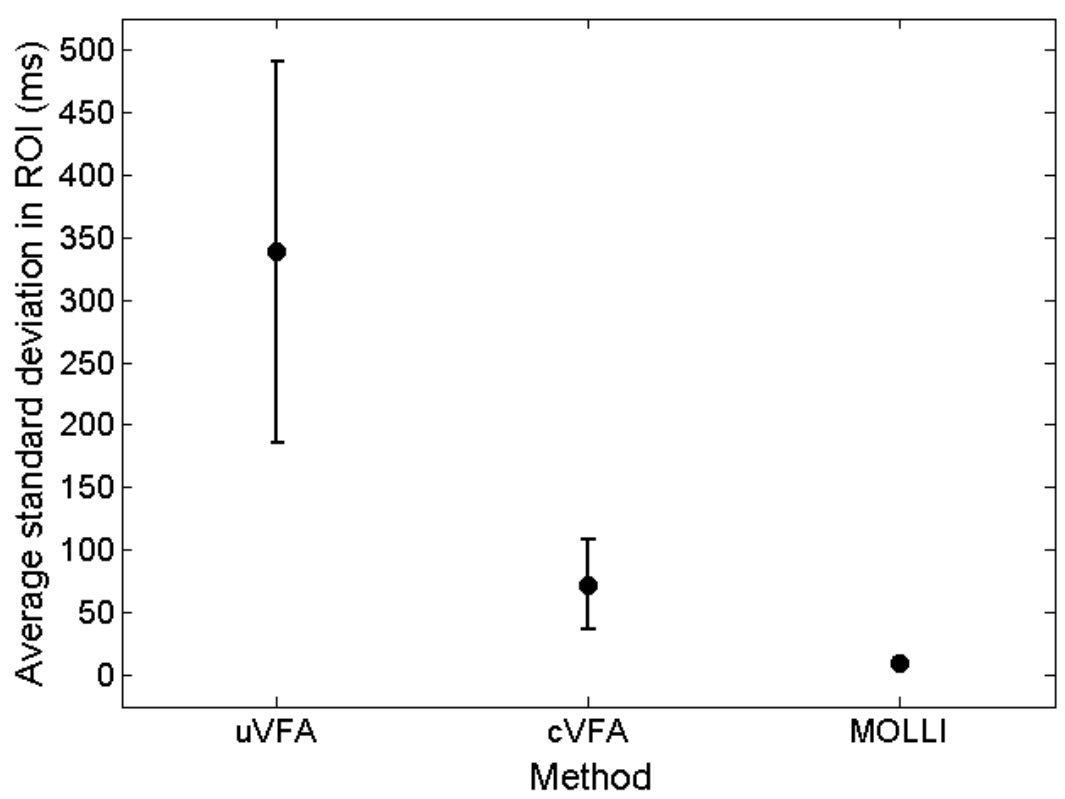

Figure 5.2: Average standard deviation for the three $T_{1}$ mapping methods.

repetition.

It should be made clear that the point of the thesis was not to find the best method for measuring $T_{1}$ but rather to use a good method, any good method, to study the reliability of the $T_{1 \text { pre }}$ and $\mathrm{BE}$ methods of determining $[G d]$. The MOLLI technique has been validated in this section as being a reliable method for measuring $T_{1}$.

\subsection{Accuracy, Precision, Spread, and Interslice Variation of $[G d]$ Results}

In order to further analyze the results presented in Section 4.3, a few figures of merit must first be defined. The "average slope" is simply the arithmetic mean of the four mean trendline slopes for each $[G d]$ quantification method seen in Figures 4.54.10. The average slope is an indication of the overall trend in accuracy for a given method. The "spread" of a given method is defined as the standard deviation of 
the reported mean trendline slopes. The spread reflects the reproducibility of each method with experiment repetition. The "precision" is a measure of the in-plane uniformity for a particular method. As explained earlier in Section 4.3, the reported minimum and maximum slopes are defined to be the slopes of the trends created by the negative and positive error bars, respectively, plotted against the reference values. The absolute difference between the minimum or maximum slope and the mean slope gives a representation of the overall precision for a single repetition of a given method. For instance, wide error bars, indicating non-uniform $[G d]$ maps, will trace out minimum and maximum trendlines that differ greatly from the central, mean trendline. Conversely, small error bars, indicating homogeneous $[G d]$ maps, will trace minimum and maximum trends that differ from the mean by very little. This is defined more formally in Eqs. (5.1) and (5.2) below.

With the above defined parameters, we may begin to look at the results of the experiments in more depth. The choice of $[G d]$ quantification method and the $T_{1}$ mapping technique used will greatly affect the accuracy, precision, and repeatability of $[G d]$ measurements. The purpose of the following sections is to quantify the changes observed in the measurement of $[G d]$ as a result of these options. Since all values are by nature normalized to a "true" slope of 1.00, the results may easily be interpreted as percent errors from the reference slope.

\subsubsection{Effect on Accuracy}

The accuracy of a given method represents how closely the $[G d]$ values within an ROI tend towards the true, reference $[G d]$ values. The results in Section 4.3 show that the accuracy of calculated $[G d]$ values is greatly influenced by both the $T_{1}$ mapping technique and the $[G d]$ quantification method used. The resulting average slopes for each method are given in Table 5.3 and presented visually in Figure 5.3. Recall that an average slope closer to 1.00 represents better agreement with reference values. The 


\begin{tabular}{cc} 
& Average Slope \\
\hline$T_{1 p r e}-\mathrm{uVFA}$ & 0.85 \\
\hline $\boldsymbol{T}_{1 p r e}-\mathrm{cVFA}$ & 1.06 \\
\hline $\boldsymbol{T}_{1 p r e}-\mathrm{LL}$ & 1.13 \\
\hline BE-uVFA & 0.79 \\
\hline BE-cVFA & 0.91 \\
\hline BE-LL & 0.95 \\
\hline
\end{tabular}

Table 5.3: Resulting average slopes for the results in Section 4.3. The values listed here reflect the overall accuracy of a given method.

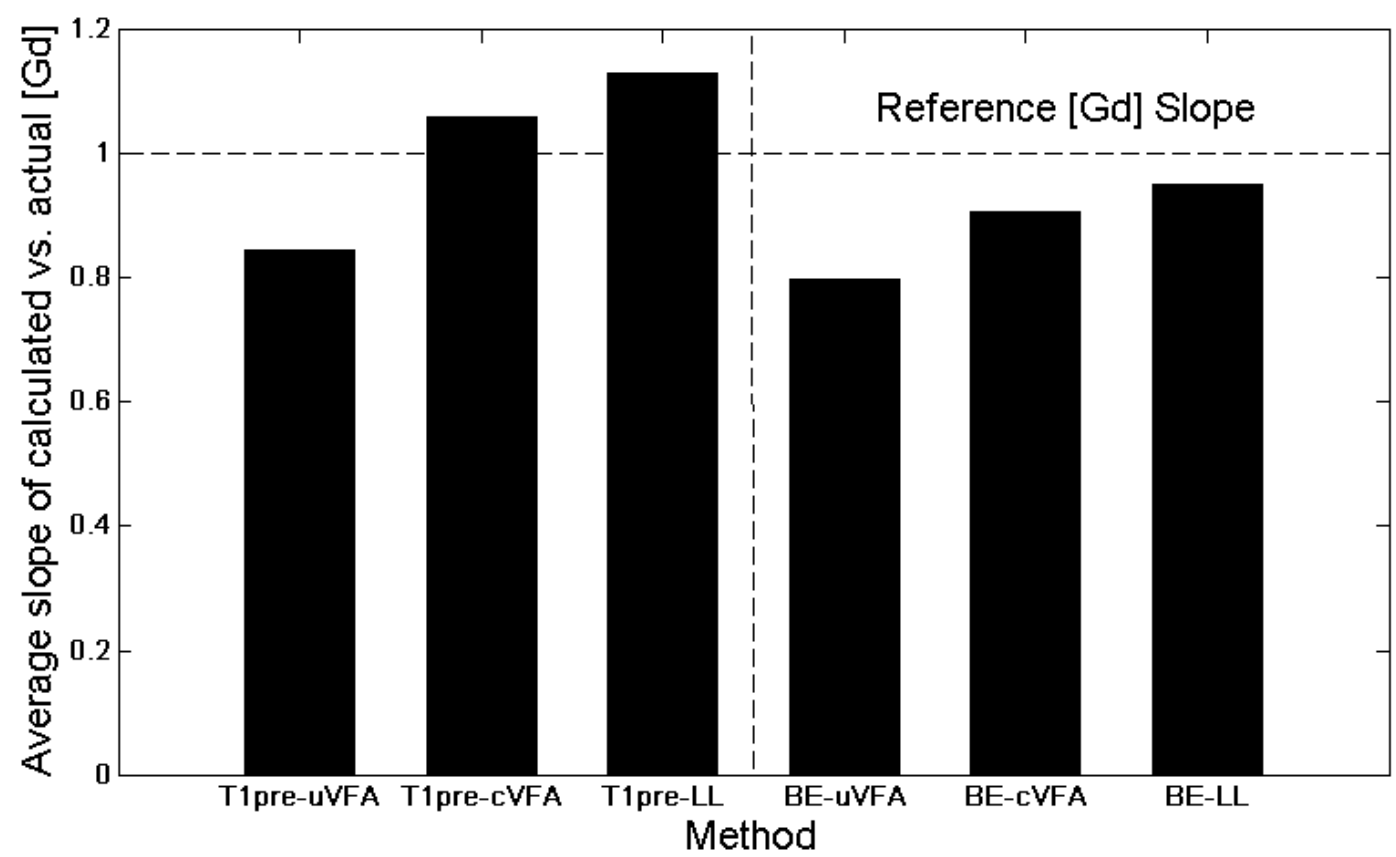

Figure 5.3: Trends observed in the slope of $[G d]$ quantification as a function of $T_{1}$ accuracy. The horizontal dashed line represents the ideal slope of 1.00 corresponding to perfect agreement with reference values. The vertical dashed line separates the $T_{1 \text { pre }}$ from the BE methods. 
general trend observed when using the Bookend method, and increasingly accurate $T_{1}$ maps, is convergence toward the true concentration values. The average slope increases from a low of 0.79 (BE-uVFA) to a high of 0.95 (BE-LL). This is because increasing $T_{1}$ accuracy allows for the better calibration of the signal strength equation, Eq. (2.27). Recall from Section 2.3.3 that calibration of the Bookend method requires two direct measurements of $T_{1}$ in order to estimate the calibration factor $P\left(T_{1}\right)$. With increased $T_{1}$ accuracy, calculations of $P_{\text {pre }}$ and $P_{\text {post }}$ become more accurate as well, allowing for a better estimate of $P\left(T_{1}\right)$ via equations Eqs. (2.30), (2.31), and (2.32) as a function of $T_{1}$. This type of behaviour is understandable and intuitive: as $T_{1}$ accuracy increases, so too does the accuracy of the $[G d]$ calculations. This is one of the great strengths of the Bookend method and a large contributor to the motivation behind its suggested use in the clinic.

Conversely, as is evident in Figure 5.3, the $T_{1 \text { pre }}$ method exhibits a different behaviour. In the case of using uVFA $T_{1}$ maps, the accuracy of the $T_{1 \text { pre }}$ method compared to the Bookend method is similar, with average slopes of 0.85 and 0.79 , respectively. However, with increasing $T_{1}$ accuracy it is observed that the $T_{1 \text { pre }}$ method trends toward overestimation of $[G d]$. The average slopes increase from a low of 0.85 $\left(T_{1 p r e}-\mathrm{uVFA}\right)$ to a high of $1.13\left(T_{1 p r e}-\mathrm{LL}\right)$. The reasons for this strange behaviour are two-fold.

Firstly, recall from Figure 2.7 that $P\left(T_{1}\right)$ is at its lowest value prior to injection of contrast agent and that this is the value used to calibrate the signal strength equation for every value of $T_{1}$. This means that the "calibrated" right hand side of the signal strength equation, Eq. (2.27), is always scaled down with respect to the true signal strength equation. This effect is simulated in Figure 5.4, which illustrates the relationship between $S$ and $T_{1}$ using the $T_{1 \text { pre }}$ method. This diagram shows that the improperly calibrated signal is a suppressed version of the true signal. As is evident, for the same values of signal strength on the y-axis, the improperly calibrated signal 
strength equation will return a lower value of $T_{1}$ than the true signal. Therefore, the

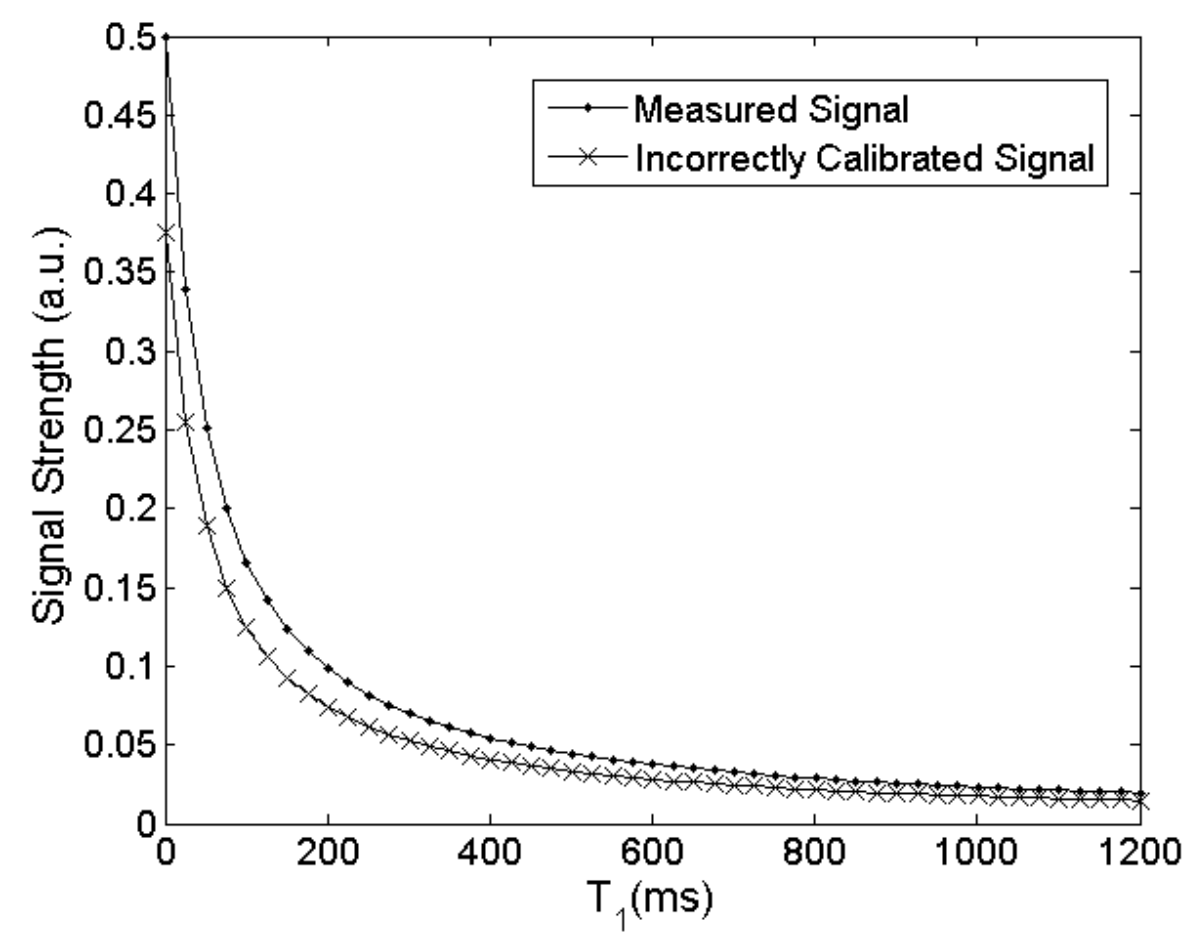

Figure 5.4: Simulated signal curves as a function of $T_{1}$. The incorrectly calibrated curve represents a scaled down version of the true measured signal by a factor of 0.75 . This value is arbitrary and was chosen for demonstration only. The curves were both generated with parameters used in the clinical DCE protocol. ( $\alpha=30$ degrees, $T R=6.5 \mathrm{~ms}$ ).

$T_{1 \text { pre }}$ method tends to consistently underestimate $T_{1}$. Due to the inverse relationship between $T_{1}$ and $[G d]$, as shown in Eq. (2.22), this corresponds to a consistent overestimation of $[G d]$, in agreement with the results reported in Figure 5.3.

Secondly, the determination of $P\left(T_{1}\right)$ prior to injection is heavily dependent on $T_{1}$ accuracy. Recall from Eq. (2.28), that $P_{\text {pre }}$ is defined as $P\left(T_{1 \text { pre }}\right)=S / F\left(\alpha, T R, T_{1 \text { pre }}\right)$. Inaccuracies in the measurement of $T_{1}$ will cause errors in the estimation of $P_{\text {pre }}$. In the case of VFA (both uncorrected and corrected), the tendency is for these methods is to overestimate $T_{1}$, as seen in Section 4.2.1. According to the shapes of the signal versus $T_{1}$ curves, as shown in Figure 5.4, an overestimation in $T_{1}$ will correspond to 
an underestimation in the theoretical signal strength, F. According to Eq. (2.28), this causes an artificial inflation of the calculated value of $P\left(T_{1}\right)$. Once again, referring to Figure 2.7, an artificially high calculated value of $P\left(T_{1}\right)$ corresponds to a better (although still rigidly constant over all $T_{1}$ 's) calibration of the signal strength equation. This will cause apparent increased $[G d]$ accuracy.

The two effects described counteract each other leading to values of $[G d]$ that are accidentally in agreement with the reference values. This is why, according to Figure 5.3, for intermediate $T_{1}$ accuracy (cVFA), the $T_{1 \text { pre }}$ agrees with reference far better than when $T_{1}$ accuracy is improved (MOLLI). This is a very significant problem with the $T_{1 \text { pre }}$ method, especially in the area of intermediate $T_{1}$ accuracy, because an increase in $T_{1}$ accuracy may counterintuitively lead to an apparent decrease in $[G d]$ accuracy. These results point out the unreliable and unpredictable nature of the $T_{1 \text { pre }}$ method when converting from signal strength to $[G d]$.

Note that the above explanation only holds within the context of the data collected for this work. In general, there is no a priori reason why $P\left(T_{1}\right)$ should decrease with $T_{1}$. It is possible for $P\left(T_{1}\right)$ to increase with $T_{1}$. In addition, the behaviour of the $T_{1 \text { pre }}$ method will change if $\mathrm{uVFA}$ and cVFA consistently underestimate, rather than overestimate, $T_{1}$. The preceding explanation is valid for the results presented in this work.

\subsubsection{Effect on Precision}

The overall precision of a given method is a reflection of the overarching homogeneity of the values observed within the ROI. Since the phantom is a water-filled jug with contrast agent added to manipulate $T_{1}$, and since measures were taken to ensure uniform distribution of the contrast agent within the volume, it is expected that the resultant concentration maps should be rather homogeneous. This is confirmed on the reference concentration maps provided by the gold standard TSE-IR measurements. 
In this section, the average precision for a given repetition of a particular method is defined as:

$$
\text { Precision }_{r e p}=1-\frac{\mid \text { max }- \text { mean }|+| \text { min }- \text { mean } \mid}{2},
$$

where mean represents the mean slope of the trend line being considered and $\min$ and max refer to the slope calculated for the negative and positive error bars, respectively. The overall precision for a given method is the difference from unity of the arithmetic mean of all four calculated precisions per repetition

$$
\text { Precision }=\left[\frac{\sum_{i=1}^{4} \text { Precision }_{r e p, i}}{4}\right]
$$

A method with a high precision as defined above represents good uniformity of $[G d]$ values within the concentration map. The precision values for the various methods considered in this work are reported in Table 5.4

\begin{tabular}{cc} 
& Precision \\
\hline $\boldsymbol{T}_{1 p r e}$-uVFA & 0.77 \\
\hline $\boldsymbol{T}_{1 p r e}$-cVFA & 0.89 \\
\hline $\boldsymbol{T}_{\text {1pre }}$-LL & 0.93 \\
\hline BE-uVFA & 0.78 \\
\hline BE-cVFA & 0.94 \\
\hline BE-LL & 0.98 \\
\hline
\end{tabular}

Table 5.4: Resulting precision for the results in Section 4.3. The values listed here reflect the overall uniformity of a given method.

A general trend for both the $T_{1 \text { pre }}$ and Bookend methods is quickly spotted. With 
increasing $T_{1}$ accuracy, both methods provide $[G d]$ results that are increasingly uniform. This most certainly stems from the increasing uniformity of the $T_{1}$ maps themselves, as indicated earlier in Section 5.1. However, it is also clear from Table 5.4 and Figure 5.5, that the Bookend method results are much more uniform than the $T_{1 \text { pre }}$ method. In the case of using inaccurate $\mathrm{UVFA}$ maps, both $T_{1 \text { pre }}$ and Bookend

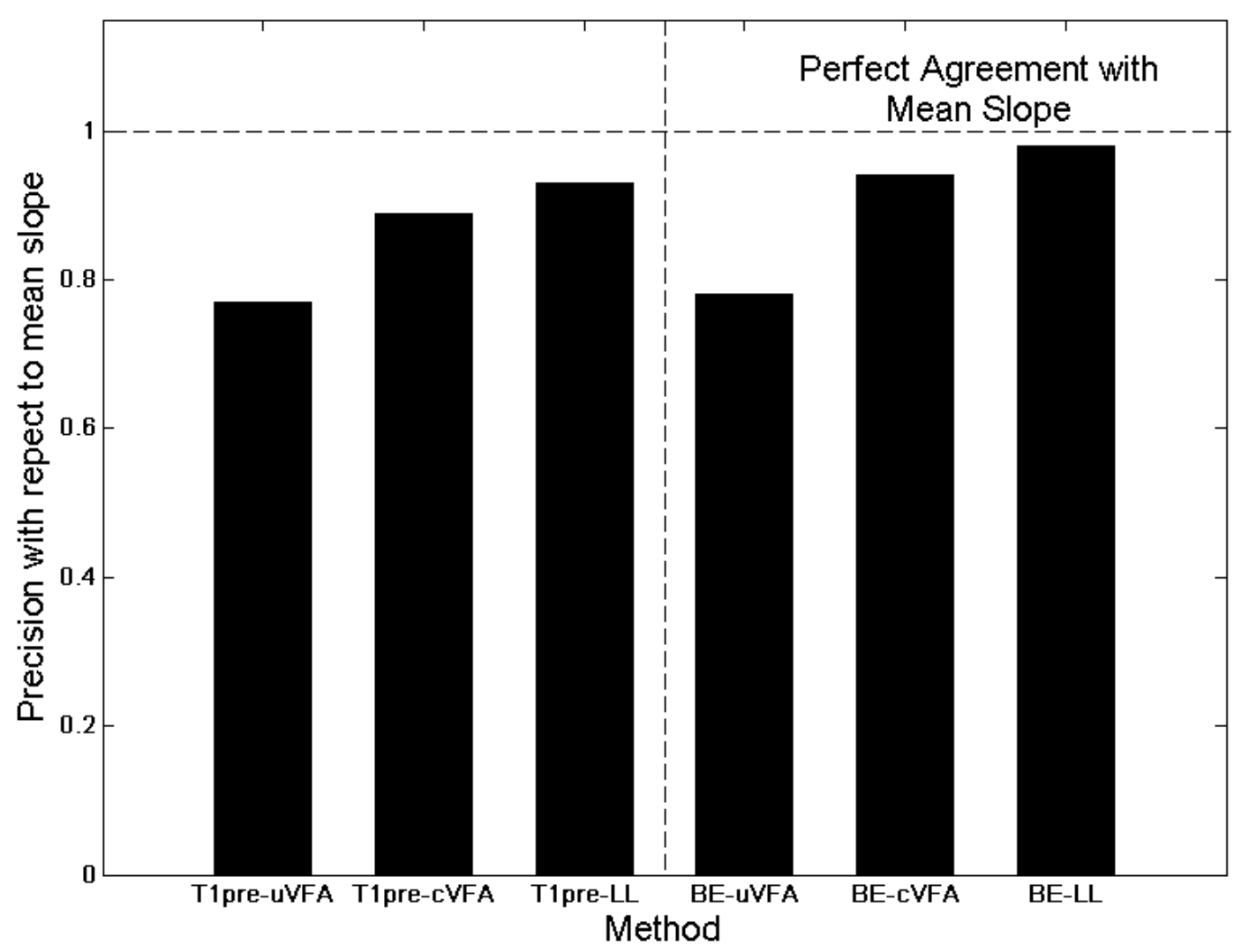

Figure 5.5: Trends in precision of the $T_{1 \text { pre }}$ and Bookend methods as a function of $T_{1}$ accuracy. The horizontal dashed line represents perfect agreement with the mean slope i.e. the min/max slopes do not differ from the mean. The vertical dashed line separates $T_{1 \text { pre }}$ from BE.

have similarly poor values of 0.77 and 0.78 , respectively. As $T_{1}$ accuracy improves, the Bookend values increase in uniformity relative to $T_{1 \text { pre }}$. When using cVFA maps, representing intermediate $T_{1}$ accuracy, the $T_{1 \text { pre }}$ method shows precision values that 
differ by $11 \%$ from the mean trend, while the Bookend results only differs by $6 \%$, almost half of its counterpart. At high $T_{1}$ accuracy, represented by the MOLLI $T_{1}$ maps, the precision acheived by $T_{1 p r e}$ differs from the mean by $7 \%$ while the Bookend method reduces this values to $2 \%$. The results of increasing precision are visibly apparent in Figure 5.6.

As explained above, the $T_{1}$ maps are used to correct for variations in the 3DFLASH signal strength in both the $T_{1 \text { pre }}$ and Bookend methods. The Bookend method accounts for variations in the signal as $T_{1}$ changes. However, since direct measurements of $T_{1}$ are required to perform the calibration, there is a limit to the Bookend methods abilities. Inaccurate $T_{1}$ mapping causes poor calibration of the signal strength equation, allowing calculated values of $[G d]$ to fluctuate wildly. This is why the BE-uVFA and $T_{1 p r e}-\mathrm{uVFA}$ methods produce similarly poor precisions. Accurate and uniform $T_{1}$ maps are required to fully take advantage of the benefits of the Bookend method to constrain the signal strength equation.

In terms of image uniformity for $[G d]$ evaluation, the Bookend method provides a useful improvement over the $T_{1 \text { pre }}$ method, especially at higher $T_{1}$ accuracy. This is significant because eliminating random or artifactual spatial variation of $[G d]$ within the image is very important for DCE-MRI as tumours or other microvasculature of interest may theoretically be located anywhere in the image.

\subsubsection{Effect on Spread}

The spread of the results for a given method reflects the repeatability of the method with time. It is represented by the differences between the mean slopes of the trends in Section 4.3, for an individual method. In this experiment, each method was repeated four times on different days, sometimes months apart, in order to characterize the variation of the results obtained for the $[G d]$ quantification methods considered. For the purposes of this section, the spread is defined quantitatively as the standard 


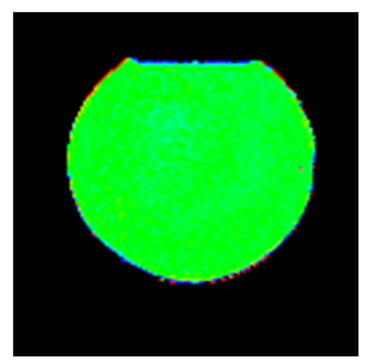

BE-LL

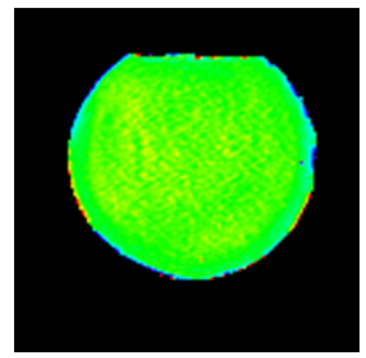

$\mathbf{T}_{1 \mathrm{pre}}-\mathbf{L L}$

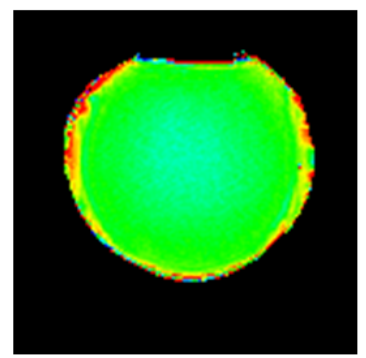

BE-cVFA

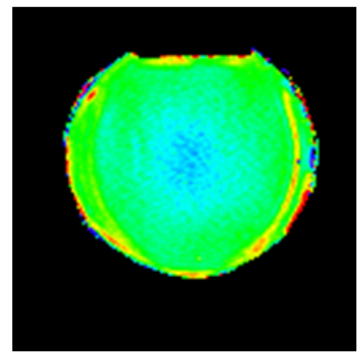

$\mathrm{T}_{1 \mathrm{pre}}{ }^{-\mathrm{cVFA}}$

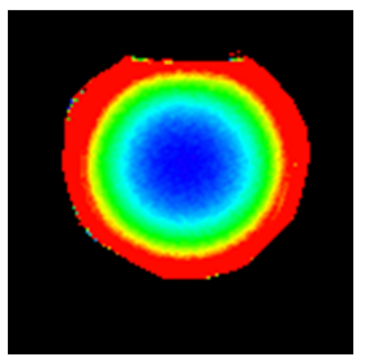

BE-UVFA

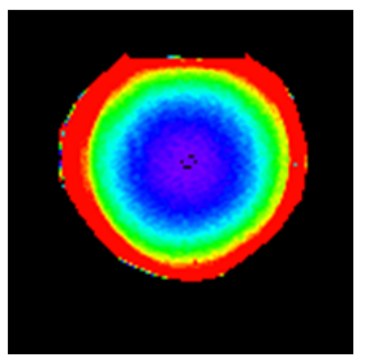

$T_{1 p r e}-\mathbf{U V F A}$
[Gd]
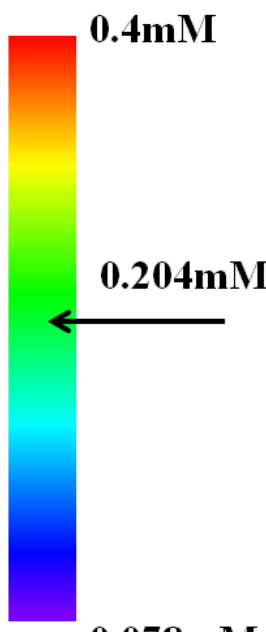

$0.078 \mathrm{mM}$

Figure 5.6: Images of the central $\left(9^{t h}\right)$ slice of the phantom for each $[G d]$ quantification method. The reference $[G d]$ is denoted by the arrow on the right. With increasing $T_{1}$ accuracy, images are noticeably more homogeneous. 


\begin{tabular}{cc} 
& Spread \\
\hline $\boldsymbol{T}_{1 p r e}$-uVFA & 0.12 \\
\hline $\boldsymbol{T}_{1 p r e}$-cVFA & 0.17 \\
\hline $\boldsymbol{T}_{1 p r e}$-LL & 0.12 \\
\hline BE-uVFA & 0.07 \\
\hline BE-cVFA & 0.04 \\
\hline BE-LL & 0.01 \\
\hline
\end{tabular}

Table 5.5: Resulting spread for the results in Section 4.3. The values listed here reflect the overall reproducibility of a given method.

deviation of the four mean slopes for a particular method reported in Section 4.3. A method with a low spread indicates the ability to provide highly repeatable results over time. The resulting calculations of the values of the spread for each method are found in Table 5.5. There is a stark and obvious difference between the $T_{1 \text { pre }}$ and Bookend methods. In the $T_{1 \text { pre }}$ case, increasing $T_{1}$ accuracy does not seem to have much of an effect on the resulting spread. This is consistent with the nature of the $T_{1 \text { pre }}$ methodology itself. Recall that in the $T_{1 \text { pre }}$ method, before the introduction of the Gd-based contrast agent, direct measurements of both $T_{1}$ and the average 3D-FLASH signal strength are performed. These measurements are then used to calculate the calibration factor $P_{\text {pre }}$ via Eq. (2.28), which is assumed to be valid for all values of $T_{1}$. It was shown, in Section 2.3.4 that this is not a correct assumption since $P\left(T_{1}\right)$ varies approximately linearly in $T_{1}$. The severity of the error introduced by this incorrect assumption is determined by the slope of $P\left(T_{1}\right)$. So, by increasing the accuracy of the $T_{1}$ measurements, $P\left(T_{1}\right)$ is more accurately calculated at that particular $T_{1}$ only. The trend of $P\left(T_{1}\right)$ (i.e. the slope) may vary from day to day due to RF miscalibration, changing $B_{1}$ profiles, etc. (see Section 2.3). A small 


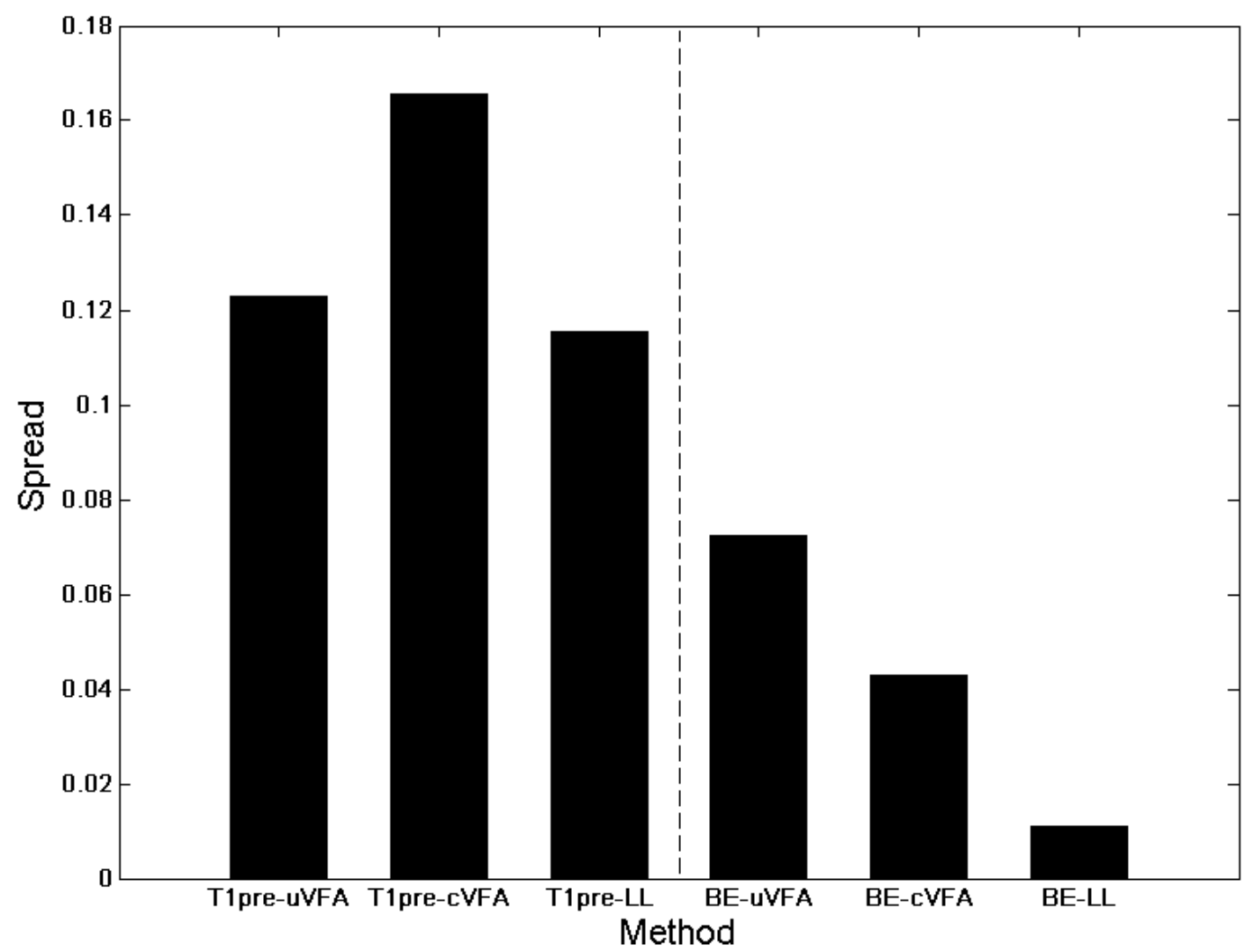

Figure 5.7: Trends in the spread for both the $T_{1 \text { pre }}$ and BE methods as a function of $T_{1}$ accuracy. The vertical dashed line separates $T_{1 \text { pre }}$ from BE.

slope represents little variation with $T_{1}$ and therefore the $T_{1 \text { pre }}$ method may produce reasonable results that agree well with reference values, as seen in several $T_{1 \text { pre }}$ cases. However, there is no guarantee that this trend in $P\left(T_{1}\right)$ will not change upon repetition of the experiment on another day. Even with the use of increasingly accurate $T_{1}$ maps, no steps are taken with the $T_{1 \text { pre }}$ method to account for variations in the slope of $P\left(T_{1}\right)$ between individual repetitions, which is why, as is corroborated by the results seen in Figure 5.7, $T_{1}$ accuracy seems to have no effect on the spread of results when using the $T_{1 \text { pre }}$ method. Even though the overall average accuracy may shift, the results show that the most accurate $T_{1 \text { pre }}$ case $\left(T_{1 \text { pre }}\right.$-LL) is likely to vary by as much as the most 
inaccurate $T_{1 \text { pre }}$ case $\left(T_{1 \text { pre }} \mathrm{uVA}\right)$ with spreads of 0.12 each. In fact, the intermediate case $\left(T_{1 p r e-c \mathrm{VFA}}\right)$ presents worse reproducibility than either of the other two, with a spread of 0.17 .

On the other hand, the Bookend method provides improved reproducibility as $T_{1}$ accuracy is improved. This trend is explained by the exact opposite argument used to describe the observed results for the $T_{1 \text { pre }}$ method. Recall that for the Bookend method, $T_{1}$ and signal strength are measured both before contrast agent injection and after the contrast agent concentration has plateaued. These are then used to approximate the linear relationship of $P\left(T_{1}\right)$. If $T_{1}$ is measured more accurately, the values of $P_{\text {pre }}$ and $P_{\text {post }}$ are calculated with greater confidence. This allows the linear fit of $P\left(T_{1}\right)$ to better represent the true nature of the 3D-FLASH signal offset as a function of $T_{1}$. Changes in the slope of $P\left(T_{1}\right)$ between individual repetitions of the experiment are accounted for with greater accuracy. In turn, this will provide more stable calibrations of the 3D-FLASH signal strength equation leading to increased reproducibility of $[G d]$ quantification. This is proven in the low spread values of the Bookend trendlines seen in Figure 5.7 and the decrease as $T_{1}$ accuracy improves. Even in the case of inaccurate $T_{1}$ maps (uVFA), using the Bookend method results in an approximately $40 \%$ reduction in the spread of results with repect to the corresponding $T_{1 \text { pre }}$ method (0.07 versus 0.12$)$.

In particular, the highly accurate BE-LL case illustrates significantly increased agreement between individual repetitions of the experiment with a spread of 0.01 . This represents an order of magnitude difference from any of the $T_{1 \text { pre }}$ cases. Therefore, the Bookend method results show significantly less day to day fluctuations than those obtained using the $T_{1 \text { pre }}$ method, even when $T_{1}$ accuracy is low. 


\subsubsection{Overall Effect}

In the above sections, observations of three different properties of the $[G d]$ quantification methods are analyzed. They are considered separately for simplicity. One aspect alone cannot be used to determine the overall quality of a particular $[G d]$ quantification method; all three properties must be considered when determining the overall quality of a particular method. For example, suppose one has access to intermediately accurate clinical $T_{1}$ mapping capabilities i.e. cVFA. According to Table 5.3, the overall accuracy of $T_{1 p r e} e^{-c V F A}$ is 1.06 while the overall accuracy of BE-cVFA is 0.91. This represents an overall, absolute percent error of $6 \%$ for the $T_{1 \text { pre }}$ method and $9 \%$ for the Bookend method. These values are fairly similar, but at first glance, it appears that the $T_{1 \text { pre }}$ method is superior to the Bookend method for $[G d]$ estimation. It is not until one investigates further that it becomes evident that this is not the case. Still considering the cVFA case, it is obvious that the average slope for a given method is not a good representation of its overall accuracy if the spread and precision are large, as is the case for the $T_{1 \text { pre }}$-cVFA method. The average slope only represents the centre of the cluster of trendlines produced in the four repetitions of the experiment. In this case, the average slope is centred on 1.06 which represents good agreement with reference values, but the trends reported in Section 4.3 and the calculated spread reported in Table 5.5 indicate that the measurements provided by the $T_{1 \text { pre }}$-cVFA method may vary by up to $\pm 17 \%$. This is a large amount of drift and unacceptable for clinical DCE-MRI experiments. Conversely, for the Bookend method, this variance in the trends is reduced to $\pm 4 \%$ for BE-cVFA. In addition to this, Table 5.4 indicates a higher degree of non-uniformity in the $T_{1 \text { pre }}$ case as opposed to the Bookend case (0.89 versus 0.94). Thus, it is clear in this case that the Bookend method provides more reliable $[G d]$ quantification when all aspects are considered. See Figure 5.8 for a visual representation of the trends. This figure amalgamates all 
three aspects coherently such that the true ability of each method to evaluate $[G d]$ becomes clear.

From this figure, it is apparent that the Bookend method provides more reasonable $[G d]$ quantification results than the $T_{1 \text { pre }}$ method. In terms of overall accuracy, the Bookend method consistently improves $[G d]$ quantification with increasing $T_{1}$ accuracy. The $T_{1 \text { pre }}$ method tends to overestimate $[G d]$ at intermediate and high levels of $T_{1}$ accuracy. In terms of image uniformity, both the Bookend and the $T_{1 \text { pre }}$ method provide more homogeneous maps has $T_{1}$ accuracy is improved. However, for the same level of $T_{1}$ accuracy, the former will produce considerably more uniform $[G d]$ maps. And finally, in terms of repeatability, the $T_{1 \text { pre }}$ method provides no discernible increase in reproducibility over time as $T_{1}$ accuracy is increased. Conversely, the Bookend method becomes extremely stable as more accurate $T_{1}$ maps are used.

\subsubsection{Through Plane Variation}

The results presented in Section 5.2.5 indicate that choice of $[G d]$ quantification method and $T_{1}$ mapping accuracy greatly affect the through plane concentration homogeneity. For the results shown in Tables 4.16 and 4.17 , the overall, average percent error for the $T_{1 \text { pre }}$ and Bookend methods were calculated to give an overarching representation of the through plane homogeneity. The standard deviation was also calculated to determine the variation in the percent error from slice to slice. The results of these calculations may be seen in Table 5.6 and Figure 5.9. For the Bookend method, the average percent error consistently decreases as $T_{1}$ accuracy improves, culminating with an average percent error of $-0.5 \%$ between slices for the high $T_{1}$ accuracy case (BE-LL). It is also observed that the variations in percent error between slices with respect to the central slice decrease rapidly with increasing $T_{1}$ accuracy from a high of $7.2 \%$ to a low of $1.5 \%$.

For the $T_{1 \text { pre }}$ method, the trend in the results is a little less clear. At first, glance it 


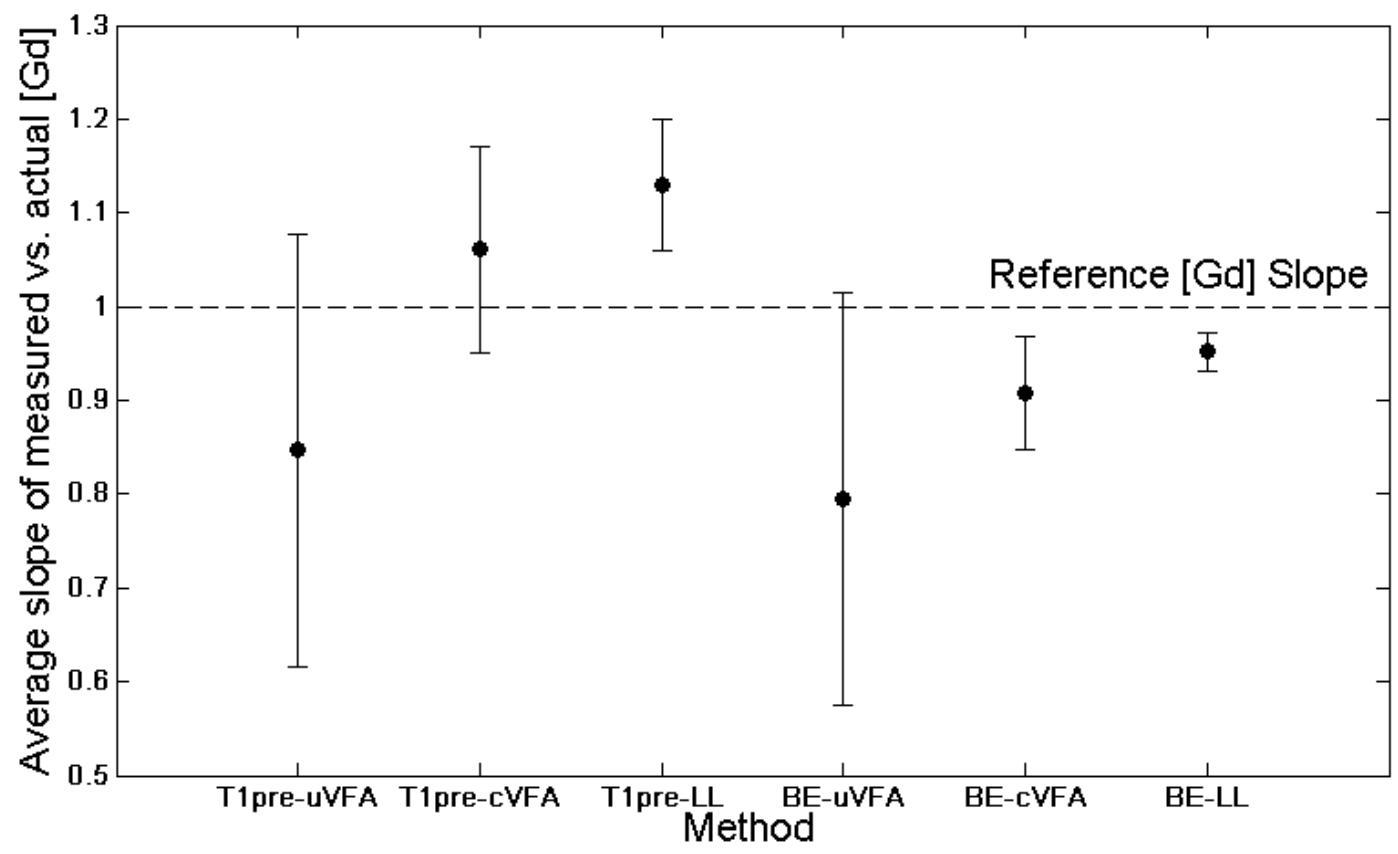

(a) Figure illustrating the trends of the average slope for all methods. Positive and negative error bars represent 1-Precision values calculated and presented in Section 5.2.2.

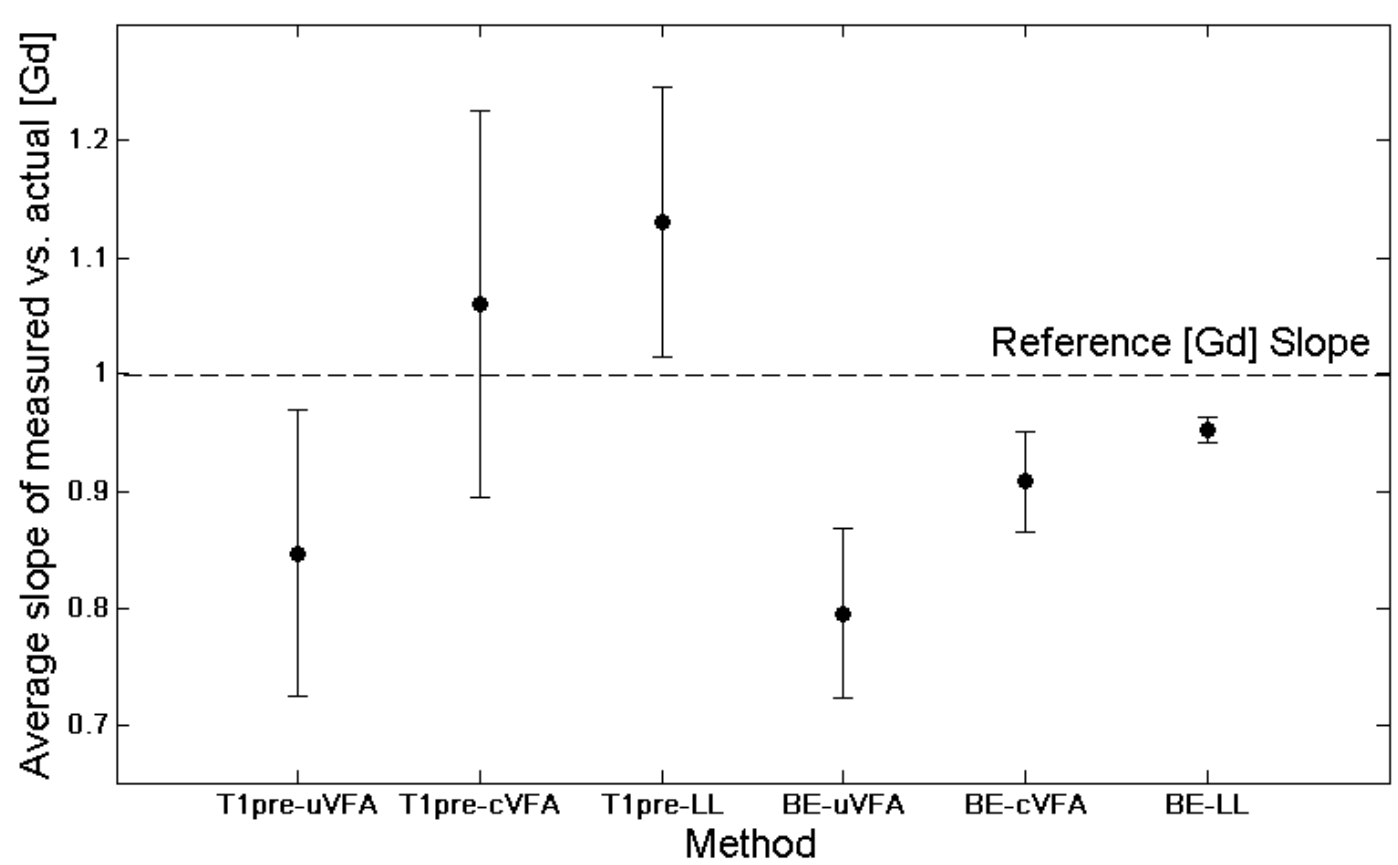

(b) Figure illustrating trends of average slope for all methods. Positive and negative error bars represent the Spread values as calculated and presented in Section 5.2.3.

Figure 5.8: The average slopes along with 1-Precision (a) and Spread (b) as error bars. (a) represents the overall accuracy and image uniformity for a given method, while (b) represents the overall accuracy and reproducibility of results for a given method. 


\begin{tabular}{cc} 
& Average Percent Error \\
\hline $\boldsymbol{T}_{1 p r e}-\mathrm{uVFA}$ & $-0.6 \pm 10.7$ \\
\hline $\boldsymbol{T}_{1 p r e}-\mathrm{cVFA}$ & $-4.7 \pm 5.4$ \\
\hline $\boldsymbol{T}_{1 p r e}-\mathrm{LL}$ & $-0.1 \pm 7.6$ \\
\hline BE-uVFA & $5.9 \pm 7.2$ \\
\hline BE-cVFA & $3.1 \pm 2.9$ \\
\hline BE-LL & $-0.5 \pm 1.5$ \\
\hline
\end{tabular}

Table 5.6: The average percent error with respect to the central slice for all $[G d]$ quantification methods. The errors represent the standard deviation of reported percent errors across slices.

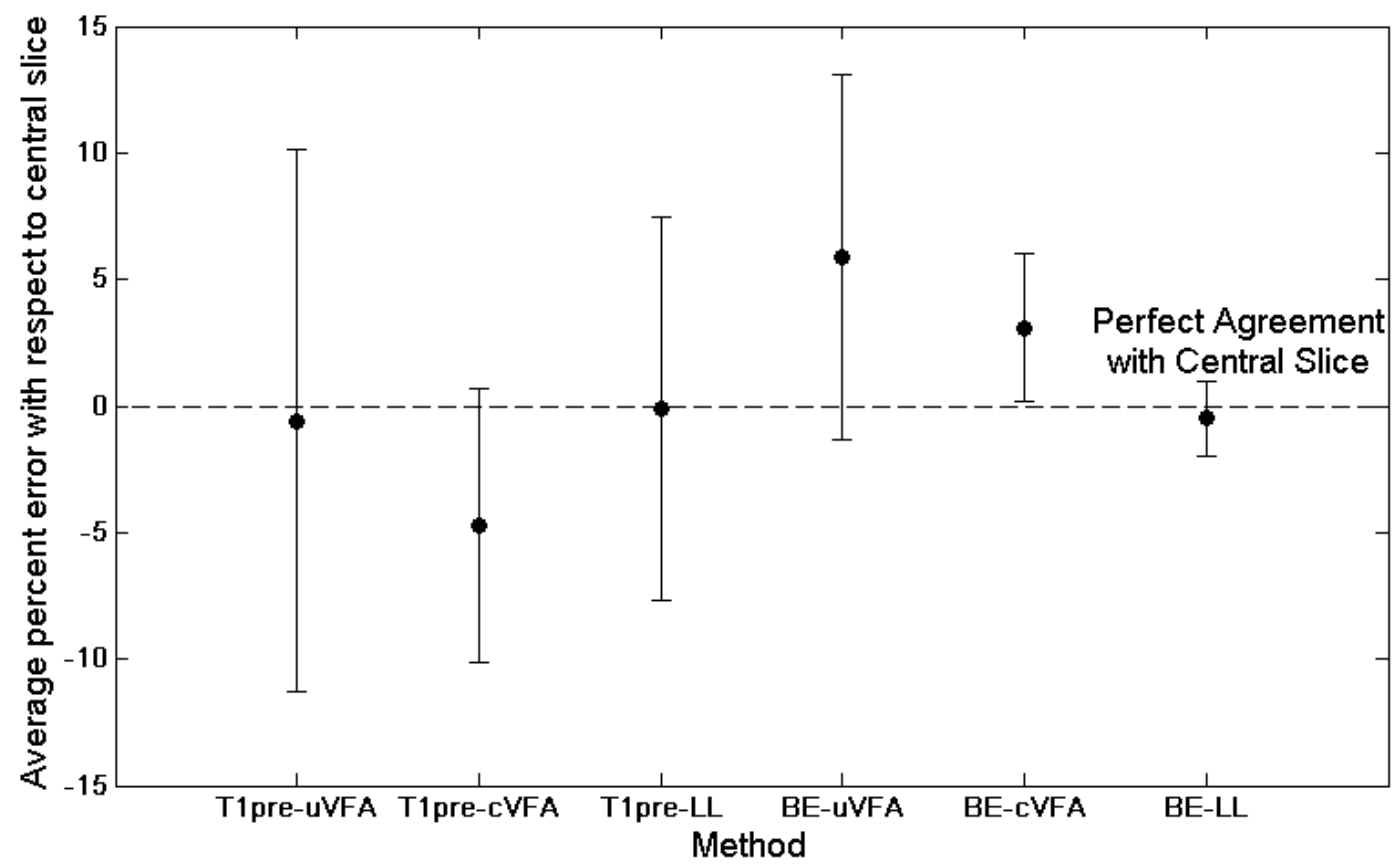

Figure 5.9: Uniformity of $[G d]$ quantification for each method. Error bars represent standard deviation of reported percent errors with respect to the central slice. Horizontal dashed line represents perfect agreement with the central slice. 
appears that the $T_{1 p r e}$-based techniques grant better through plane accuracy than the Bookend methods. The average percent errors for the uVFA and LL based methods yield extremely low average percent errors of $-0.6 \%$ and $-0.1 \%$, respectively. However, as is denoted by the large errors, these methods tended to vary wildly about the reference slice. The averages calculated are close to reference simply because the variations above and below reference seem to be evenly distributed. Strangely, in the intermediate accuracy case (cVFA) the average percent error increased to $-4.7 \%$. In addition, it appears that increasing $T_{1}$ accuracy does not seem to affect the variations in percent error from slice to slice. In the low $T_{1}$ accuracy case $\left(T_{1 p r e} \mathrm{uVFA}\right)$ the standard deviation of the reported percent error is $10.7 \%$, while the high $T_{1}$ accuracy case $\left(T_{1 p r e}-\mathrm{LL}\right)$ has a value of $7.6 \%$. In fact, the intermediate accuracy case provided the least amount of variation between slices with a value of $5.4 \%$.

Once again, this illustrates the strange and unreliable nature of the $T_{1 \text { pre }}$ method since interslice variations seem to be unaffected by increasing $T_{1}$ accuracy. On the other hand, the Bookend method consistently improves interslice uniformity as $T_{1}$ accuracy is improved.

A similar analysis was also performed to evaluate the trends in image homogeneity for each of the six methods as a function of slice number. The errors reported in Tables 4.16 and 4.17 represent the image uniformity tracked as a function of slice number. The overall average and standard deviation of these results were calculated for each method to determine how the overall precision varied throughout the imaging volume. The results of these calculations are presented in Table 5.7 and Figure 5.10. As a function of slice number, it is observed that, for both the $T_{1 p r e}$ and Bookend methods, image uniformity consistently increases as $T_{1}$ accuracy increases. This was expected due to the results shown in Section 5.2.2. Mimicking those results, it is seen that, even though both methods improve with increasing $T_{1}$ accuracy, ultimately it is the Bookend method that provides the most uniform maps throughout the volume. 


\section{Average Precision}

\begin{tabular}{cc}
\hline $\boldsymbol{T}_{\text {1pre }}$-uVFA & $30.4 \pm 1.0$ \\
\hline $\boldsymbol{T}_{\text {1pre }}$-cVFA & $9.4 \pm 1.9$ \\
\hline $\boldsymbol{T}_{\text {1pre }}$-LL & $6.2 \pm 1.2$ \\
\hline BE-uVFA & $27.7 \pm 1.0$ \\
\hline BE-cVFA & $5.6 \pm 0.3$ \\
\hline BE-LL & $3.6 \pm 0.3$ \\
\hline
\end{tabular}

Table 5.7: The average precision for all $[G d]$ quantification methods. The errors represents the standard deviation of reported percent errors calculated with respect to the central slice.

In the case of inaccurate $T_{1}$ maps $(\mathrm{uVFA})$ the Bookend and $T_{1 \text { pre }}$ methods have a similar average precision of $30.4 \%$ and $27.7 \%$, respectively. However for the LL case, the Bookend method quickly outperforms the $T_{1 \text { pre }}$ method in image uniformity with average precisions of $3.6 \%$ versus $6.2 \%$, respectively.

A more significant trend is spotted in the errors, which represent the standard deviations of the precisions reported in Section 5.2.5. While both methods tend not to vary much between slices, the $T_{1 \text { pre }}$ method holds steady around $1 \%$, increasing to $1.9 \%$ in the $T_{1 p r e}$-cVFA case. On the other hand, for the Bookend method, there is a decrease between the uVFA and cVFA cases from $1 \%$ to $0.3 \%$. The Bookend method then holds steady at $0.3 \%$ for the LL case. Overall, however, both methods appear to be stable in terms of interslice variations in the image uniformity, varying at most by $2 \%$.

Once again, the Bookend method provides significant advantages over the $T_{1 \text { pre }}$ method. In terms of through plane homogeneity, Figures 5.9 and 5.10 indicate that the Bookend method reduces variations in $[G d]$ quantification accuracy and precision when increasingly accurate $T_{1}$ maps are employed. 


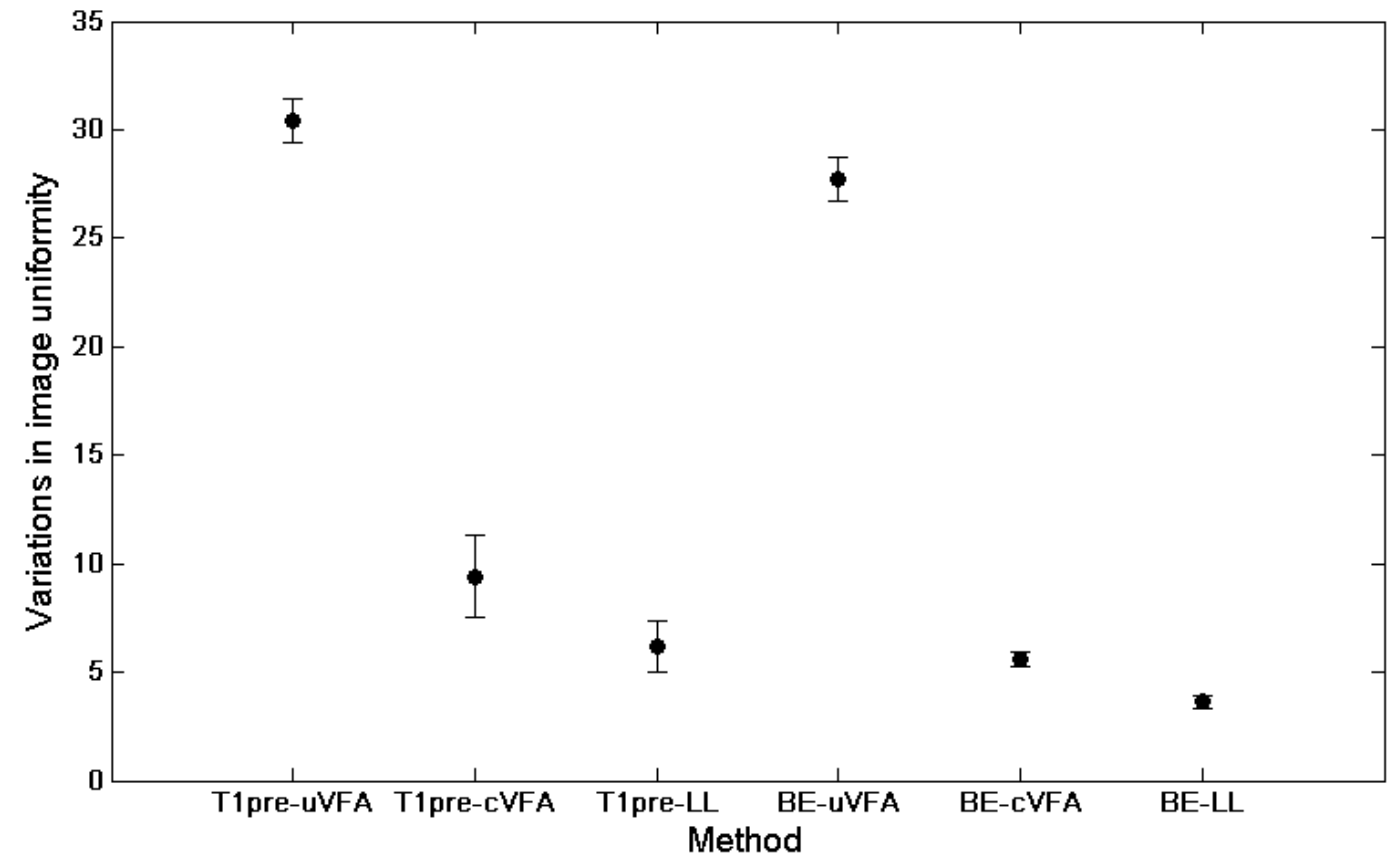

Figure 5.10: Average interslice image uniformity for each $[G d]$ quantification method. Error bars represent the standard deviation of reported percent errors with respect to the central slice. 
In conclusion, the overall performance of the BE-LL method is far superior to any of the other methods considered. In particular, the commonly used $T_{1 \text { pre }}$ method performed quite poorly compared with the Bookend method, especially when accurate $T_{1}$ maps were used. 


\section{Chapter 6}

\section{Conclusion}

\subsection{Final Comments: The Bookend Method ver- sus The $T_{1 p r e}$ Method}

The results presented in Chapters 4 and 5 have unquestionably illustrated the advantages of the Bookend method over the $T_{1 \text { pre }}$ method for the purposes of $[G d]$ quantification at $3 \mathrm{~T}$. The results also show the behaviour of both methods as input $T_{1}$ accuracy is varied. These results are significant because, at the moment, measurements performed using the $T_{1 \text { pre }}$ method are, unfortunately, the most prevalent form of $[G d]$ quantification used clinically. Therefore, it is with great hope that the results seen in this work may be put to good use to increase the quantitative accuracy of DCE-MRI. In fact, based on the observations reported in this work, the clinical protocol at The Ottawa Hospital has since been amended to include MOLLI measurements of $T_{1 \text { pre }}$ and $T_{1 \text { post }}$ with the goal of improving in vivo $T_{1}$ accuracy and increasing the quantitative reliability of $[G d]$ measurements in clinical DCE-MRI studies. As mentioned in Sections 2.2.2 and 2.2.3, some methods for determining the vascular input of contrast agent, the AIF, use measurements made directly from calculated $[G d]$ maps. In studies where this is performed, this is an extra boon, since increasing 
overall quantitative accuracy of $[G d]$ will also aid in the improvement of AIF accuracy.

To reiterate and summarize the results:

1. In Section 5.2.1, it was clearly observed that overall accuracy of $[G d]$ quantification consistently improved as increasingly accurate $T_{1}$ maps were used in conjunction with the Bookend method. This was not the case with the $T_{1 p r e}$ method. Increasing $T_{1}$ accuracy prompted the $T_{1 \text { pre }}$ method to overestimate $[G d]$, highlighting the unreliable nature of the method.

2. In Section 5.2.2, the effect on precision for both methods was an observed increase in image uniformity as $T_{1}$ accuracy improves. Both methods provide images that are unambiguously more homogeneous when input $T_{1}$ maps are accurate. This is without a doubt largely due to the increasing uniformity of the $T_{1}$ maps themselves. However, it was noticed that the images produced using the Bookend method had increased precision with respect to the $T_{1 \text { pre }}$ method for the same level of $T_{1}$ accuracy.

3. In Section 5.2.3, the spread of the resulting $[G d]$ trendlines presented in Section 4.3 diminished rapidly with increasingly accuracy $T_{1}$ maps for the Bookend method. Each Bookend-based method consistently became more stable with repeated experiments as $T_{1}$ accuracy improved. Conversely, increasing $T_{1}$ accuracy did not seem to affect the resulting spread of the trendlines for the $T_{1 \text { pre }}$ method. In fact, an increase in spread was observed when $T_{1}$ maps of intermediate accuracy were employed.

4. In Section 5.2.5, in terms of through plane homogeneity, it was identified that there are considerable advantages when using the Bookend method. Average percent differences with respect to the central slice of the phantom, were seen to be increasingly in agreement and more stable as $T_{1}$ accuracy increased. On 
the other hand, the $T_{1 \text { pre }}$ was observed to vary significantly between slices, even with improved $T_{1}$ accuracy. In terms of image uniformity between slices, both methods were seen to improve substantially. However, the Bookend method had the greater improvement and less interslice variations in image uniformity were observed.

\subsection{Limitations of this work}

The work presented here is not an exhaustive investigation into the effect of using the Bookend method to conduct DCE-MRI experiments. As explained in Sections 2.2.2 and 2.2.3, $[G d]$ quantification is only one of several necessary elements in order to produce parametric maps of biologically relevant kinetic parameters. The effects of employing the superior $[G d]$ evaluation, seen in this work, into the pharmacokinetic modelling were not investigated.

In addition, as explained in Section 3.3.1 and 4.1, inversion recovery based $T_{1}$ measurements were used as the sole reference value. As previously alluded to [41,42], it has been shown that IR is a reliable method for mapping the longitudinal relaxation and that the equation governing the relationship between $T_{1}$ and $[G d]$ (Eq. (2.22)) has been validated to hold true over a wide concentration range. However, no attempt was made to measure the contrast agent concentration directly. There are likely deviations between the $[G d]$ predicted strictly through measurement of $T_{1}$ and the true $[G d]$, though the literature suggests that these differences are small [66].

An important parameter for the calculation of concentration using Eq. (2.22) is the relaxivity, $r_{1}$, of the contrast agent. $r_{1}$ is known to vary with temperature $[22,26]$. A value of $r_{1}$ near body temperature was assumed. However, when the phantom was filled with water, efforts were made to have the temperature of the water near room 
temperature. This was done so there would not be a change in temperature (therefore a change in $r_{1}$ ) with time as the imaging experiment progressed, as the water temperature could not be manipulated during imaging. This may have corresponded to an underestimation of the value of $r_{1}$. According to Eq. (2.22) this would lead to a systematic overestimation of $[G d]$. However, assuming no radical temperature fluctuations throughout the imaging experiment, even though this may affect the absolute value of the quantification of $[G d]$, this should not affect the trends in quantitative accuracy observed, since all concentration quantification methods would have been affected by the same factor.

Experiments for each of the six resultant $[G d]$ evaluation methods in this work were repeated four times on different days to assess the overall quantitative accuracy and reproducibility over time. Unfortunately the time between experiments for several methods varied greatly. Repetitions of the experiments for the $T_{1 \text { pre }}-\mathrm{uVFA}, T_{1 \text { pre }}{ }^{-}$ cVFA, BE-uVFA, and BE-cVFA methods were conducted between October 2012 and June 2013, often allowing for months in between experiments. However, for the MOLLI based methods ( $T_{1 p r e}-\mathrm{LL}$ and BE-LL), the timeframe between experiments was greatly reduced and the experiments were only conducted between March 2013 and June 2013 with 3 of the 4 experiments being conduced withing the span of a month. This was because the MOLLI $T_{1}$ mapping sequence was not acquired until late March 2013. Although likely very small, this quick repetition may cause a bias in the MOLLI based measurements of $[G d]$.

\subsection{Future Work}

An obvious step for future work is the application of the results observed in this work to in vivo clinical trials. By adding a second, accurate $T_{1}$ measurement $\left(T_{1 \text { post }}\right)$ to clinical protocols, systematic comparison between the Bookend and $T_{1 \text { pre }}$ methods 
could be performed with human subjects.

As mentioned above in Section 6.2, the effect of $[G d]$ quantification accuracy on the output of the pharmacokinetic modelling was not investigated. This is a crucial area of DCE-MRI that requires investigation. The output pharmacokinetic parameters are the end result of DCE-MRI procedures that are of interest to clinicians. The effect of $[G d]$ accuracy on pharmacokinetic modelling needs to be investigated and understood.

By nature, the total $T_{1}$ mapping time required to incorporate Bookend $[G d]$ quantification is twice as long as the $T_{1 p r e}$ method. This is why a clinical time threshold of 3 minutes per $T_{1}$ map was used as criteria for selecting a $T_{1}$ measurement technique. Some DCE-MRI procedures or other clinics may require a lower threshold than this. Therefore, methods that decrease acquisition time, while maintaining a high level of accuracy are, as ever, in high demand. This need for fast acquisition speed is more pronounced when the Bookend method is employed. However, as the results of this work show, the benefits appear to be well worth it. 


\section{List of References}

[1] I. Rabi, J. Zacharias, S. Millman, and P. Kusch. "A New Method of Measuring Nuclear Magnetic Moment." Physical Review 53(4), 318-327 (1938).

[2] F. Bloch, W. Hansen, and M. Packard. "The Nuclear Induction Experiment." Physical Review 70(7,8), 474-485 (1946).

[3] E. Purcell, H. Torrey, and R. Pound. "Resonance Absorption by Nuclear Magnetic Moments in a Solid." Physical Review 69(1,2), 37-38 (1946).

[4] P. Lauterbur. "Image Formation by Induced Local Interactions: Examples Employing Nuclear Magnetic Resonance." Nature 242, 190-191 (1973).

[5] A. Jackson, D. L. Buckley, and G. J. M. Parker. Dynamic Contrast Enhanced Magnetic Resonance Imaging in Oncology. Springer, New York (2005).

[6] A. Rescigno and A. Boicelli. Cerebral Blood Flow: Mathematical Models, Intrumentation, and Imaging Techniques. Plenum Press, New York and London (1988).

[7] R. M. Weisskoff, D. Cheder, J. L. Boxerman, and B. R. Rosen. "Pitfalls in MR Measurement of Tissue Blood Flow with Intravascular Tracers: Which Mean Transit Time." Magnetic Resonance in Medicine 29(4), 553-558 (1993).

[8] J. E. Perthen, F. Calamante, D. G. Gadian, and A. Connelly. "Is Quantification of Bolus Tracking MRI Reliable Without Deconvolution?" Magnetic Resonance in Medicine 67(1), 61-67 (2002).

[9] D. Griffiths. Introduction to Quantum Mechanics. Pearson Prentice-Hall, Upper Saddle River, NJ, 2nd edition (2004).

[10] J. Prince and J. Links. Medical Imaging Signals and Systems. Pearson PrenticeHall, Upper Saddle River, NJ (2006). 
[11] Z. Liang and P. Lauterbur. Principles of Magnetic Resonance Imaging: A Signal Processing Perspective. IEEE Press, Piscataway, NJ (2000).

[12] E. M. Haacke, R. W. Brown, M. R. Thompson, and R. Venkatesan. Magnetic Resonance Imaging: Physical Principles and Seuqence Design. Wiley (1999).

[13] O. Gerlach and W. Stern. "Das magnetische Moment des Silberatoms." Zeitshcrift fur Physik 9(1), 353-355 (1922).

[14] F. Reif. Fundamentals of Statistical and Thermal Physics. Waveland Press (2009).

[15] M. J. Paldino and D. P. Barboriak. "Fundamentals of Quantitative Dynamic Contrast-Enhanced MR Imaging." Magnetic Resonance Imaging Clinics of North America 17(2), 277-289. ISSN 1064-9689 (2009).

[16] A. R. Padhani. "Dynamic contrast-enhanced MRI in clinical oncology: Current status and future directions." Journal of Magnetic Resonance Imaging 16(4), 407-422. ISSN 1522-2586 (2002).

[17] P. L. Choyke, A. J. Dwyer, and M. V. Knopp. "Functional tumor imaging with dynamic contrast-enhanced magnetic resonance imaging." Journal of Magnetic Resonance Imaging 17(5), 509-20. ISSN 1053-1807 (2003).

[18] A. Jackson, J. P. B. O’Connor, G. J. M. Parker, and G. C. Jayson. "Imaging tumor vascular heterogeneity and angiogenesis using dynamic contrast-enhanced magnetic resonance imaging." Clinical Cancer Research 13(12), 3449-59. ISSN 1078-0432 (2007).

[19] C. Hayes, A. R. Padhani, and M. O. Leach. "Assessing changes in tumour vascular function using dynamic contrast-enhanced magnetic resonance imaging." NMR in Biomedicine 15(2), 154-163 (2002).

[20] A. R. Padhani and M. O. Leach. "Antivascular cancer treatments: functional assessments by dynamic contrast-enhanced magnetic resonance imaging." $A b$ dominal Imaging 30(3), 324-41. ISSN 0942-8925 (2005).

[21] T. B. Nguyen, G. O. Cron, J. F. Mercier, C. Foottit, C. H. Torres, S. Chakraborty, J. Woulfe, G. H. Jansen, J. M. Caudrelier, J. Sinclair, M. J. Hogan, R. E. Thornhill, and I. G. Cameron. "Diagnostic accuracy of dynamic contrast-enhanced MR imaging using a phase-derived vascular input function in the preoperative grading of gliomas." American Journal of Neuroradiology 33(8), 1539-45. ISSN 1936-959X (2012). 
[22] S. Laurent, L. V. Elst, and R. N. Muller. "Comparative study of the physicochemical properties of six clinical low molecular weight gadolinium contrast agents." Contrast Media \& Molecular Imaging 1(3), 128-37. ISSN 1555-4317 (2006).

[23] P. Caravan, J. J. Ellison, T. J. McMurry, and R. B. Lauffer. "Gadolinium(III) Chelates as MRI Contrast Agents: Structure, Dynamics, and Applications." Chemical reviews 99(9), 2293-352. ISSN 1520-6890 (1999).

[24] S. H. Koenig, M. Spiller, R. D. Brown, and G. L. Wolf. "Relaxation of Water protons in the Intra- and Extracellular Regions of Blood Bontaining Gd(DTPA)." Magnetic Resonance in Medicine 3(5), 791-5. ISSN 0740-3194 (1986).

[25] M. F. Tweedle, P. Wedeking, J. Telser, C. H. Sotak, C. a. Chang, K. Kumar, X. Wan, and S. M. Eaton. "Dependence of MR Signal Intensity on Gd Tissue Concentration over a Broad Dose Range." Magnetic Resonance in Medicine 22(2), 191-4. ISSN 0740-3194 (1991).

[26] J. Pintaske, P. Martirosian, H. Graf, G. Erb, K. Lodeman, C. Claussen, and F. Schick. "Relaxivity of Gadopentetate Dimeglumine (Magnevist), Gadobutrol (Gadovist), and Gadobenate Dimeglumine (MultiHance) in Human Blood Plasma at 0.2, 1.5, and 3 Tesla." Investigative Radiology 41(3), 213-221 (2006).

[27] P. S. Tofts, G. Brix, D. L. Buckley, J. L. Evelhoch, E. Henderson, M. V. Knopp, H. B. W. Larsson, T. Y. Lee, N. A. Mayr, G. J. M. Parker, R. E. Port, J. Taylor, and R. M. Weisskoff. "Contrast-Enhanced T1-Weighted MRI of a Diffusable Tracer: Standardized Quantities and Symbols." Journal of Magnetic Resonance Imaging 10(3), 223-232 (1999).

[28] P. S. Tofts and A. G. Kermode. "Measurement of the blood-brain barrier permeability and leakage space using dynamic MR imaging. 1. Fundamental concepts." Magnetic Resonance in Medicine 17(2), 357-367. ISSN 1522-2594 (1991).

[29] G. J. M. Parker, C. Roberts, A. Macdonald, G. A. Buonaccorsi, S. Cheung, D. L. Buckley, A. Jackson, Y. Watson, K. Davies, and G. C. Jayson. "Experimentallyderived functional form for a population-averaged high-temporal-resolution arterial input function for dynamic contrast-enhanced MRI." Magnetic Resonance in Medicine 56(5), 993-1000. ISSN 0740-3194 (2006).

[30] C. Foottit, G. O. Cron, M. J. Hogan, T. B. Nguyen, and I. G. Cameron. "Determination of the Venous Output Function From MR Signal Phase: Feasibility 
for Quantitative DCE-MRI in Human Brain." Magnetic Resonance in Medicine 63(3), 772-81. ISSN 1522-2594 (2010).

[31] A. Garpebring, R. Wirestam, J. Yu, T. Asklund, and M. Karlsson. "Phase-based arterial input functions in humans applied to dynamic contrast-enhanced MRI: potential usefulness and limitations." Magnetic Resonance Materials in Physics, Biology, and Medicine 24(4), 233-245 (2011).

[32] A. Haase, J. Frahm, D. Matthaei, W. Hänicke, and K. D. Merboldt. "FLASH imaging: rapid NMR imaging using low flip-angle pulses." Journal of Magnetic Resonance 67(2), 258-266. ISSN 1096-0856 (1986).

[33] W. Hanicke, K. Merboldt, D. Chien, M. Gyngell, H. Bruhn, and J. Frahm. "Signal strength in subsecond FLASH magnetic resonance imaging: The dynamic approach to steady state." Medical Physics 17(6), 1004-1010 (1990).

[34] G. O. Cron, G. Santyr, and F. Kelcz. "Accurate and rapid quantitative dynamic contrast-enhanced breast MR imaging using spoiled gradient-recalled echoes and bookend T1 measurements." Magnetic Resonance in Medicine 42(4), 746-53. ISSN 0740-3194 (1999).

[35] P. A. Gowland and M. O. Leach. "Fast and Accurate Measurements of T1 Using a Multi-readout Single Inversion-Recovery Sequence." Magnetic Resonance in Medicine 26(1), 79-88 (1992).

[36] C. Boetes, J. Barentsz, R. Mus, R. van der Sluis, L. van Erning, J. Hendricks, R. Holland, and S. Ruys. "MR Characterization of suspicious breast lesions with a gadolinium-enhanced TurboFLASH subtraction technique." Radiology 193(3), 777-781 (1994).

[37] H. Y. Carr. "Steady-State Free Precession in Nuclear Magnetic Resonance." Physical Review 112(5), 1693-1701 (1958).

[38] J. Frahm, W. Hanicke, and K. D. Merboldt. "Transverse Coherence in Rapid FLASH NMR Imaging." Journal of Magnetic Resonance 72(2), 307-314. ISSN 00222364 (1987).

[39] A. P. Crawley, M. L. Wood, and R. M. Henkelman. "Elimination of Transverse Coherences in FLASH MRI." Magnetic Resonance in Medicine 8(3), 248-60. ISSN 0740-3194 (1988).

[40] E. Hahn. "An Accurate Nuclear Magnetic Resonance Method for Measuring Spin-Lattice Relaxation Times." Physical Review 76(1), 145-146 (1949). 
[41] A. Ejchart, P. Oleski, and K. Wroblewski. "Extended Inversion-Recovery Method for Spin-Lattice Relaxation Measurements . A Key to Accurate T1 Determination." Journal of Magnetic Resonance 68(2), 207-216 (1986).

[42] S. G. Kim, X. Hu, and K. Uurbil. "Accurate T1 determination from inversion recovery images: application to human brain at 4 Tesla." Magnetic Resonance in Medicine 31(4), 445-9. ISSN 0740-3194 (1994).

[43] R. K. Gupta, J. A. Ferretti, E. D. Becker, and G. H. Weiss. "A modified fast inversion-recovery technique for spin-lattice relaxation measurements." Journal of Magnetic Resonance 38(3), 447-452. ISSN 00222364 (1980).

[44] H. Z. Wang, S. J. Riederer, and J. N. Lee. "Optimizing the precision in T1 relaxation estimation using limited flip angles." Magnetic Resonance in Medicine 5(5), 399-416. ISSN 0740-3194 (1987).

[45] E. K. Fram, R. J. Herfkens, G. A. Johnson, G. H. Glover, J. P. Karis, A. Shimakawa, T. G. Perkins, and N. J. Pact. "Rapid Calculation of T1 Using Varaible Flip Angle Gradient Refocused Imaging." Magnetic Resonance Imaging 5(3), 201-208 (1987).

[46] J. Homer and M. S. Reevers. "Driven-Equilibrium Single-Pulse Observation of T1 A Reevaluation of a Rapid New Method for Determining NMR Spin-Lattice Relaxation Times." Journal of Magnetic Resonance 63(2), 287-297 (1985).

[47] J. A. Brookes, T. W. Redpath, F. J. Gilbert, A. D. Murray, and R. T. Staff. "Accuracy of T1 measurement in dynamic contrast-enhanced breast MRI using two- and three-dimensional variable flip angle fast low-angle shot." Journal of Magnetic Resonance Imaging 9(2), 163-71. ISSN 1053-1807 (1999).

[48] B. R. Condon, J. Patterson, D. Wyper, A. Jenkins, D. M. Hadley, and M. R. Unit. "Image non-uniformity in magnetic resonance imaging: its magnitude and methods for its correction." The British Journal of Radiology 60(January), 83-87 (1987).

[49] G. J. Barker, A. Simmons, S. R. Arridge, and P. S. Tofts. "A simple method for investigating the effects of non-uniformity of radiofrequency transmission and radiofrequency reception in MRI." The British Journal of Radiology 71(841), 59-67. ISSN 0007-1285 (1998).

[50] L. I. Sacolick, F. Wiesinger, I. Hancu, and M. W. Vogel. "B1 mapping by BlochSiegert shift." Magnetic Resonance in Medicine 63(5), 1315-22. ISSN 1522-2594 (2010). 
[51] V. L. Yarnykh. "Actual flip-angle imaging in the pulsed steady state: a method for rapid three-dimensional mapping of the transmitted radiofrequency field." Magnetic Resonance in Medicine 57(1), 192-200. ISSN 0740-3194 (2007).

[52] G. J. Parker, G. J. Barker, and P. S. Tofts. "Accurate multislice gradient echo T1 measurement in the presence of non-ideal RF pulse shape and RF field nonuniformity." Magnetic Resonance in Medicine 45(5), 838-45. ISSN 0740-3194 (2001).

[53] J. Wang, M. Qiu, H. Kim, and R. T. Constable. "T1 measurements incorporating flip angle calibration and correction in vivo." Journal of Magnetic Resonance 182(2), 283-92. ISSN 1090-7807 (2006).

[54] S. C. L. Deoni. "High-resolution T1 mapping of the brain at 3T with driven equilibrium single pulse observation of $\mathrm{T} 1$ with high-speed incorporation of RF field inhomogeneities (DESPOT1-HIFI)." Journal of Magnetic Resonance Imaging 26(4), 1106-11. ISSN 1053-1807 (2007).

[55] S. Akoka, F. Franconi, F. Seguin, and A. Le Pape. "Radiofrequency map of an NMR coil by imaging." Magnetic Resonance Imaging 11(3), 437-441 (1993).

[56] R. Graumann, A. Oppelt, and E. Stetter. "Multiple-spin-echo imaging with a 2D Fourier method." Magnetic Resonance in Medicine 3(5), 707-21. ISSN 0740-3194 (1986).

[57] D. Burstein. "Stimulated Echoes: Practical Hints." Concepts in Magnetic Resonance 8(4), 269-278 (1996).

[58] D. C. Look and D. Locker. "Time Saving in Measurement of NMR and EPR Relaxation Times." Review of Scientific Instruments 41(2), 250. ISSN 00346748 (1970).

[59] A. P. Crawley and R. M. Henkelman. "A comparison of one-shot and recovery methods in T1 imaging." Magnetic Resonance in Medicine 7(1), 23-34. ISSN 0740-3194 (1988).

[60] Y. Zhang, H. Yeung, P. Carson, and J. Ellis. "Experimental Analysis of T1 imaging with a Single-Scan, Multiple-Point, Inversion-Recovery Technique." Magnetic Resonance in Medicine 25(1), 337-343 (1992).

[61] R. Deichmann and A. Haase. "Quantification of Tl Values by SNAPSHOTFLASH NMR Imaging." Journal of Magnetic Resonance 96(3), 608-612 (1992). 
[62] C. Y. Tong and F. S. Prato. "A novel fast T1-mapping method." Journal of Magnetic Resonance Imaging 4(5), 701-8. ISSN 1053-1807 (1994).

[63] D. R. Messroghli, A. Radjenovic, S. Kozerke, D. M. Higgins, M. U. Sivananthan, and J. P. Ridgway. "Modified Look-Locker inversion recovery (MOLLI) for highresolution T1 mapping of the heart." Magnetic Resonance in Medicine 52(1), 141-6. ISSN 0740-3194 (2004).

[64] A. Oppelt, R. Graumann, H. Barfuss, H. Fisher, W. Hartl, and W. Schjor. "FISP-a new fast MRI sequence." Electromedica 54(1), 15-18 (1986).

[65] J. P. Wansapura, S. K. Holland, R. S. Dunn, and W. S. Ball. "NMR relaxation times in the human brain at 3.0 tesla." Journal of Magnetic Resonance Imaging 9(4), 531-8. ISSN 1053-1807 (1999).

[66] P. Wedeking, C. H. Sotak, J. Telser, K. Kumar, C. A. Chang, and M. F. Tweedle. "Quantitative dependence of MR signal intensity on tissue concentration of Gd(HP-DO3A) in the nephrectomized rat." Magnetic Resonance Imaging 10(1), 97-108. ISSN 0730-725X (1992). 